\title{
CHARACTERIZATION OF AMMONIUM POLYURANATE POWDERS FROM A CONTINUOUS PRECIPITATOR
}

Timothy Oolman

Based on a M.S. thesis submitted to Iowa State University

Ames Laboratory, USDOE Iowa State University

Ames, Iowa 50011

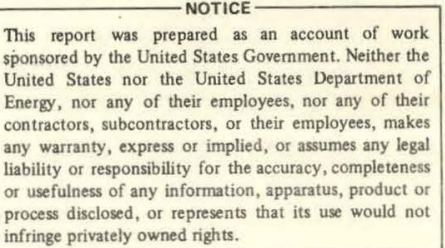

Date Transmitted: January 1979

PREPARED FOR THE U.S. DEPARTMENT OF ENERGY UNDER CONTRACT NO. W-7405-eng-82 


\section{DISCLAIMER}

This report was prepared as an account of work sponsored by an agency of the United States Government. Neither the United States Government nor any agency Thereof, nor any of their employees, makes any warranty, express or implied, or assumes any legal liability or responsibility for the accuracy, completeness, or usefulness of any information, apparatus, product, or process disclosed, or represents that its use would not infringe privately owned rights. Reference herein to any specific commercial product, process, or service by trade name, trademark, manufacturer, or otherwise does not necessarily constitute or imply its endorsement, recommendation, or favoring by the United States Government or any agency thereof. The views and opinions of authors expressed herein do not necessarily state or reflect those of the United States Government or any agency thereof. 


\section{DISCLAIMER}

Portions of this document may be illegible in electronic image products. Images are produced from the best available original document. 


\section{NOTICE}

This report was prepared as an account of work sponsored by the United States Government. Neither the United States nor the United States Departmenit of Energy, nor any of their employees, nor any of their contractors, subcontractors, or their employees, makes any warranty, express or implied, or assumes any legal liability or responsibility for the accuracy, completeness, or usefulness of any information, apparatus, product or process disclosed, or represents that its use would not infringe privately owned rights.

Available from: National Technical Information Service

U. S. Department of Commerce

P.O. Box 1553

Springfield, VA 22161

Price: Microfiche $\$ 3.00$ 


\begin{abstract}
Characterization of ammonium polyuranate powders from a continuous precipitator
\end{abstract}

by

Timothy Oolman

A Thesis Submitted to the

Graduate Faculty in Partial Fulfillment of

The Requirements for the Degree of

MASTER OF SCIENCE

Major: Chemical Engineering

Approved :
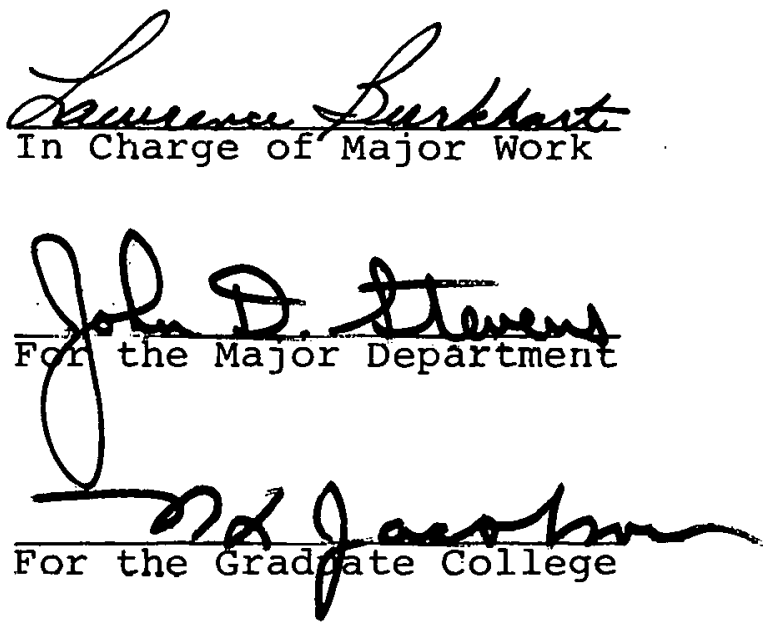

Iowa State University

Ames, Iowa 
TABLE OF CONTENTS

\begin{tabular}{|c|c|}
\hline & PAGE \\
\hline Abstract & viii \\
\hline NOMENCLATURE & \\
\hline INTRODUCTION & 1 \\
\hline LITERATURE REVIEW & 4 \\
\hline THEORY & 11 \\
\hline Crystallite Population Balance & 14 \\
\hline Cluster Population Balance & 16 \\
\hline Agglomerate Population, Balance & 17 \\
\hline Overall Population Balance & 19 \\
\hline EXPERIMENTAL PROCEDURE & 32 \\
\hline DATA ANALYSIS & 38 \\
\hline RESULTS AND DISCUSSION & 42 \\
\hline Characterization of APU Particle Structures & 45 \\
\hline $\begin{array}{l}\text { Correlation of SEM Results with those of the } \\
\text { Coulter Counter }\end{array}$ & 59 \\
\hline TEM Characterization of Crystallites & 61 \\
\hline Calculation of Kinetic Parameters & 79 \\
\hline Phase Analysis & 89 \\
\hline SUMMARY AND CONCLUSIONS & 91 \\
\hline BIBLIOGRAPHY & 94 \\
\hline ACKNOWLEDGMENTS & 98 \\
\hline
\end{tabular}




\section{NOMENCLATURE ${ }^{1}$}

A

$\mathrm{B}^{\mathrm{O}}$

C

G

$\mathrm{G}_{\mathrm{a}}$

$G_{a}^{\prime}$

$G_{C}$

Ge

i

j

k

k

$\mathrm{k}_{\mathrm{c}}$

$\mathrm{k}_{\mathrm{N}}, \mathrm{k}^{\prime}$

$\mathrm{k}_{\mathrm{x}}^{\prime}$

$k_{x}^{\prime \prime}$

L

$\overline{\mathrm{I}}$

surface area per unit volume of suspension, $L^{2} / L^{3}$ nucleation rate per unit volume of suspension, $\# /\left(L^{3} \cdot t\right)$

solute concentration, $M / L^{3}$

growth rate, $\mathrm{L} / \mathrm{t}$

average agglomerate growth rate resulting from cluster coagulation, $\mathrm{L} / \mathrm{t}$

average agglomerate growth rate resulting from coagulation of crystalites with agglomerates, L/t áverage cluster growth rate resulting from coagulation of crystalites into clusters, $\mathrm{L} / \mathrm{t}$

effective growth rate, $\mathrm{L} / \mathrm{t}$

growth rate kinetic order of nucleation

suspension density kinetic order of nucleation

coagulation constant, $1 / t$

size-independent surface area shape factor

average coagulation constant of crystallites into clusters, $1 / t$

nucleation constants

average coagulation constant of crystallites into clusters, $1 / t$

average coagulation constant of crystallites with agglomerates, $1 / t$

particle size (characteristic dimension), L

average particle size, I

IUnits are represented as; $M=$ mass, $L=$ length, $t=$ time, \# = number, $T=$ temperature. 
m

M

$\mathrm{M}_{\mathrm{F}}$

$\mathrm{n}$

$\bar{n}$

$\mathrm{N}$

$\mathrm{P}$

$Q$

$\mathrm{S}$

t

V

$\mathbf{w}$

mass of particles per unit volume of suspension, $M / L^{3}$

M suspension density per unit volume of suspension, $\mathrm{M} / \mathrm{L}^{3}$

M suspension density fraction

particle populations density, \#/(L.L $\left.{ }^{3}\right)$

average population density in size range $\Delta \mathrm{L}$, $\# /\left(L \cdot L^{3}\right)$

Number of particles per unit volume of suspension, $\# / r^{3}$

solute production rate, $M / t$

total feed flow rate, $L^{3} / t$

degree of supersaturation, $M / L^{3}$

time, $t$

suspension holdup volume in precipitator, $\mathrm{L}^{3}$

mass fraction of particles per unit volume of suspension

Greek symbols

$\rho \quad$ particle density, $M / L^{3}$

$\tau$ residence time, $t$

Superscripts

- particles of nucleate size 


\section{Subscripts}
a
agg lomerates
C
clusters
d
dominant size
m
minimum size
$\circ$
initial conditions
$\mathrm{T}$
crystallites + clusters + agglomerates
$\mathbf{x}$
crystallites
1
conditions that exist for the particles at the lower edge of the size range $\Delta \mathrm{L}$
conditions that exist for the particles at the higher edge of the size range $\Delta L$

2 
Abstract

Ammonium polyuranate powders produced in a continuous, well-mixed precipitator were characterized by means of electron microscopy. The powders were qualitatively analyzed with the scanning electron microscope and the elementary crystallites were quantitatively analyzed with the transmission electron microscope. The results were fit to a kinetic theory of continuous precipitation. A phase analysis was also preformed by $x$-ray powder diffraction. 


\section{INTRODUCTION}

A large number of amorphous inorganic materials can be produced by way of the powder sintering process to give a product with properties not attainable by more conventional means. The term "sintering" is used to describe the phenomena which occur when useful solid products are made from inorganic powders -- either metallic or nonmetallic. The process is accomplished relatively easily. An array of particles of suitable size (commonly a few microns or less in diameter) is heated to a temperature between $1 / 2$ and $3 / 4$ of the absolute melting point for times on the order of one hour. During this treatment the particles join together, the piece shrinks, and much of the void volume which results from the initial misfit of the powder particles is eliminated.

The sintering of nonmetallic powders produces brittle ceramic products which cannot be plastically deformed or heat treated to control the final product as a sintered metal product could be. Since the microstructure present at the end of the sintering operation is that which appears in the final product, ceramists must focus the most attention on the development of porosity and grain structure during the sintering process. Of particular importance in this respect is the state of subdivision of the powder before the 
sintering process, as reflected by particle sizes, size distribution, particle morphology, and the extend of agglomeration.

A promising application of the sintering process is the production of polycrystalline ceramics for use in visible and infrared optical systems (4). The ultratransparent $\mathrm{ThO}_{2}$-doped $\mathrm{Y}_{2} \mathrm{O}_{3}$ ceramic is one such example. The desirability of producing large lenses with good optical quality and high strength makes the powder sintering process the only practical method of fabrication. The primary problem with sintered polycrystalline optical equipment is the existence of residual porosity which acts as light scattering centers. Therefore the production of optical ceramics completely free from pores is the primary goal of the ceramist.

The present work deals in general with a second important application of the sintering process -- the fabrication of $\mathrm{UO}_{2}$ fuel pellets by precipitation and subsequent calcination, reduction, and sintering of the ammonium polyranate (APU) powder. The microstructure of the final $\mathrm{UO}_{2}$ ceramic product (density, porosity, grain size, and pore size distribution) are determining factors in the in-core performance of the fuel $(7,26)$. Therefore, the optimization of the microstructure of the pellet is essential for producing the most desirable product. As in the case of polycrystalline ceramic optical systems, there is general agreement among the people working in the area of fuel fabrication that the 
properties of the $\mathrm{UO}_{2}$ ceramic product depend upon both the properties of the original powder and the pellet fabrication technique. Each step of the fabrication process, including precipitation, calcination, milling, granulation, pressing, sintering, and grinding, can have, a significant effect on the properties of the product. However, only the initial precipitation of the APU powder will be considered in the present work.

The ammonium polyuranate powders which are discussed in this work were produced by continuous precipitation from uranyl nitrate solutions to which ammonium hydroxide was added. This precipitation process involves the nucleation and growth of individual crystallites, the formation of clusters of these crystallites, and the agglomeration of the clusters. Adequate characterization of the uranate powders must distinguish between these three types of particles and must describe the morphology and size distribution of each. 


\section{LITERATURE REVIEW}

The most widely used method for the commercial production of $\mathrm{UO}_{2}$ powders is the calcination and reduction of ammonium polyuranate (APU) which is precipitated from uranyl nitrate solution by the addition of ammonia $(7,8,15)$. This method produces a $\mathrm{UO}_{2}$ powder of greater reactivity and higher density than that prepared by other methods. In some cases the fuel production is even more economical because the $\mathrm{UO}_{2}$ powder can be cold-pressed and subsequently sintered without the addition of a binder (28).

The APU precipitate is often referred to as ammonium diuranate; however this compound has never been isolated from solution and the exact composition is still in question. The complexity and uncertainty of the reactions involved is indicated by the large number of chemical systems which have been postulated for the reaction of uranyl nitrate and ammonium hydroxide by various authors $(13,24,30)$. All of the studies have indicated that equilibrium is established slowly and is affected by the addition rate of the reactants.

Cordfunke (9) and Debets and Loopstra (12) have reported that in equilibrium with aqueous solutions, four well-defined compounds exist in the $\mathrm{NH}_{3}-\mathrm{UO}_{3}-\mathrm{H}_{2} \mathrm{O}$ system: 


$$
\begin{array}{ll}
\text { I } & \mathrm{UO}_{3} \cdot 2 \mathrm{H}_{2} \mathrm{O} \\
\text { II } & 3 \mathrm{UO}_{3} \cdot \mathrm{NH}_{3} \cdot 5 \mathrm{H}_{2} \mathrm{O} \\
\text { III } & 2 \mathrm{UO}_{3} \cdot \mathrm{NH}_{3} \cdot 3 \mathrm{H}_{2} \mathrm{O} \\
\text { IV } & 3 \mathrm{UO}_{3} \cdot 2 \mathrm{NH}_{3} \cdot 4 \mathrm{H}_{2} \mathrm{O}
\end{array}
$$

where compound II is formed at a pH of 4-7 and compounds III and IV seem to prevail above a $\mathrm{pH}$ of 7 . However, isolation of the pure form of any of these compounds cannot easily be done due to the presence of nitrate, which cannot be removed completely by washing with water. Debets and Loopstra have reported the X-ray powder patterns for all four compounds and the crystal structures obtained therefrom. It was observed that compounds III and IV are hygroscopic. Upon prolonged standing in moist air part of the ammonia in the product is exchanged for water. This can cause a change of the positions of the $\mathrm{x}$-ray reflections of over 0.5 degrees.

Due to the range and uncertainty of its composition, the precipitates studied in this work will.be generally referred to as ammonium polyuranate (APU) with no specific composition implied.

Previous APU precipitation studies $(14,19)$ have shown the process to include the nucleation and growth of thin, elementary platelets $(0.025$ to $0.5 \mu \mathrm{m})$ which strongly bond into small clusters $(0.5$ to $3 \mu \mathrm{m})$, that in turn may loosely 
agglomerate into still larger particles (2 to $30 \mu \mathrm{m}$ ). Hoyt (18) was the first to combine these three processes into a continuous precipitation model. The model is based on three dynamic population balances which are derived for the crystallites, clusters, and agglomerates, respectively, with interpretation of the coagulation processes by average coagulation growth rates. The steady state solutions of these population balances result in expressions for the size distributions, average particle sizes, dominant particle sizes, and suspension densities of the crystallites, clusters, and agglomerates leaving the precipitator. Hoyt then applied this kinetic model to the continuous precipitation of APU. He concluded that the particle size distribution and particle structure of the APU precipitate can be controlled through proper regulation of the precipitation conditions -- in particular the uranium concentration, the $\mathrm{NH}_{3}: \mathrm{U}$ reactor feed mole ratio, and the residence time in the precipitator.

Hoyt also developed titration curves of $\mathrm{pH}$ vs. $\mathrm{NH}_{3}: \mathrm{U}$ reacting feed mole ratio for varying uranium concentrations, as given in Figure 1. His experimental study of the APU precipitation process included characterization of the particle size distribution of the clusters and agglomerates by use of a model TA-II Coulter counter for which the small size detection limit is a volume equivalent diameter of 


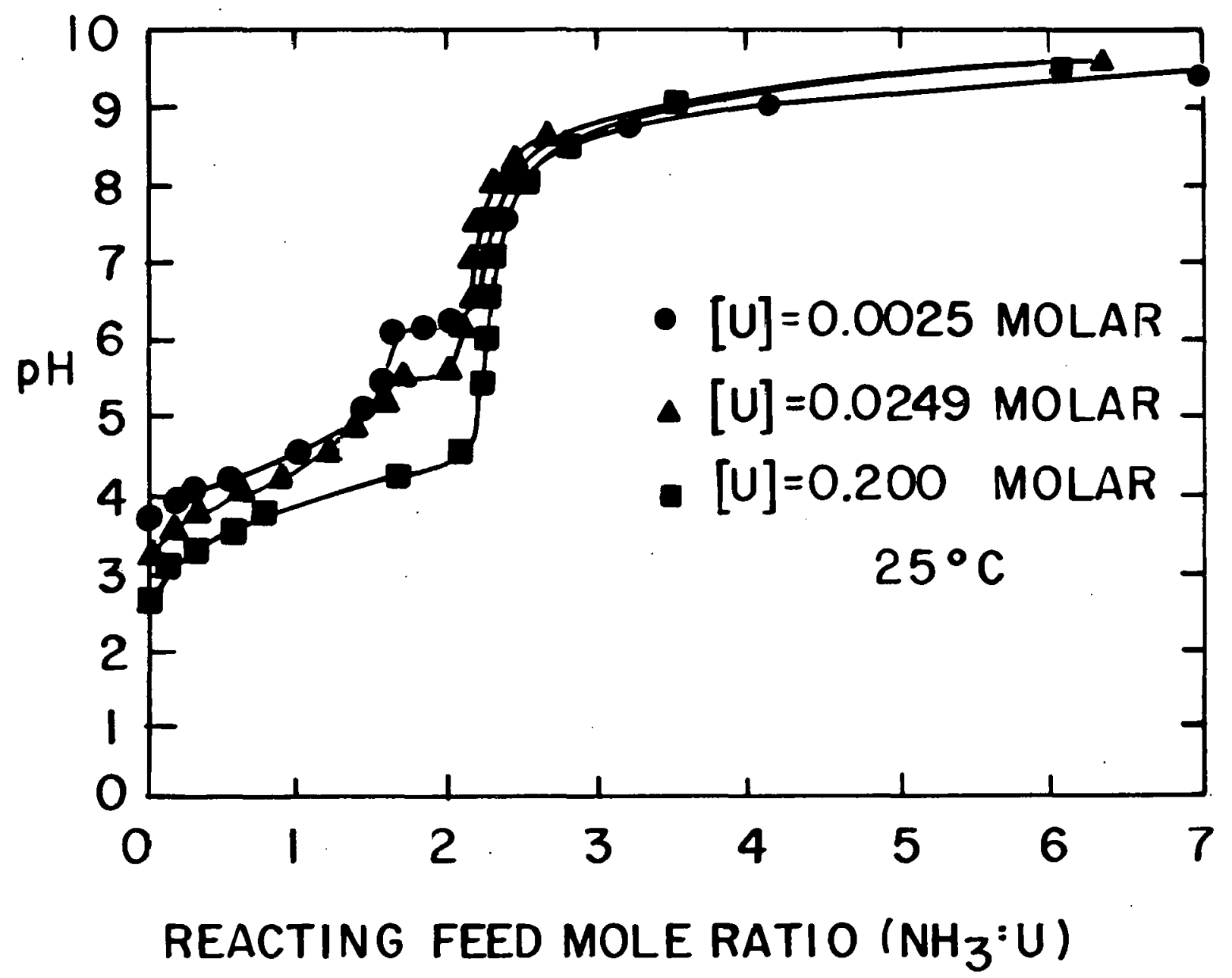

Figure 1. Titration curves for different uranium concentrations (ammonium hydroxide being acided to uranyl nitrate solution). 
about a half micron. Since in all cases the average crystallite size of the APU powder is far below this limit, this method of analysis was inadequate for the measurement of the crystallite particle size distributions. Therefore, values of the crystallite growth rates, average crystallite sizes, and a number of related parameters were not obtained. It is the purpose of the present study to fill in this missing information by using electron microscopy to study the crystallites.

Behringer, et al. (5), correlated electron microscope results with those of the coulter counter and found the Coulter counter measurements of a polylatex material to be consistently about $0.2 \mu \mathrm{m}$ higher. Excellent agreement was obtained, however, for the measurement of a polystyrene material and NBS 1003 calibrated glass beads.

Electron microscopy can be conveniently divided into two fields. The traditional field of transmission electron microscopy, which produces an 1mage with a resolution limit of 5-10 $\AA$ by transmitting an electron beam through the sample, was first introduced commercially in 1939. The much more recently introduced scanning electron microscope, which became commercially available in 1964, produces an image with a maximum resolution of about $100 \AA$ by detecting secondary (low energy) electrons emitted from the surface of the sample. This is in comparison to a resolution 
limit of about $0.2 \mu \mathrm{m}(2000 \AA)$ for the best optical microscopes.

The rapid growth in application of the scanning electron microscope (SEM) in the past decade has been due to its complementary rather than competitive function in relation to the transmission electron microscope (TEM). Since the SEM relies on a reflected rather than transmitted electron beam it provides a means for studying the surface characteristics of a material which is much too thick to study directly with the TEM, and yields a resolution and depth of focus far beyond the limits of an optical microscope. These complementary functions of the SEM and TEM make them an invaluable pair of tools for studying the characteristics of APU precipitates. The SEM provides a means for studying the surface characteristics of the clusters and agglomerates as well as providing a correlation with the previously reported coulter counter results. The TEM provides a method for studying the crystallite size distribution and morphology.

Several excellent texts and papers are available on the theory and operation of, as well as sample preparation for, both the SEM $(17,21)$ and the TEM $(3,32)$. White (31) has summarized several applications of the SEM to ceramic studies, including a study of the kinetics of crystallization. Johari and Bhattacharyya (20) examined 12 metal and alloy powders with the SEM and concluded that the SEM should be 
very useful in the field of powder studies in providing information pertaining to size, shape, surface topography and texture, porosity and pore shape, microstructure and agglomeration tendencies. Crowl (10) conducted a particle size analysis of a titanium dioxide pigment by electron microscopy and reported the reproducibility of the sizing and counting operation to be high, with standard deviation generally below 2 per cent. The main source of error was found to be in sample preparation and selection of fields in the electron microscope.

$\mathrm{x}$-ray diffraction is also a common method for powder study. This technique had its beginnings in 1912 when it was discovered that crystals diffract $x$-rays, the manner of the diffraction revealing the structure of the crystal. Today the method is applied to many diverse:problems, such as chemical analysis, measurement of particle sizes, and crystallite orientation within polycrystalline aggregates. In this study $\mathrm{X}$-ray diffraction will be used in correlating phase equilibria with reacting conditions. For the reader who has no previous knowledge of the subject, Cullity (11) presents a useful introduction to the theory of $x$-ray diffraction, the experimental methods involved, and the primary applications thereof. 
THEORY

Precipitation may be considered as a special case of crystallization, where not only a phase change takes place but also a chemical reaction occurs. Crystallization usually involves the phase change of a fairly soluble solute from a concentrated solution at a relatively low level of supersaturation. This situation favors crystal growth rather than nucleation, yielding relatively few large crystals. Precipitation, on the other hand, involves the salting out of a sparingly soluble precipitate at a high level of supersaturation, due to the large production rate involved. This situation favors high nucleation rates rather than crystal growth, yielding a very large number of very small crystallites which may coagulate into larger particles.

The theory of particle size distributions from a continuous mixed-suspension, mixed-produce-renoval (MSMPR) precipitator to be presented in this section is based upon the theory of crystal size distributions from MSMPR crystallizers, which has been thoroughly examined by Randolph and Larson (25). The general development of the kinetic model of continuous precipitation follows the assumption that three processes may take place simultaneously: (1) crystal nucleation and growth, (2) primary coagulation of crystallites to clusters, and (3) secondary coagulation 
of clusters to agglomerates. All three of these processes have been reported in the literature to occur during precipitation. The combination of these into a continuous precipitation model with interpretation of the coagulation processes by average coagulation growth rates, as is developed subsequently, was first presented by Hoyt. (18).

Assuming that the particles can be described by some characteristic dimension one can define size-invariant surface area and volumetric shape factors, $\mathrm{k}_{\mathrm{a}}$ and $\mathrm{k} \mathrm{v}$ respectively.' The product of the square of the characteristic dimension with the surface area shape factor determines the surface area of the particle, and the product of the cube of the characteristic dimension with the volumetric shape factor gives the volume of the particle. Since the APU crystallites are thin platelets the characteristic dimension used to describe them will be the area equivalent diameter of the face of the platelet, i.e., the diameter of a circle of the same area as one face of the platelet. On the other hand Hoyt's work describes the clusters and agglomerates in the system by using a characteristic dimension of the volume equivalent diameter, i.e., the diameter of a sphere of the same volume as the particle being considered. As a result, the surface area and volumetic shape factors of the crystallites will differ from those of the clusters and agglomerates. 
The development of the precipitation model involves writing a population balance equation for each of the three types of particles with the following assumptions being made :

1) no particles in the feed solutions

2) perfect mixing

3) no product withdrawal classification

4) negligible particle breakage and attrition

5) uniform shape factors

6) constant suspension holdup volume

The population density of any type of particle can be defined as

$$
\operatorname{limit}_{\Delta \mathrm{L} \rightarrow 0} \frac{\Delta \mathrm{N}^{\prime}}{\Delta \mathrm{L}}=\frac{d \mathrm{~N}^{\prime}}{\mathrm{dL}}=\mathrm{n}(\mathrm{L})
$$

where $N^{\prime}$ is the number of particles in the size range $\Delta \mathrm{L},\left(\mathrm{L}_{1}\right.$ to $\mathrm{L}_{2}$ ), per unit volume of suspension. The total number of particles of any one.type in this size range is then given by

$$
\Delta N^{\prime}=\int_{L_{1}}^{L_{2}}(L) d L
$$

and the total number of particles of any one type in all size ranges is then given by 


$$
N_{T}^{\prime}=\int_{0}^{\infty} n(L) d L
$$

\section{Crystallite Population Balance}

If one considers a constant suspension volume $\mathrm{V}$ and $\mathrm{a}$ time interval $\Delta t$, then the dynamic crystallite population balance (accumulation = input - output) for an arbitrary size range $\mathrm{L}_{1}$ to $\mathrm{L}_{2}$ is.

accumulation $=$ (crystallites growing into size range $\Delta L)$ - (crystallites in size range $\Delta L$ flowing out of the reactor) - (crystallites growing out of size range $\Delta L$ ) - (crystallites in size range $\Delta L$ which coagulate into clusters) - (crystallites in size $\Delta L$ which coagulate with agglomerates).

This can be written as

$$
\begin{aligned}
V \Delta n_{x} \Delta L= & \left(n_{1, x} G_{1, x} \Delta t V\right)-\left(Q \bar{n}_{x} \Delta t \Delta L\right)-\left(n_{2}, x_{2}, x+t V\right)- \\
& \left(\bar{n}_{x}{ }_{x}^{\prime} \Delta t \Delta L V\right)-\left(\bar{n}_{x}{ }_{x}^{\prime \prime}{ }_{x} \Delta t \Delta L V\right)
\end{aligned}
$$

where the subscripts 1 and 2 refer to conditions that exist for the particles at the lower and higher edge of the size range $\Delta \mathrm{L}$ respectively, $\mathrm{x}$ refers to the crystallite conditions, $\bar{n}_{x}$ is the average crystallite population density in the size range $\Delta \mathrm{L}, \mathrm{G}$ is the growth rate, $Q$ is the total feed flow rate or output flow rate, $k_{x}^{\prime}$ is the average 
coagulation constant for the crystallites coagulating into clusters, and $k_{x}^{\prime \prime}$ is the average coagulation constant for crystallites coagulating with agglomerates. Rearranging equation (4) and dividing by $V \Delta t \Delta L$ gives

$$
\begin{aligned}
& \frac{\Delta n_{x}}{\Delta t}+\frac{1}{\tau} \bar{n}_{x}+\left(n_{2, x} G_{2, x}-n_{1, x} G_{1, x}\right) / \Delta L+ \\
& \left(k_{x}^{\prime}+k_{x}^{\prime \prime}\right) \bar{n}_{x}=0
\end{aligned}
$$

where $\tau$ is defined as the residence time of the process, $\mathrm{V} / \mathrm{Q}$. Allowing $\Delta \mathrm{L}$ and $\Delta t$ to approach zero yields

$$
\frac{\partial n_{x}}{\partial t}+\frac{\partial}{\partial L}\left(G_{x} n_{x}\right)+\left(\frac{1}{\tau}+k_{x}^{\prime}+k_{x}^{\prime \prime}\right) n_{x}=0
$$

Now if one assumes a size independent growth rate (McCabe's $\Delta \mathrm{L}$ law) then

$$
\frac{\partial n_{x}}{\partial t}+G_{x}\left(\frac{\partial n_{x}}{\partial L}\right)+\left(\frac{1}{\tau}+k_{x}^{\prime}+k_{x}^{\prime \prime}\right) n_{x}=0
$$

Equation (7) represents the dynamic crystallite population balance for the continuous MSMPR precipitator: If steady state is assumed, this reduces to

$$
G_{x}\left(\frac{d n_{x}}{d L}\right)+\left(\frac{1}{\tau}+k_{x}^{\prime}+k_{x}^{\prime \prime}\right) n_{x}=0
$$

the solution for which is

$$
n_{x}=n_{x}^{\circ} \exp \left(-L / \tau G_{e}, x^{)}\right.
$$


where $\mathrm{n}_{\mathrm{x}}^{0}$ is defined as the nucleate density of embryo-size crystals, and $G_{e, x}$ is the effective crystallite growth rate defined as

$$
G_{e, x} \frac{G_{x}}{1+\tau\left(k_{x}^{\prime}+\right.} \frac{\left.k_{x}^{\prime \prime}\right)}{1+x^{2}}
$$

\section{Cluster Population Balance}

A dynamic population balance for the clusters can be derived in a similar manner as that for the crystallites by assuming that the cluster growth due to coagulation can be represented by an average growth rate, $G_{c}$, defined as

$$
G_{C} \equiv k_{x}^{\prime} \bar{L}_{x}
$$

where $\overline{\mathrm{L}}_{\mathrm{x}}$ is the average crystallite size. The steady state dynamic cluster population balance can be written

$$
\left(G_{c}+G_{x}\right) \frac{d n_{c}}{d L}+\left(\frac{1}{\tau}+k_{c}\right) n_{c}=0
$$

where $G_{C}$ and $G_{x}$ are assumed to be size independent and $k_{C}$ is the average coagulation constant of clusters into agglomerates. The solution of Equation (12) is

$$
n_{c}=n_{c}^{O} \exp \left(-L / \tau G_{e, c}\right)
$$


where $\mathrm{n}_{\mathrm{x}}^{0}$ is the extrapolated cluster nucleate population density and $G_{e, x}$ is the effective cluster growth rate defined as

$$
G_{e, c} \equiv \frac{G_{c}+G_{x}}{1+k_{c}}
$$

\section{Agglomerate Population Balance}

If one assumes that (I) the agglomerate growth rate as a result of coagulation of clusters can be described as an average coagulation growth rate, $G_{a}$, defined as

$$
G_{a} \equiv k_{c} \bar{L}_{c}
$$

where $\bar{L}_{c}$ is the average cluster size, and (2) that the agglomerate growth rate as a result of the coagulation of crystallites with agglomerates can be described as an average coagulation growth rate, $G_{a}^{\prime}$, defined as

$$
G_{a}^{\prime} \equiv k_{x}^{\prime \prime} \bar{L}_{x}
$$

then the dynamic agglomerate population balance can be written for steady state reacting conditions as

$$
\left(G_{a}+G_{a}^{\prime}+G_{x}\right) \frac{d n_{a}}{d I}+\frac{1}{\tau} n_{a}=0
$$

where $G_{a}, G_{a}^{\prime}$ and $G_{x}$ are assumed to be size independent. The solution for Equation 17 is 


$$
n_{a}=n_{a}^{O} \exp \left(-L / \tau G_{e, a}\right)
$$

where $\mathrm{n}_{\mathrm{a}}^{\mathrm{O}}$ is the extrapolated agglomerate nucleate population density, and $G_{e, a}$ is the effective agglomerate growth rate defined as

$$
G_{e, a}=G_{a}+G_{a}^{\prime}+G_{x}
$$

The steady state solutions describing the population density distributions of clusters and agglomerates (Equations (13) and (18)) assume that the population densities are continuous in a size range from 0 to $\infty$; however in reality the average minimum size of the clusters and agglomerates will not be zero. Since the volume of the smallest cluster must be equivalent to the volume of two crystallites, the average minimum cluster size, $\overrightarrow{\mathrm{L}}_{\mathrm{m}, \mathrm{c}^{\prime}}$ will depend on the average crystallite size as given by

$$
k_{v, c} \bar{L}_{m, c}^{3}=2 k_{v, x} \bar{L}_{x}^{3}
$$

or

$$
\overrightarrow{\mathrm{L}}_{\mathrm{m}, \mathrm{c}}=\left(2 \mathrm{k}_{\mathrm{v}, \mathrm{x}} / \mathrm{k}_{\mathrm{v}, \mathrm{c}}\right)^{1 / 3} \overline{\mathrm{L}}_{\mathrm{x}}
$$

where $\overline{\mathrm{L}}_{x}$ is the average crystallite size calculated by

$$
\bar{L}_{x}=\frac{\int_{0}^{\infty} L n_{x}^{\circ} \exp \left(-L / \tau G_{e, x}\right) d L}{\int_{0}^{\infty} n_{x}^{\circ} \exp \left(-L / \tau G_{e, x}\right) d L}=\tau G_{e, x}
$$


Similarly, the average minimum size of the agglomerates will depend upon the average cluster size, the average crystallite size, and upon the coagulation ratios of the crystallites with agglomerates and clusters to agglomerates.

$$
\left.\overline{\mathrm{L}}_{\mathrm{m}, \mathrm{a}}=\left[2\left(\frac{\mathrm{k} v, \mathrm{c}}{\mathrm{k}_{\mathrm{v}, \mathrm{a}}}\right) \overline{\mathrm{L}}_{\mathrm{c}}^{3}+\left(\frac{\mathrm{k}_{\mathrm{x}}^{\prime \prime}}{\mathrm{k}_{\mathrm{c}}}\right)\left(\frac{\mathrm{k}, \mathrm{v}, \mathrm{x}}{\mathrm{k}, \mathrm{v}, \mathrm{a}}\right) \overline{\mathrm{L}}_{\mathrm{x}}^{-3}\right]\right]^{1 / 3}
$$

where the average size of the clusters, $\overline{\mathrm{L}}_{\mathbf{C}^{\prime}}$ is calculated by

$$
\begin{aligned}
& \overline{\mathrm{L}}_{c}= \frac{\int_{\mathrm{L}, c}^{\infty} \overline{\mathrm{Ln}}_{c}^{\circ} \exp \left(-\mathrm{L} / \mathrm{G}_{e, c}\right) d L}{\int^{\infty} \mathrm{n}_{c}^{\circ} \exp \left(-L / \tau G_{e, c}\right) d L}=\tau G_{e, c}+\bar{L}_{m, c} \\
& \overline{\mathrm{L}}_{\mathrm{m}, c}
\end{aligned}
$$

The average nucleate population density, $\vec{n}^{\circ}$, for clusters and agglomerates can be calculated by evaluating Equations (13) and (18) at the respective average minimum particle sizes.

$$
\begin{aligned}
& \bar{n}_{c}^{\circ}=n_{c}^{\circ} \exp \left(-\bar{L}_{m, c} / \tau G_{e, c}\right) \\
& \overline{\mathrm{n}}_{\mathrm{c}}^{0}=\mathrm{n}_{\mathrm{a}}^{\circ} \exp \left(-\overline{\mathrm{L}}_{\mathrm{m}, \mathrm{a}} / \tau \mathrm{G}_{\mathrm{e}, \mathrm{a}}\right)
\end{aligned}
$$

Overall Population Balance

The total particle population distribution, $\mathrm{n}_{\mathrm{T}}(\mathrm{L})$, leaving the steady state MSMPR precipitator is the sum. of the crystallites, clusters, and agglomerates as given by

$$
\mathrm{n}_{\mathrm{T}}(\mathrm{L})=\mathrm{n}_{\mathbf{x}}(\mathrm{L})+\mathrm{n}_{\mathrm{c}}(\mathrm{L})+\mathrm{n}_{\mathbf{a}}(\mathrm{L})
$$


or

$$
\begin{aligned}
n_{T}(L)= & n_{x}^{\circ} \exp \left(-L / \tau G_{e, x}\right)+n_{c}^{\circ} \exp \left(-L / \tau G_{e, c}\right) \\
& +n_{a}^{\circ} \exp \left(-L / \tau G_{e, a}\right)
\end{aligned}
$$

where it is understood that the clusters and agglomerates do not exist at a size below their respective minimum sizes, $\overline{\mathrm{L}}_{\mathrm{m}, \mathrm{c}}$ and $\overline{\mathrm{L}}_{\mathrm{m}, \mathrm{a}}$.

Equation (27) indicates that if the total particle population distribution from a continuous MSMPR precipitator operating at steady state is plotted on semi-log paper as a function of particle size a curve representing the sum of three exponentials will be obtained. If the effective growth rates are significantly different from one another this curve can be resolved into three straight lines. Each line will represent one of the particle distributions with an intercept equal to $\log n^{\circ}$ and a slope equal to $-1 /(2.303 \tau G)$.

The total number of particles present per unit volume of suspension, $\mathrm{N}_{\mathrm{T}^{\prime}}$ is the sum, over all particle sizes, of the crystallites, clusters, and agglomerates.

$$
\mathrm{N}_{\mathrm{T}}=\mathrm{N}_{\mathrm{x}}+\mathrm{N}_{\mathrm{C}}+\mathrm{N}_{\mathbf{a}}
$$

where 


$$
\begin{aligned}
& N_{x}=\int_{0}^{\infty} n_{x}^{\circ} \exp \left(-L / \tau G_{e, x}\right) d L=n_{x}^{\circ} \tau G_{e, x} \\
& \infty \\
& N_{c}=\int n_{c}^{0} \exp \left(-L / \tau G_{e, c}\right) d L=\bar{n}_{c}^{\circ} \tau G_{e, c} \\
& \overline{\mathrm{L}}_{\mathrm{m}, \mathrm{c}} \\
& \infty \\
& N_{a}=\int n_{a}^{\circ} \exp \left(-L / \tau G_{e, a}\right) d L=\widetilde{n}_{a}^{\circ} \tau G_{e, a} \\
& \overline{\mathrm{L}}_{\mathrm{m}, \mathrm{a}}
\end{aligned}
$$

The average particle sizes for the crystallites and clusters were determined previously and are given in Equations (21) and (23) respectively. The average particle size for the agglomerates can be determined in a similar fashion to be

$$
\overline{\mathrm{L}}_{\mathrm{a}}=\tau \mathrm{G}_{e, a}+\overline{\mathrm{L}}_{\mathrm{m}, \mathrm{a}}
$$

In addition to the steady state population density and average particle size, other characteristics of the different types of particles which are of interest are their respective fractions of the total suspension density and respective dominant particle sizes (that particle size associated with the largest weight fraction). The suspension density, $M$, for any given type of particle can be calculated as

$$
\begin{gathered}
M=k_{v} \rho \int L^{3} n^{\circ} \exp \left(-L / \tau G_{e}\right) d L \\
\left(0 \text { or } \bar{L}_{m}\right)
\end{gathered}
$$


where $\mathrm{k}_{\mathrm{v}}$ is the size-independent volumetric shape factor and $\rho$ is the density of the particle. Application of Equation (33) yields

$$
\begin{aligned}
M_{x}= & 6 \rho_{x} k_{v, x} n_{x}^{\circ}(\tau G e, x)^{4} \\
M_{c}= & \rho_{c} k_{v, c} \bar{n}_{c}^{\circ}\left[\bar{L}_{m, c}^{3} \tau G_{e, c}+3 \bar{L}_{m, c}^{2}\left(\tau G_{e, c}\right)^{2}+\right. \\
& \left.6 \bar{L}_{m, c}\left(\tau G_{e, c}\right)^{3}+6\left(\tau G_{e, c}\right)^{4}\right] \\
M_{a}= & \rho_{a} k_{v, a} \bar{n}_{a}^{\circ}\left[\bar{L}_{m, a}^{3} \tau G_{e, a}+3 \bar{L}_{m, a}^{2}\left(\tau G_{e, a}\right)^{2}+\right. \\
& \left.6 \bar{L}_{m, a}\left(\tau G_{e, a}\right)^{3}+6\left(\tau G_{e, a}\right)^{4}\right]
\end{aligned}
$$

where $\mathrm{M}_{\mathrm{x}}, \mathrm{M}_{\mathrm{C}^{\prime}}$ and $\mathrm{M}_{\mathrm{a}}$ are the crystallite, cluster, and agglomerate suspension densities respectively. The total suspension density is given by

$$
M_{T}=M_{x}+M_{C}+M_{a}
$$

The suspension density fractions for the different types of particles can be calculated as

$$
\begin{aligned}
& M_{F, x}=M_{x} / M_{T} \\
& M_{F, c}=M_{c} / M_{T} \\
& M_{F, a}=M_{a} / M_{T}
\end{aligned}
$$

where $M_{F}$ is the respective suspension density fraction. 
A weight fraction distribution, w(L), can be expressed for a particular type of particle as

$$
w(L)=m(L) / M_{T}
$$

where

$$
m(L)=\rho k_{v} L^{3} n(L)
$$

is the differential mass fraction at size $L$. The respective terms for the crystallites, clusters, and agglomerates can be substituted into Equation (40), yielding three equations which can be differentiated, set equal to zero, and solved for their respective dominant particle sizes. The results of such a procedure are

$$
\begin{aligned}
& \mathrm{L}_{\mathrm{d}, \mathrm{x}}=3 \tau \mathrm{G}_{\mathrm{e}, \mathrm{x}} \\
& \mathrm{L}_{\mathrm{d}, \mathrm{c}}=3 \tau \mathrm{G}_{e, c}+\overline{\mathrm{L}}_{\mathrm{m}, \mathrm{c}} \\
& \mathrm{L}_{\mathrm{d}, \mathrm{a}}=3 \tau \mathrm{G}_{e, a}+\overline{\mathrm{L}}_{\mathrm{m}, \mathrm{a}}
\end{aligned}
$$

where $L_{d}$ is defined as the dominant particle size.

At this point nucleation rates and nucleate population densities will be considered since either one of them, in conjunction with the effective growth rates, will determine the average particle sizes, the dominant particle sizes, and the suspension density fractions. The crystallite nucleation rate, $\mathrm{B}_{\mathrm{x}}^{\circ}$, can be defined as 


$$
B_{x}^{O}=n_{x}^{O} G, x
$$

where the crystallite nucleate population density, $\mathrm{n}_{\mathrm{x}}^{\circ}$, is defined as $\left.\left(\mathrm{dN}_{\mathrm{x}} / \mathrm{dL}\right)\right|_{\mathrm{L}=0}$, and the effective crystal growth rate is defined as dL/dt. From semi-theoretical considerations the following expression can be obtained for the rate of nucleation.

$$
B^{O}=k^{\prime} M^{j} s^{i}
$$

where $M$ is the suspension density, $\mathbf{s}$ is the supersaturation, $k^{\prime}$ is a constant, and $i$ and $j$ are empirically derived kinetic orders of nucleation. In this expression $s^{i}$ represents the effect of homogeneous nucleation and $\mathrm{m}^{\mathrm{j}}$ represents the effect of secondary nucleation. Similarly, crystallite growth can be represented by the expression

$$
\mathrm{dL} / \mathrm{dt} \equiv \mathrm{G}=\mathbf{k}^{*} \mathbf{S}
$$

where $k^{*}$ is a theoretically derived constant. Equations (46), (47), and (48) can be combined to yield

$$
\left.B_{x}^{O}=k_{N, x}\left(G_{e, x}\right)^{i} x_{\left(M_{T}\right.}\right)^{j} x
$$

and

$$
n_{x}^{0}=k_{N, x}\left(G_{e, x}\right)^{i_{x}^{-1}\left(M_{T}\right)} j_{x}^{j}
$$

where $k_{N, x}$ is defined as the crystallite nucleation constant, $i_{x}$ is the crystallite growth rate kinetic order of nucleation, 
and $j_{x}$ is the crystallite suspension density kinetic order of nucleation.

Equation (50) implies that operating the MSMPR precipitator at different levels of supersaturation for the same suspension density will allow the growth rate kinetic order of nucleation, $i$, to be obtained. The level of supersaturation can be experimentally controlled by varying the $\mathrm{NH}_{3}: \mathrm{U}$ reacting feed mole ratio or by varying the residence time in the reactor $(\tau=V / Q)$. By fitting a straight line to a plot of $\log n^{\circ}$ vs. $\log G$ for the experimental data, a value of $i-1$ is obtained from the slope. Operation of the precipitator at different suspension densities will allow determination of the suspension density kinetic order of nucleation, $j$, and the nucleation constant, $k_{N} \cdot$ The suspension density is experimentally controlled by varying the uranium concentration. By fitting a straight line to a plot of $\log \left(n^{\circ} / G_{e}^{i-1}\right)$ vs. $\log M_{T}$, values for $j$ and $k_{N}$ dre obtained from the slope and $y$-intercept respectively. Similarly, nucleate population models for the clusters and agglomerates can be obtained.

$$
\begin{aligned}
& n_{c}^{0}=k_{N, c}\left(G_{e, c}\right)^{i_{c}-1}\left(M_{T}\right) j_{c}^{j} \\
& n_{a}^{0}=k_{N, a}\left(G_{e, a}\right)^{i_{a}-1}\left(M_{T}\right)^{j}
\end{aligned}
$$

It should be noted that equations (51) and (52) are based upon minimum particle sizes being zero. Taking into account 
the average minimum particle sizes, the average nucleate population densities of the clusters and agglomerates will be obtained by combining Equations (24) and (25) with equations (51) and (52).

$$
\begin{aligned}
& \vec{n}_{c}^{o}=k_{N, c}\left(G_{e, c}\right)^{i_{c}-1}\left(M_{T}\right){ }^{j} c_{e x p}\left(-\bar{L}_{m, c} / \tau G_{e, c}\right) \\
& \left.\bar{n}_{a}^{o}=k_{N, a}\left(G_{e, a}^{\cdot}\right)^{i_{a}^{-1}\left(M_{T}\right)}\right)^{j} \exp \left(-L_{m, a} / \tau G e, a\right)
\end{aligned}
$$

Since equations (10), (11), (14), (15), (16), and (19)

form a system of six equations and seven unknowns $\left(G_{x}, G_{C}\right.$ ', $\left.G_{a}, G_{a}^{\prime}, k_{c}, k_{x}^{\prime}, k_{x}^{\prime \prime}\right)$ it is necessary to produce one final relationship which will place a constraint on the crystallite growth rate. If one assumes that the mass rate of phase change due to nucleation and the accumulation of solute as supersaturation are negligible, a mass balance on the solute can be reduced to

$$
G_{x}=2(P / Q-C) / \rho x_{x} \tau A_{T}
$$

where $\mathrm{P}$ is the solute production rate, $\mathrm{C}$ is the solute concentration, $\rho_{X}$ is the crystallite density, and $A_{T}$ is the total particle surface area per unit volume of suspension, defined by

$$
A_{T}=A_{x}+A_{C}+A_{a}
$$

where 


$$
A=k_{a} \int_{\left(0 \text { or } \bar{L}_{m}\right)}^{\infty} n(L) L^{2} d L
$$

for each type of particle. Equation (55) places a suitable constraint on the crystal growth rate in terms of known quantities and allows a model of the continuous MSMPR precipitator to be developed. The informational flow chart for such a model is shown in Figure 2 where it is understood that the various nucleation constants, coagulation constants, and kinetic orders of nucleation are functions of process parameters (reacting feed mole ratio, feed concentrations, suspension density, temperature, agitation, etc.) and can be determined experimentally. Table 1 lists the parameters of the continuous MSMPR precipitation model and shows which ones must be determined experimentally and which ones must be calculated.

The purpose of the present work is to characterize the APU particle structure with the scanning electron microscope and to determine the APU effective crystallite growth rate, $G_{e, x^{\prime}}$ and average crystallite size, $\overline{\mathrm{L}}_{\mathbf{x}}$, with the transmission electron microscope for each of several powder samples.

The work of Hoyt leads to values for the cluster and agglomerate effective growth rates $\left(G_{e, c}\right.$ and $\left.G_{e, a}\right)$, nucleate population densities $\left(n_{c}^{0}\right.$ and $\left.n_{a}^{0}\right)$, kinetic orders of nucleation ( $i_{c}, i_{a}, j_{c}$, and $\left.j_{a}\right)$, nucleation constants $\left(k_{N, c}\right.$ and $\left.k_{N, a}\right)$, 


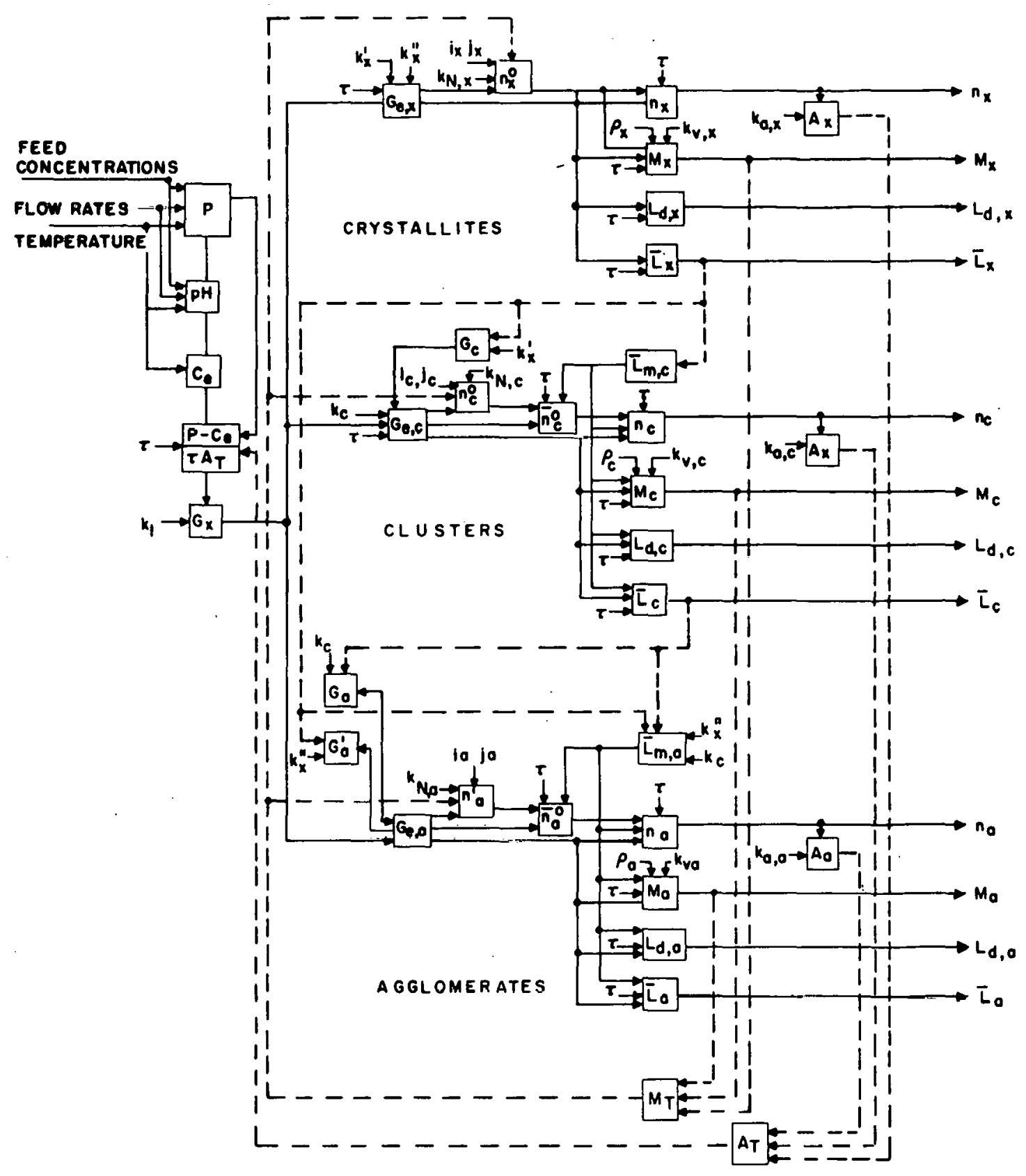

Figure 2. Informational flow diagram for continuous MSMPR precipitation model. 
Table 1. Kinetic parameters and process variables included in the continuous MSMPR precipitation model

Variables determined by precipitation conditions
feed concentrations
feed flow rates
temperature
residence time, $\tau$

Parameters determined from experimental data

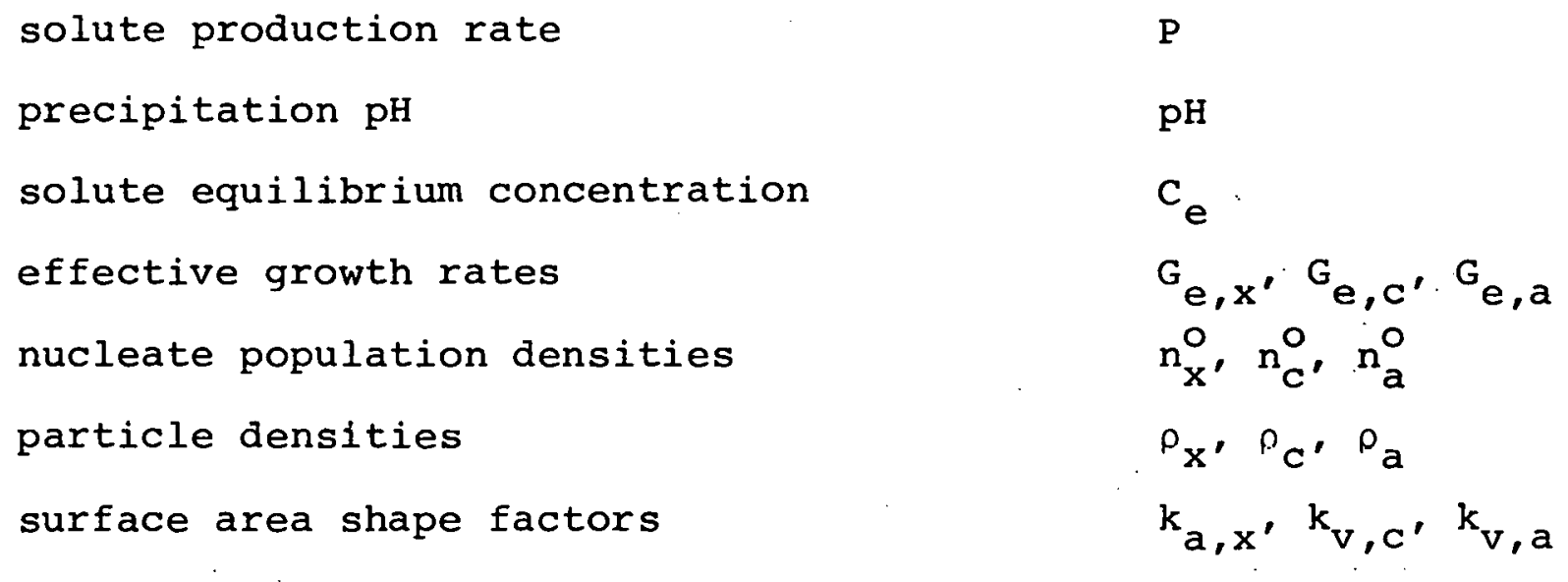

Parameters calculated from other terms

population density distributions

$n_{x}(L), n_{c}(L)$,

$n_{a}(I)$

average minimum sizes

$\overline{\mathrm{L}}_{m, c^{\prime}} \overline{\mathrm{L}}_{\mathrm{m}, \mathrm{a}}$

average nucleate population densities

$\overrightarrow{\mathrm{n}}_{\mathrm{c}^{\prime}}^{\circ}, \overrightarrow{\mathrm{n}}_{\mathrm{a}}^{\circ}$

average particle sizes

$\overline{\mathrm{L}}_{x^{\prime}}, \overline{\mathrm{L}}_{c^{\prime}}, \overline{\mathrm{L}}_{\mathrm{a}}$ 
Table 1. (Continued)

coagulation constants

$k_{x^{\prime}}^{\prime}, k_{x^{\prime}}^{\prime \prime} k_{C}$

growth rates

$G_{a}, G_{a}^{\prime}, G_{x}$

ãominant particle sizes

$L_{d, x}, L_{d, c}, L_{d, a}$

suspension densities

$M_{x}, M_{c}, M_{a}$

surface areas

$A_{a}, A_{c}, A_{a}$

total surface area

${ }^{\mathrm{T}}$

kinetic orders of nucleation

$i_{x^{\prime}} i_{c^{\prime}} i_{a^{\prime}}$

$j_{x^{\prime}} j_{c^{\prime}} j_{a}$

nucleation constants

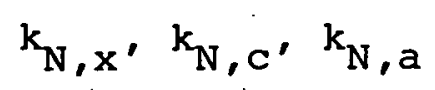


and shape factors $\left(k_{a, c}, k_{a, a}, k_{v, c}, k_{v, a}\right)$. The present work will allow the direct measurement of the effective crystallite growth rate and shape factors $\left(G_{e, x}, k_{a, x}\right.$ and $\left.k_{y, x}\right)$. However, since the electron microscope sample preparation method does not allow a reliable crystallite number density to be measured, a value for the crystallite nucleate population density $\left(n_{x}^{\circ}\right)$ cannot be directly obtained. Without this parameter the precipitation model cannot be completed. However, since the total suspension density of the reactor product is known, and Equations (35) and (36) express the cluster and agglomerate suspension densities as functions of known parameters, Equation (37) can be rearranged to solve for the crystallite suspension density.

$$
M_{x}=M_{T}-M_{C}-M_{a}
$$

Now Equation (34) can be used to solve for $n_{x}^{0}$. This completes the kinetic model for a continuous MSMPR precipitator as presented by Hoyt and applied to the continunus precipitation of ammonium polyuranate. 
EXPERIMENTAL PROCEDURE

All of the APU powder samples studied in this work were obtained from experimental runs made by Hoyt. For these runs filtered ammonium hydroxide and uranyl nitrate feeds were pumped continuously into an MSMPR precipitation vessel where they intimately mixed and reacted. The design of this precipitator followed that of a proven design from which crystal size distribution data conforms to the mixedsuspension, mixed product removal concept (16).

A diagram of the assembled precipitator is given in Figure 3. Feed solutions are introduced into the center of the draft tube where rapid dispersion occurs. The exact position of the product removal tube to permit isokinetic product removal and sampling was determined by observing the flow pattern of ion-exchange beads in the reactor and is such that the flow is uniformly vertical. An intermittent product removal system was used in order to avoid product removal velocities low enough that product classification might occur. The equipment flow sheet is shown in Figure 4 .

During Hoyt's experimental runs, steady state was reached between $1 \dot{2}$ and 15 residence times after which APU samples were taken from the product removal line. These samples were diluted with filtered, deionized water, filtered 


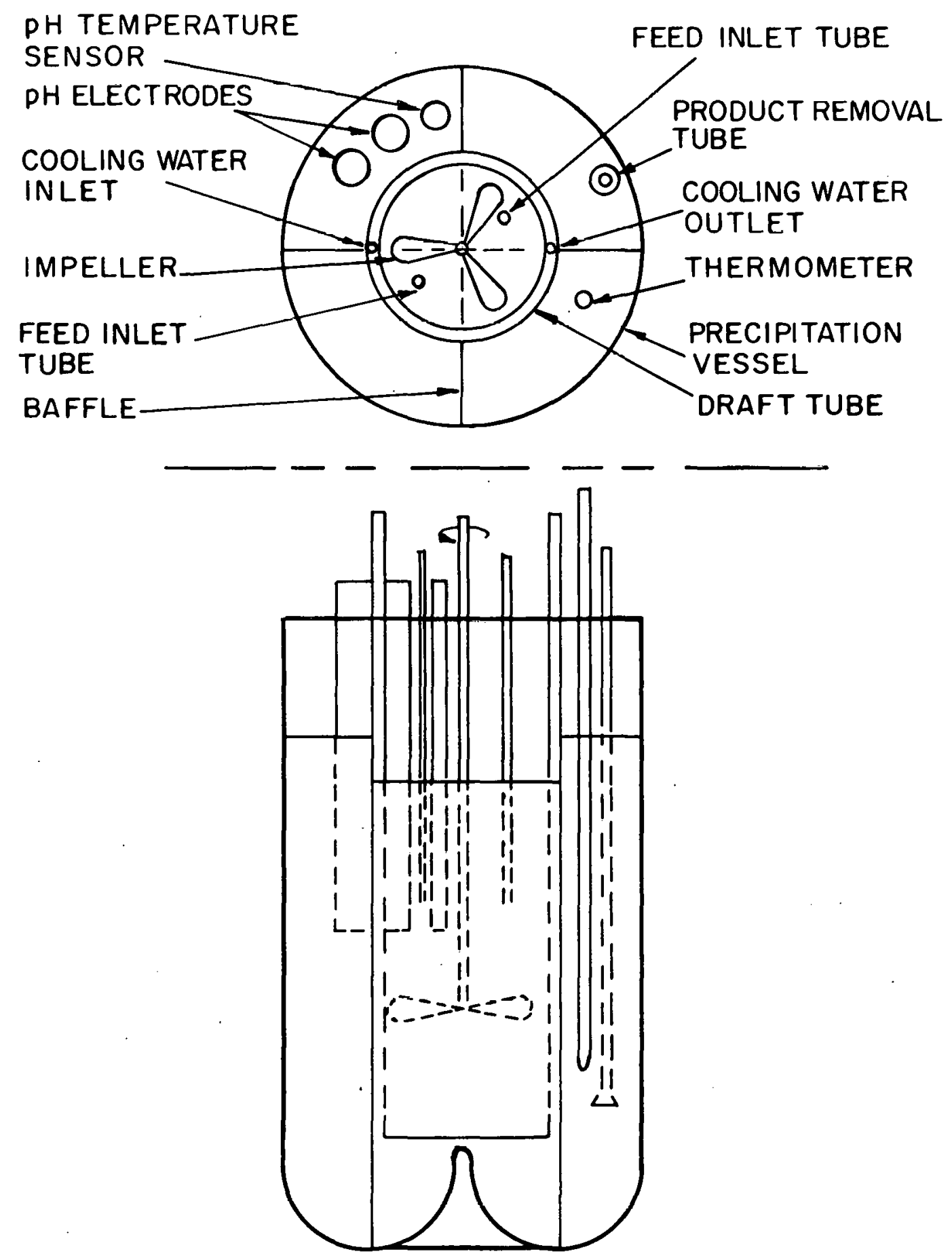

Figure 3. MSMPR precipitation reactor. 


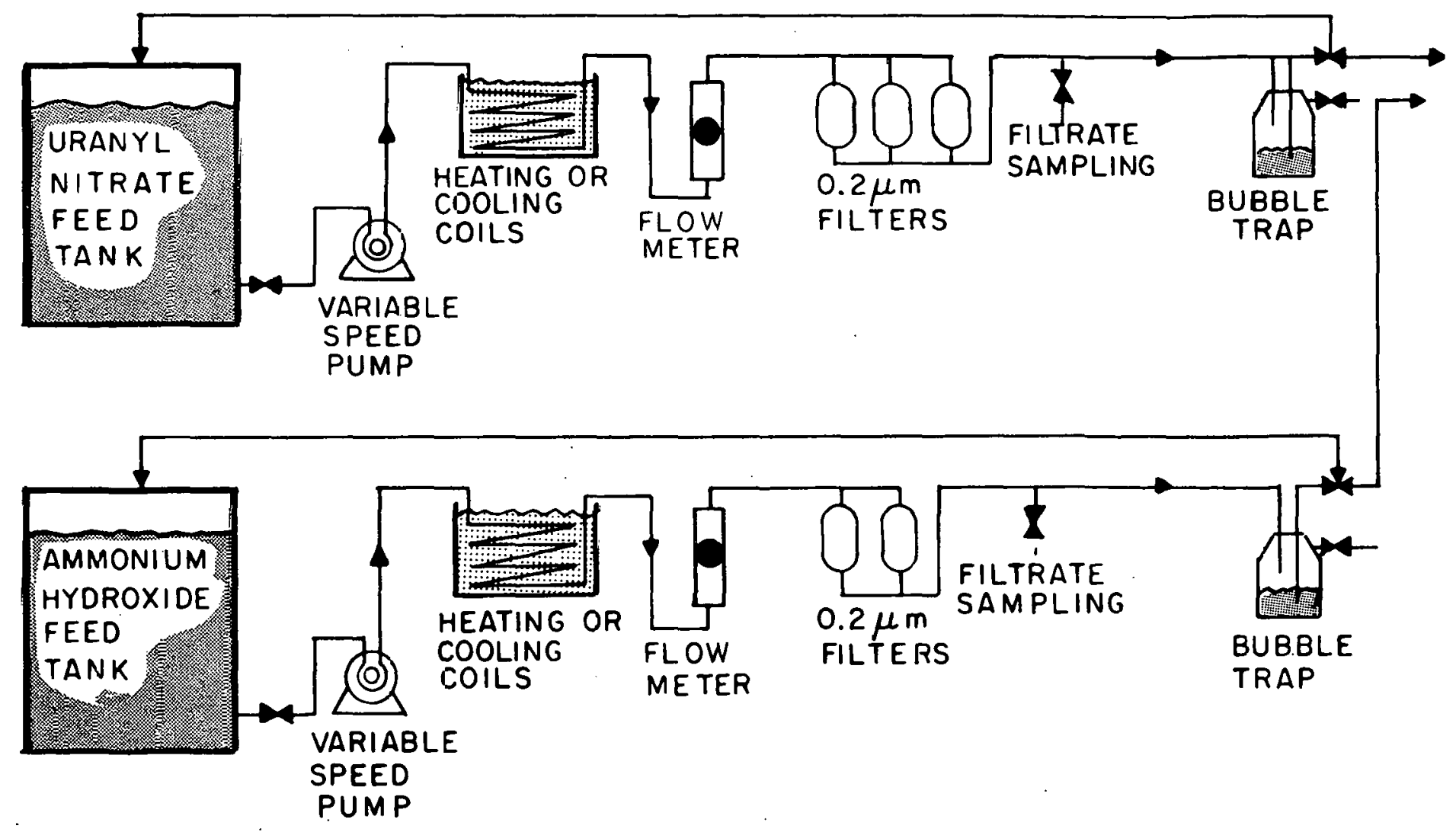

Figure 4. Equipment flow sheet. 
URANYL NITRATE FEED SOLUTION

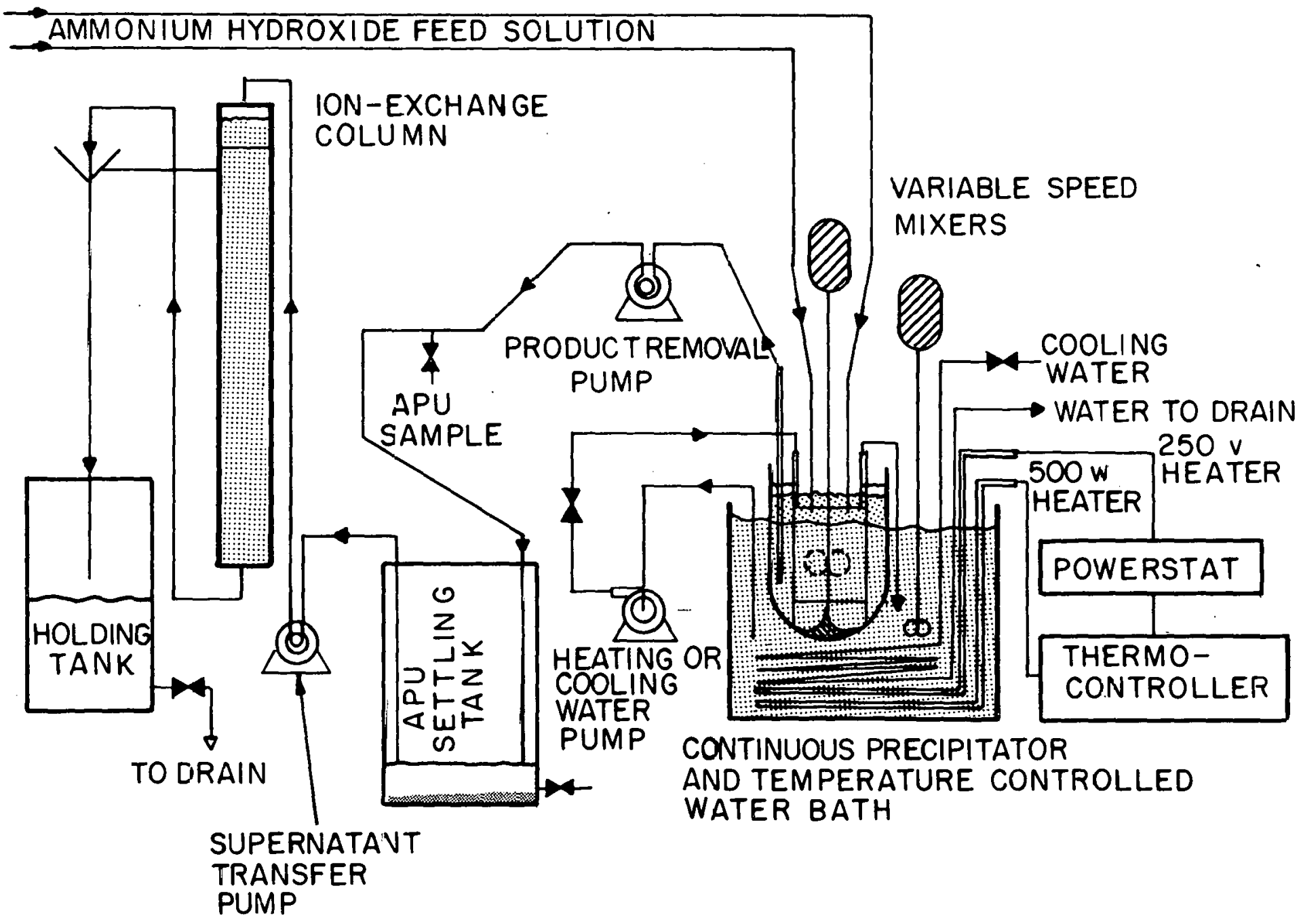

Figure 4. (Continued) 
through a $0.2 \mu \mathrm{m}$ millipore membrane, washed with water, and dried in a desiccator for several hours. Later, these samples were coated with approximately $200 \AA$ of gold and examined in the scanning electron microscope.

At the end of each reactor run a $30 \mathrm{ml}$ sample was removed from the precipitator, filtered, washed with alcohol, dried in air at $100^{\circ} \mathrm{C}$, and stored in a desiccator for later analysis with the transmission electron microscope. This filter cake was also analyzed with the SEM by sprinkling the powder cake onto a layer of wet silver paint and coating with gold after the paint had dried.

Preparation of the TEM samples from the APU filter cakes proved to be much more difficult. Each sample was prepared by dispersing 10 to 20 milligrams of the powder in approximately $2 \mathrm{ml}$ of filtered, deionized water by the use of extensive ultrasonics, centrifuging the solution long enough to remove the suspended APU clusters and agglomerates, and evaporating a drop of the resulting APU crystallite suspension onto a $2 \mathrm{~mm}, 200$ mesh copper grid coated with a thin carbon film. The APU crystallites, suspended on the carbon film across the mesh of the grid, were examined directly with the TEM for the purpose of obtaining a crystallite size distribution. It was found that the quantitative results that were obtained from this procedure were highly dependent upon the extent of treatment of the sample dispersion in both the ultrasonic bath and the centrifuge, both treatments having 
the tendency to classify crystallite sizes. As discussed later, a systematic study revealed the optimum experimental procedure to be used.

After obtaining the desired crystallite size measurements, the standard technique of metal shadow casting (27) was used in order to obtain the particle thickness and therefore a volumetric shape factor for the crystallites. For most of the samples the TEM micrographs revealed several crystallites standing on edge. This allowed crystallite thicknesses to be measured directly and the results compared to those obtained from the shadowing technique.

The APU powders were also examined by $x$-ray diffraction by way of the Debye-Scherrer powder diffraction method (11). 
DATA ANALYSIS

Scanning electron microscope studies were made of samples from all of the experimental runs of Hoyt. The microscope used was the JEOL JSM-U3 SEM shown in Figure 5. These studies were primarily an attempt at qualitatively describing the morphology of the powders as a function of the reacting conditions at which the powders were produced. A few of the powders were also studied quantitatively with the SEM by measuring the size distribution of both the clusters and agglomerates as they appeared on the electron micrographs. From these size distributions an effective growth rate was obtained for each type of particle and the results were compared to the growth rates obtained by Hoyt from Coulter counter measurements.

very little quantitative information about the crystallites could be obtained from the SEM, due to the small size of these particles. For this reason the transmission electron microscope was used to measure the size distribution and shape factors of the crystallites, thereby obtaining an effective crystallite growth rate for each APU sample. The Hitachi Type HU-125 TEM shown in Figure 6 was used for this study.

In order to obtain a particle size distribution from the electron micrographs the distinguishable particles on 


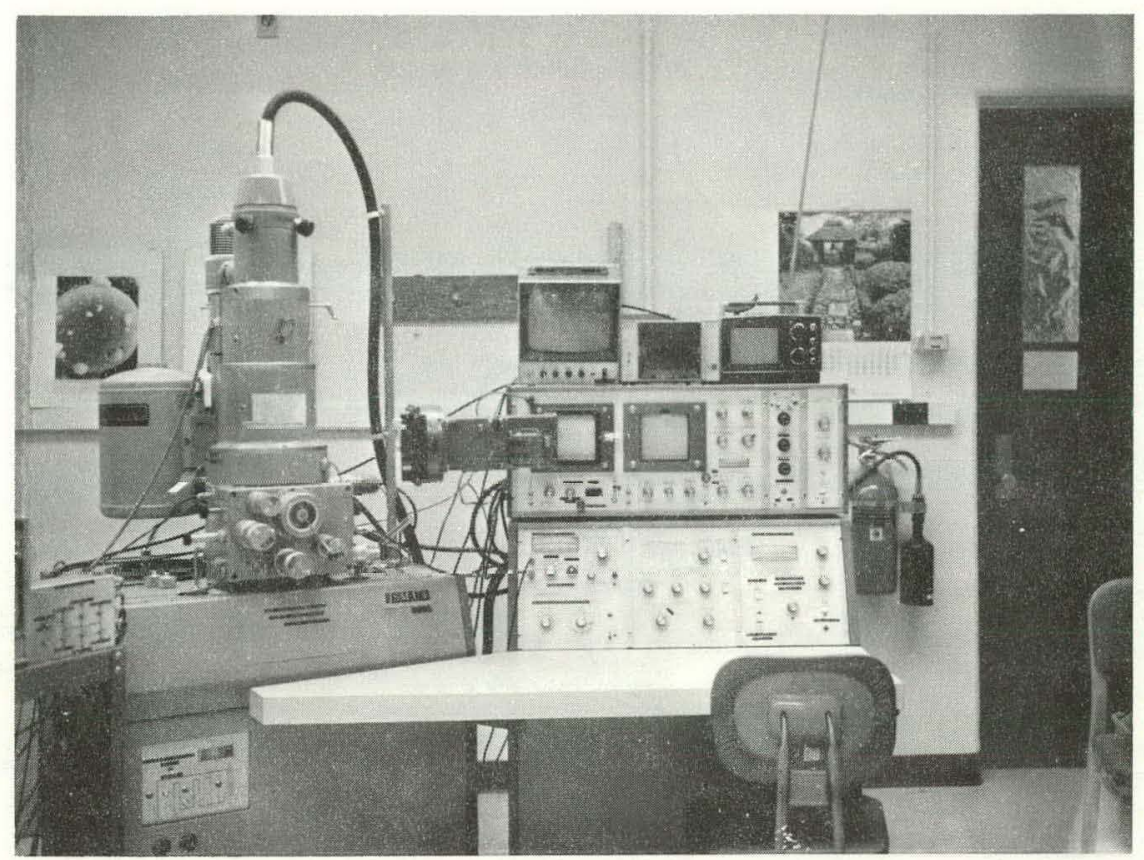

Figure 5. JEOL JSM-U3 scanning electron microscope.

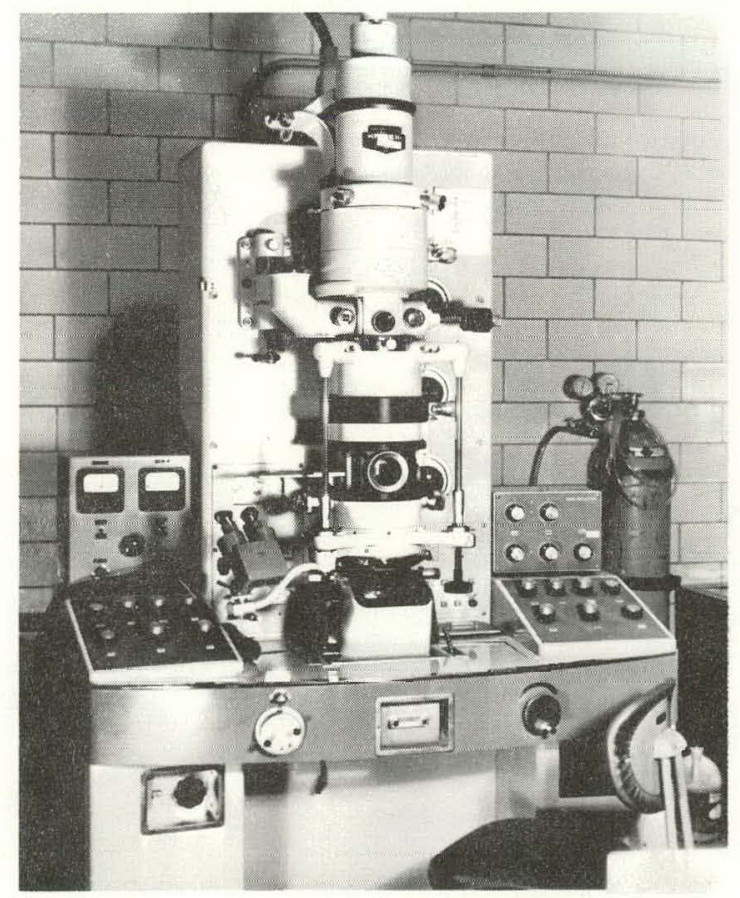

Figure 6. Hitachi Type HU-125 transmission electron microscope. 
the pictures were divided into size intervals by measuring the diameter of the circular equivalent area of the cross section of the particle by use of a circular metric template. The size intervals into which the observed particles had been divided were then converted to actual particle size intervals by use of the known magnification of the micrograph. Equation (9) indicates that a plot of the number density in each interval (number of particles in the interval/actual width of the interval in microns) vs. the mean size of the interval in microns on semilog paper should produce a straight line with a slope of $-1 / 2.303 \tau G_{e}$, assuming the effective growth rate and shape factors are size independent. Therefore, a least squares program was used to fit the best straight line to the data and an effective growth rate was obtained. It should be noted that because a value for the original suspension volume of the reactor product was not carried through to the number density calculations, the $y$-intercept of the fitted straight line of log of number density vs. particle size cannot be interpreted as $\log \mathrm{n}^{0}$ and another source must be found for the value of $n^{\circ}$.

The geometric shape factors for the crystallites were obtained by studying electron micrographs of TEM samples that had been shadowed with approximately $50 \AA$ of germanium metal at a $15^{\circ}$ angle. 
X-ray powder patterns were obtained for each APU powder sample by the use of a 6 inch Debye-Scherrer camera and a Cuka radiation source. The resulting diffraction lines were measured with a linear scale, converted to values of $2 \theta$ and $\sin ^{2} \theta$, and compared to the values reported by Debets (12) for phases present in the $\mathrm{NH}_{3}-\mathrm{UO}_{3}-\mathrm{H}_{2} \mathrm{O}$ system. 


\section{RESULTS AND DISCUSSION}

Hoyt (18) operated the continuous APU precipitation process for 20 experimental runs, the conditions of which are listed in Table 2. The precipitations were done at room temperature $\left(25\right.$ to $\left.27^{\circ} \mathrm{C}\right)$ to avoid difficulties in maintaining the APU samples at the operating temperature during sample analysis with the Coulter counter. A residence time of 13 minutes was chosen so that steady state conditions would be reached within a reasonable length of time (2.5 to $3.5 \mathrm{hrs})$. The first 14 runs involved changes in the $\mathrm{NH}_{3}: \mathrm{U}$ reacting feed mole ratios (RFMR) for a given constant uranium concentration. This is equivalent to changing the $\mathrm{pH}$ of the reacting mixture at constant uranium concentration. The correlation between $\mathrm{pH}$ and RFMR is given in Figure 1. Runs number 18 to 20 involved changes in the uranium conconcentration at constant RFMR.

Several of the samples from Hoyt's work are not analyzed in parts of this work for varying reasons. Runs number 3, 15 and 16 involved equipment failures of one sort or another before steady state was obtained. Run number 17 varied in both uranium concentration and RFMR from all other experimental runs. Samples from runs number 1 and number 5 were not available for analysis. 
Table 2. Experimental conditions for APU precipitations

uranyl nitrate flow rate $=140 \mathrm{ml} / \mathrm{min}$

ammonium hydroxide flow rate $=17.8 \mathrm{ml} / \mathrm{min}$

suspension hold up volume $=2050 \mathrm{ml}$

residence time $=13.0 \mathrm{~min}$

stirring rate $=1200 \mathrm{rpm}$

\begin{tabular}{|c|c|c|c|c|c|}
\hline $\begin{array}{l}\text { Ruin } \\
\text { Number }\end{array}$ & $\begin{array}{c}\mathrm{U} \\
(\mathrm{moles} / \ell)\end{array}$ & $\begin{array}{c}\mathrm{NH}_{3} \\
(\mathrm{moles} / \ell)\end{array}$ & RFMR & $(\stackrel{\circ}{\circ})$ & $\left(\right.$ at $\left.25^{\circ} \mathrm{C}\right)$ \\
\hline 1 & 0.0249 & 0.4602 & 2.70 & 25.0 & 8.60 \\
\hline 2 & 0.0249 & 0.4367 & 2.58 & 25.2 & 8.50 \\
\hline 3 & 0.0249 & 0.2682 & 2.23 & 25.0 & 7.51 \\
\hline 4 & 0.0249 & 0.3995 & 2.39 & 25.5 & 8.15 \\
\hline 5 & 0.0249 & 0.3956 & 2.37 & 25.5 & 8.12 \\
\hline 6 & 0.0249 & 0.3896 & 2.34 & 25.5 & 8.00 \\
\hline 7 & 0.0249 & 0.3593 & 2.20 & 25.0 & 7.10 \\
\hline 8 & 0.0249 & 0.3593 & 2.19 & 25.5 & 7.13 \\
\hline 9 & 0.0249 & 0.3691 & 2.23 & 26.0 & 7.50 \\
\hline 10 & 0.0249 & 0.3769 & 2.28 & 26.0 & 7.78 \\
\hline 11 & 0.0249 & 0.3437 & 2.08 & 25.5 & 5.84 \\
\hline 12 & 0.0249 & 0.3515 & 2.15 & 25.5 & 6.49 \\
\hline 13 & 0.0249 & 0.3554 & 2.17 & 26.0 & 6.80 \\
\hline 14 & 0.0249 & 0.6132 & 3.47 & 26.0 & 9.00 \\
\hline 15 & 0.2000 & 2.886 & 2.18 & 27.0 & 4.60 \\
\hline 16 & 0.0025 & 0.0361 & 2.19 & 25.0 & 5.47 \\
\hline 17 & 0.0025 & 0.0616 & 3.73 & 25.5 & 8.88 \\
\hline
\end{tabular}


Table 2. (Continued)

\begin{tabular}{llllll}
\hline 18 & 0.2000 & 4.926 & 3.48 & 27.5 & 9.0 \\
19 & 0.0100 & 0.246 & 3.48 & 25.5 & 8.27 \\
20 & 0.1000 & 2.463 & 3.48 & 26.0 & 9.10 \\
\hline
\end{tabular}


Characterization of APU Particle structures

For each experimental run the coulter counter analysis of the precipitate produced a population density distribution (PDD). Figure 7 shows the extremes of the PDD obtained through changes in the RFMR for a 0.0249 molar uranium concentration. The shape of the PDD from run number 14 $($ RFMR $=3.47, \mathrm{pH}=9.00)$ is typical of those obtained when precipitating at high $\mathrm{pH}$ values $(\mathrm{RFMR} \geq 2.34, \mathrm{pH} \geq 8.00)$; and the shape of the PDD from run number 7 is typical of those obtained when precipitating at low $\mathrm{pH}$ values (RFMR < $2.34, \mathrm{pH}<8.00)$.

The measured PDD for low values of RFMR and $\mathrm{pH}$ result from the sum of two size-independent particle growth processes, as indicated. in Figure 8 . It is postulated that these two processes are actually primary and secondary coagulation, rather than some type of size-dependent process. The kinetic results from the coulter counter analysis of the continuous precipitation experiments are listed in Table 3. These results were obtained by fitting a straight line to each of the two size-independent growth processes observed from the PDD for each experimental run, as is shown in Figure 8. Ideally, the PDD should show three sizeindependent growth processes. However, since the crystallite sizes are below the range of the coulter counter the 


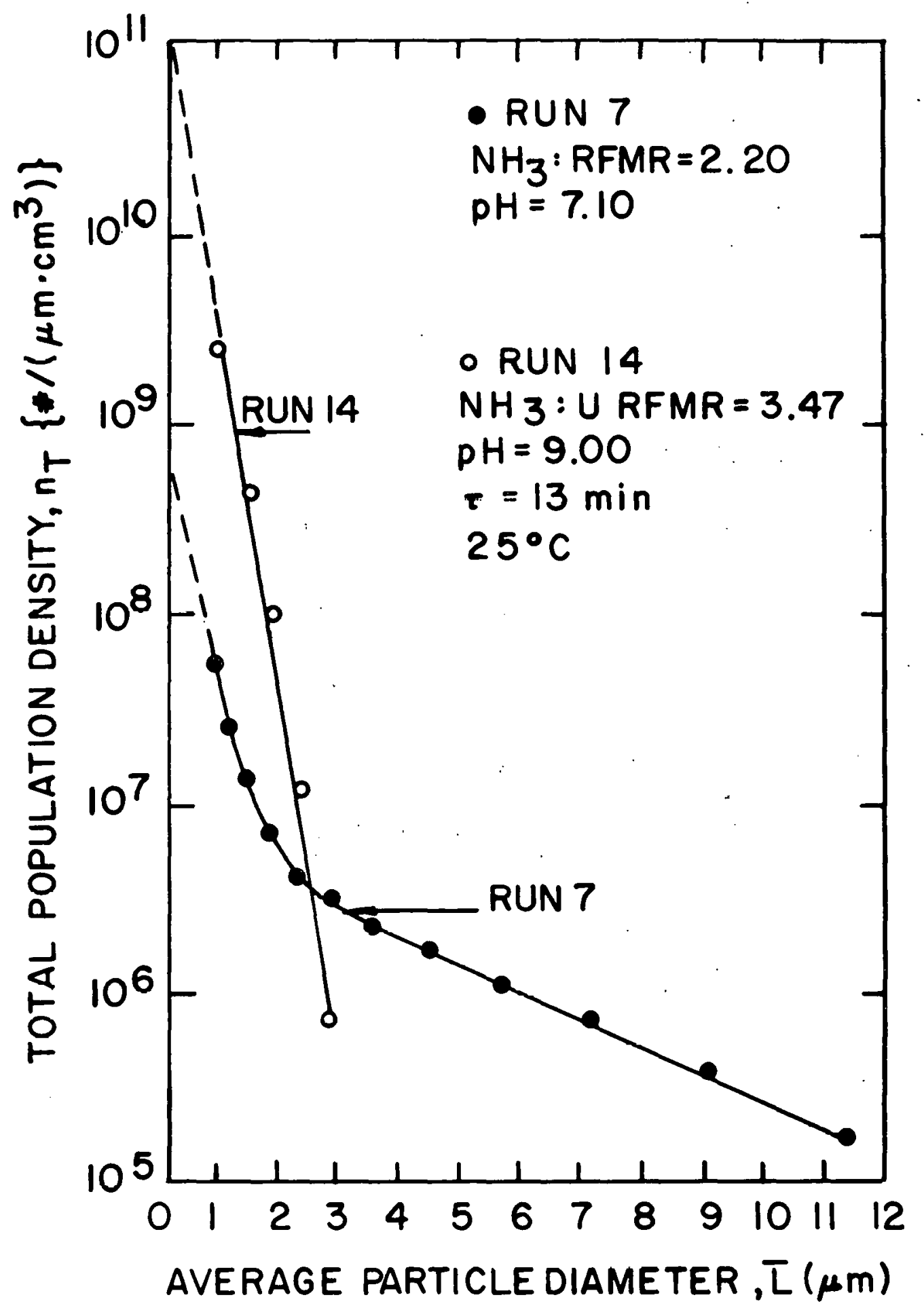

Figure 7. Population density distribution extremes for precipitation at a constant uranium concentration of 0.0245 molar. 


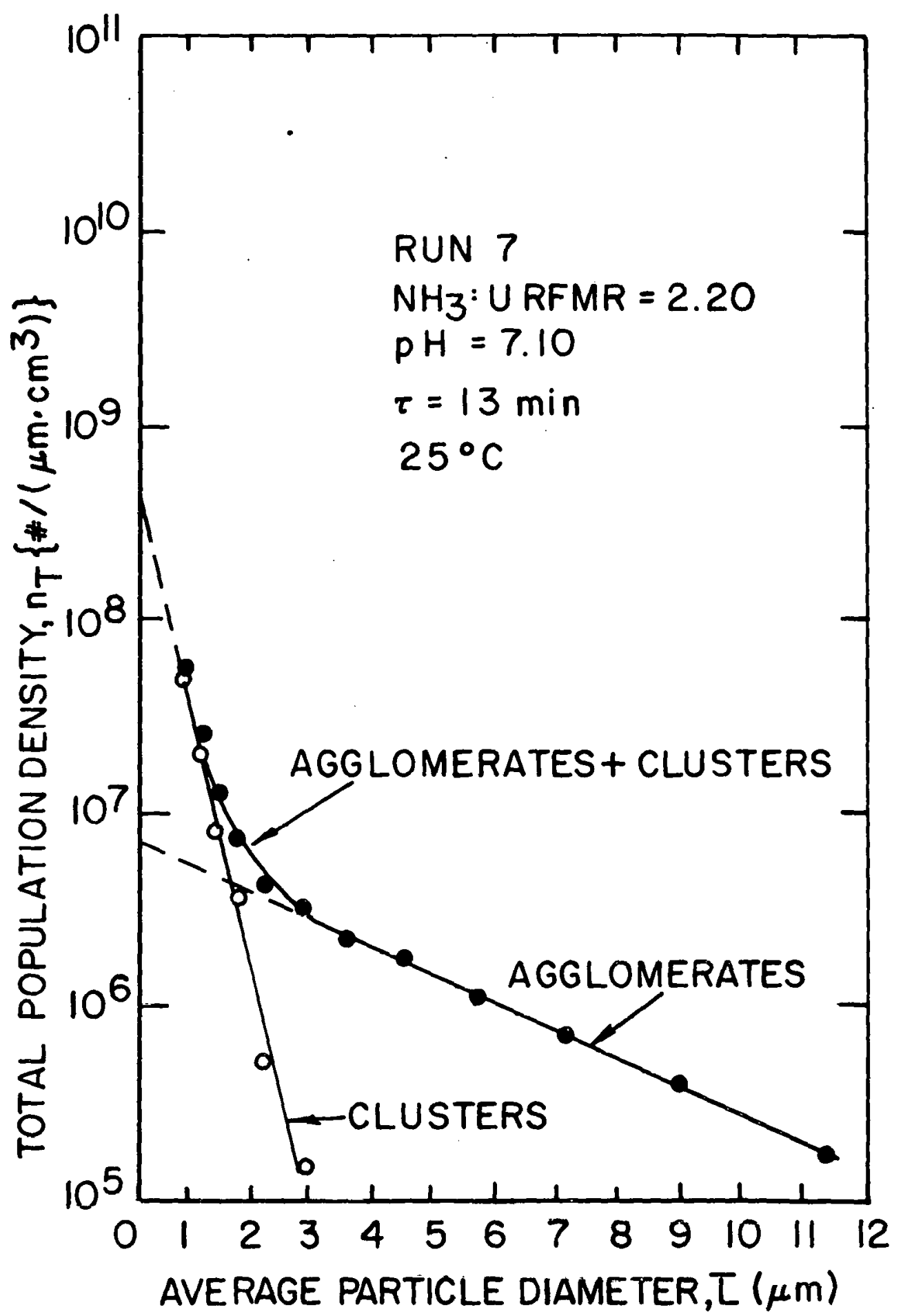

Figure 8. Resolution of the population density distribution for run 7 , shown in Figure 7 , into two sizeindependent growth processes. 
Table 3. Kinetic results from experimental runs

\begin{tabular}{|c|c|c|c|c|}
\hline $\begin{array}{l}\text { Run } \\
\text { Number }\end{array}$ & $\begin{array}{c}\text { Type of } \\
\text { Particles }\end{array}$ & $\begin{array}{c}\mathrm{n}^{0} \times 10^{-8} \\
\# /\left(\mu \mathrm{m} \cdot \mathrm{cm}^{3}\right)\end{array}$ & $\begin{array}{l}\mathrm{G}_{\mathrm{e}} \times 10^{+4} \\
(\mu \mathrm{m} / \mathrm{sec})\end{array}$ & $\begin{array}{c}\mathrm{B}^{\circ} \times 10^{-5} \\
\# /\left(\mathrm{cm}^{3} \cdot \mathrm{sec}\right)\end{array}$ \\
\hline 2 & $\mathrm{a}$ & 322 & 4.46 & 144 \\
\hline 4 & $a$ & 14.7 & 6.87 & 28.7 \\
\hline 6 & $a$ & 13.7 & 9.27 & 12.7 \\
\hline 7 & $\begin{array}{l}c \\
a\end{array}$ & $\begin{array}{l}6.64 \\
0.0773\end{array}$ & $\begin{array}{l}4.23 \\
38.7\end{array}$ & $\begin{array}{l}2.81 \\
0.299\end{array}$ \\
\hline 8 & $\begin{array}{l}\text { c } \\
\text { a }\end{array}$ & $\begin{array}{l}6.95 \\
0.0740\end{array}$ & $\begin{array}{r}4.51 \\
39.4\end{array}$ & $\begin{array}{l}3.13 \\
0.292\end{array}$ \\
\hline 9 & $\begin{array}{l}c \\
a\end{array}$ & $\begin{array}{l}9.84 \\
0.166\end{array}$ & $\begin{array}{r}4.77 \\
32.7\end{array}$ & $\begin{array}{l}4.69 \\
0.541\end{array}$ \\
\hline 10 & $\begin{array}{l}\mathrm{c} \\
\mathrm{a}\end{array}$ & $\begin{array}{c}31.4 \\
1.79\end{array}$ & $\begin{array}{l}3.47 \\
17.1\end{array}$ & $\begin{array}{l}10.9 \\
3.05\end{array}$ \\
\hline 11 & $\begin{array}{l}\text { c } \\
\text { a }\end{array}$ & $\begin{array}{l}7.02 \\
0.773\end{array}$ & $\begin{array}{r}3.71 \\
25.8\end{array}$ & $\begin{array}{l}2.60 \\
2.00\end{array}$ \\
\hline 12 & $\begin{array}{l}\mathrm{c} \\
\mathrm{a}\end{array}$ & $\begin{array}{l}3.60 \\
0.167\end{array}$ & $\begin{array}{l}4.34 \\
37.2\end{array}$ & $\begin{array}{l}1.56 \\
0.619\end{array}$ \\
\hline 13 & $\begin{array}{l}\mathrm{c} \\
\mathrm{a}\end{array}$ & $\begin{array}{l}6.02 \\
0.0912\end{array}$ & $\begin{array}{r}3.96 \\
38.9\end{array}$ & $\begin{array}{l}2.38 \\
0.355\end{array}$ \\
\hline 14 & c & 1,440 & 3.06 & 440 \\
\hline 18 & $\begin{array}{l}\mathrm{c} \\
\mathrm{a}\end{array}$ & $\begin{array}{r}9,470 \\
\quad 0.774\end{array}$ & $\begin{array}{l}2.68 \\
7.68\end{array}$ & $\begin{array}{l}2,540 \\
0.594\end{array}$ \\
\hline 18 & c & 11.4 & 9.36 & 0.107 \\
\hline 20 & & 5,010 & 3.29 & 1,650 \\
\hline
\end{tabular}


crystallite growth process is not observed. From the slope of each of the lines observed an effective particle growth rate, $G_{e}$, is obtained for the respective growth processes. Each straight line can also be extrapolated to zero size in order to obtain the nucleate population density, $\mathrm{n}^{\circ}$, for the respective growth process. At high $\mathrm{pH}$ values the two observed particle growth processes become indistinguishable and the PDD appears as one straight line, as is shown for run number 14 in Figure 7 . In such cases the kinetic results are reported as a single growth process. The postulation that the two particle growth processes are actually primary and secondary coagulation, rather than some type of size-dependent process, is supported not only by the shape of the PDD, but also through observations of the precipitate with the scanning electron microscope (SEM). Figures 9 through 17 are SEM micrographs of several of the APU samples at the same uranium concentration (0.0249 molar). The photographs are arranged here in the order of increasing RFMR (or increasing $\mathrm{pH}$ ) for runs.

Several general characteristics of the powders can be observed in these photographs. At a RFMR $\leq 2.15(\mathrm{pH} \leq 6.5)$ the precipitate is made up of large dense agglomerates, ranging from approximately 1 to $10 \mathrm{\mu m}$ in diameter, which are composed of smaller clusters, with typical sizes of 0.1 to $1.0 \mu \mathrm{m}$. These features can be seen in Figures 9 and 


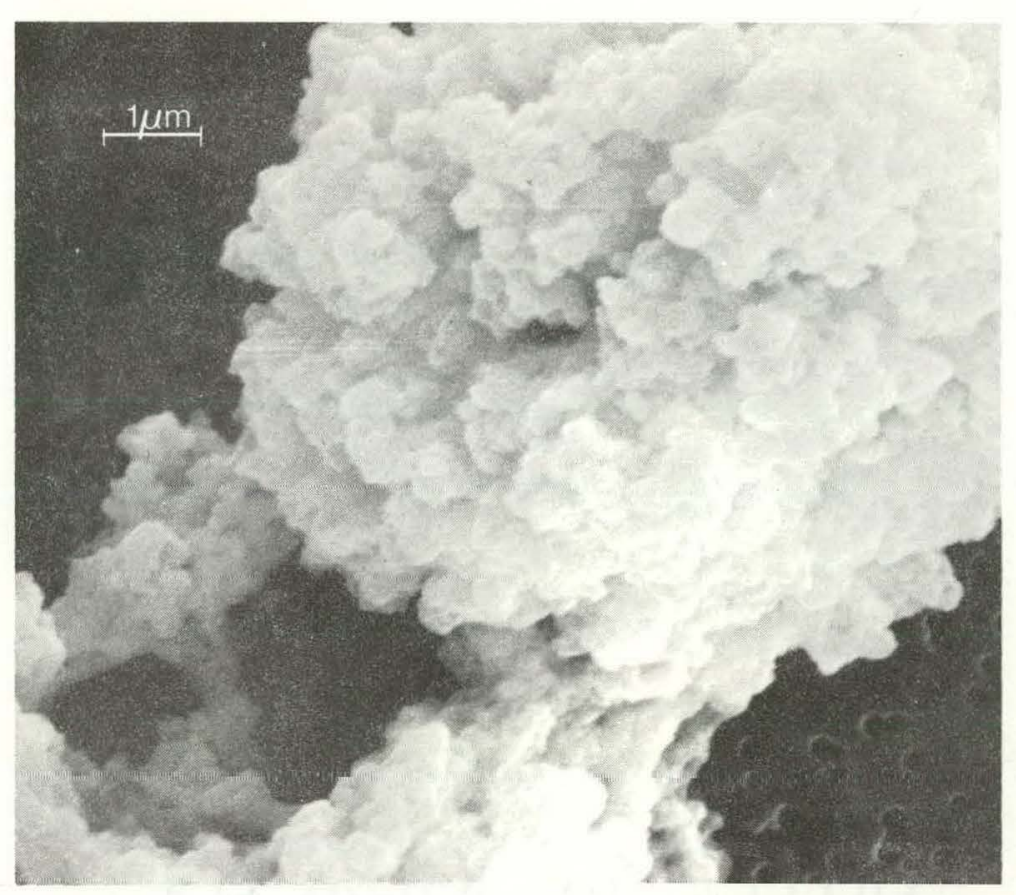

Figure 9. SEM micrograph $(10,000 \mathrm{X})$ of APU from run number 11 $($ RFMR $=2.08, \mathrm{pH}=5.84)$.

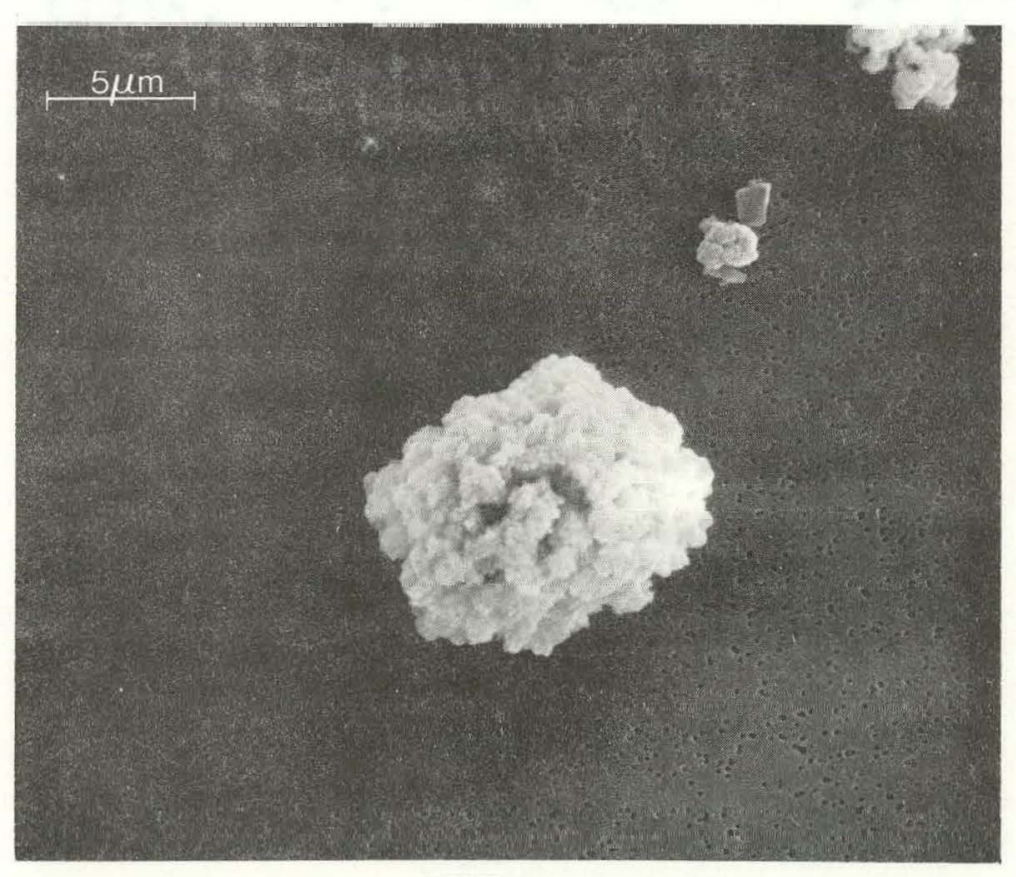

Figure 10. SEM micrograph $(3,000 \mathrm{X})$ of APU from run number 12 $($ RFMR $=2.15, \mathrm{pH}=6.49)$. 
10 which are samples from run number 11 (RFMR $=2.08, \mathrm{pH}=$ $5.84)$ and run number 12 (RFMR $=2.15, \mathrm{pH}=6.49)$ respectively. The samples were prepared on millipore membranes. There appears to be considerable pore volume within the agglomerates but minimal void space within the individual clusters. Figure 11 is a picture of a filter cake sample from run number 11. The individual crystallites, which are not easily detectable in Figures 9 and 10, are observed in Figure 11 to be very thin platelets with characteristic lengths of less than $0.5 \mu \mathrm{m}$.

At a RFMR between 2.15 and $2.30(\mathrm{pH}=6.50$ to 7.85$)$ the agglomerates and clusters appear to be much more loosely packed, as seen in Figure 12 of run number 13 (RFMR $=2.17$, $\mathrm{pH}=6.80)$ and Figure 13 of run number $10(\mathrm{RFMR}=2.28$, $\mathrm{pH}=7.78)$.

At a RFMR of 2.30 to $2.40(\mathrm{pH}=7.85$ to 8.15$)$ the agglomerates become so loosely packed that they collapse on the membrane surface as shown in Figure 14 of run number $6($ RFMR $=2.34, \mathrm{pH}=8.00)$. The structure of the clusters are also much less distinct than at low $\mathrm{pH}$ values. Figure 15 is a higher magnification of the same sample and shows the individual crystallites to be smaller than those of run number 11 observed in Figure 11.

At a RFMR greater than $2.40(\mathrm{pH}>8.15)$ the agglomerate structure is no longer detectable. Figure 16 which is a 


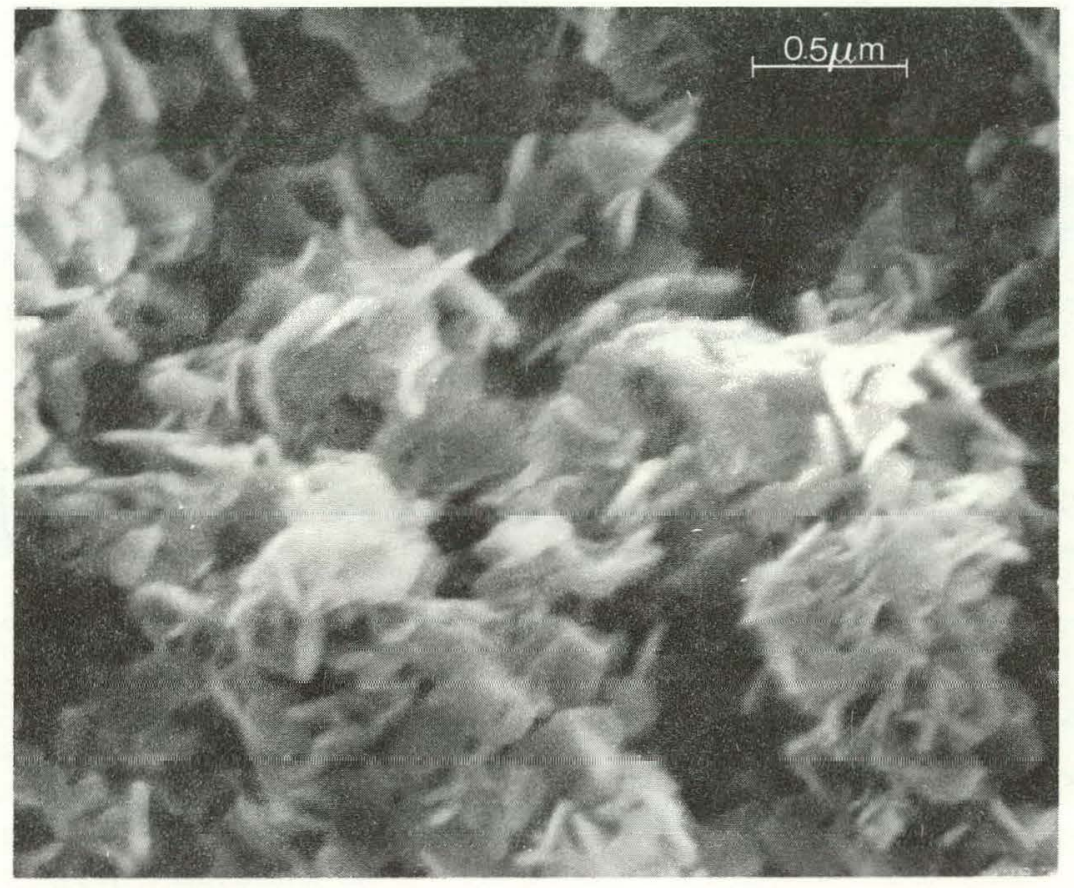

Figure 11. SEM micrograph $(30,000 \mathrm{X})$ of APU filter cake from run number $11(\mathrm{RFMR}=2.08, \mathrm{pH}=5.84)$. 


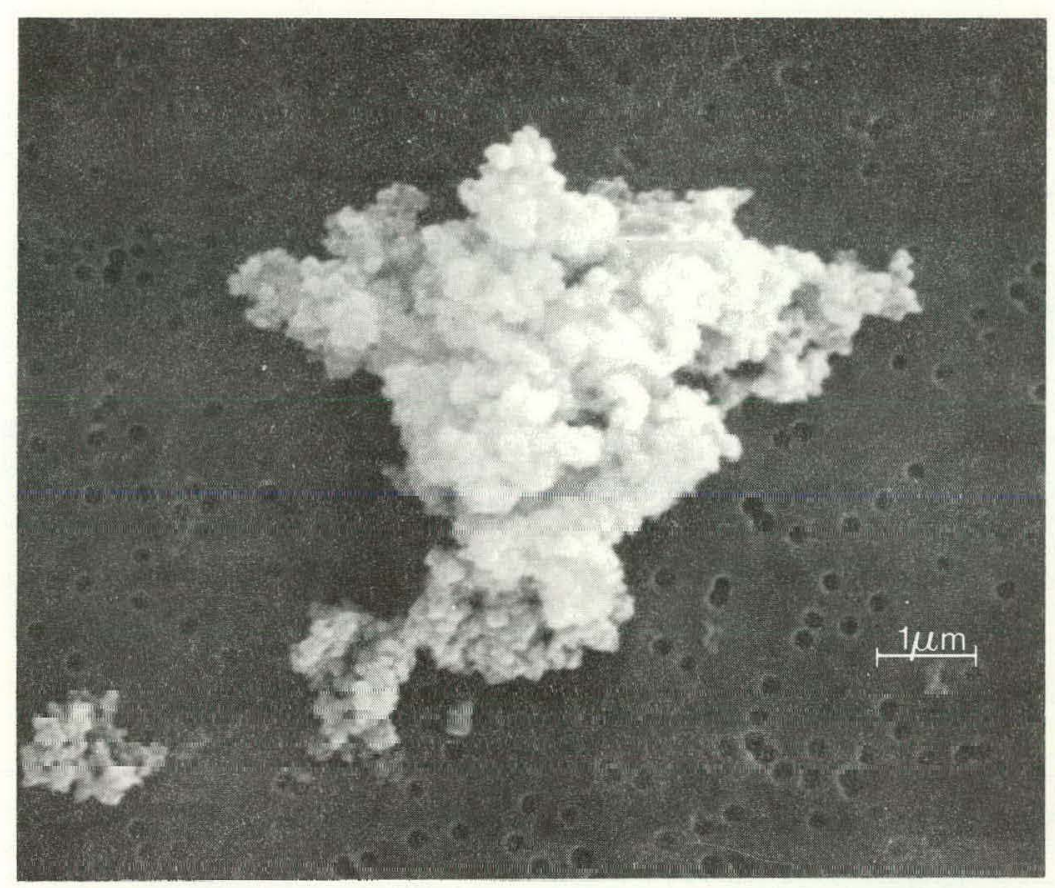

Figure 12. SEM micrograph $(10,000 \mathrm{X})$ of APU from run number $13(\mathrm{R} F \mathrm{MR}=2.17, \mathrm{pH}=6.80)$.

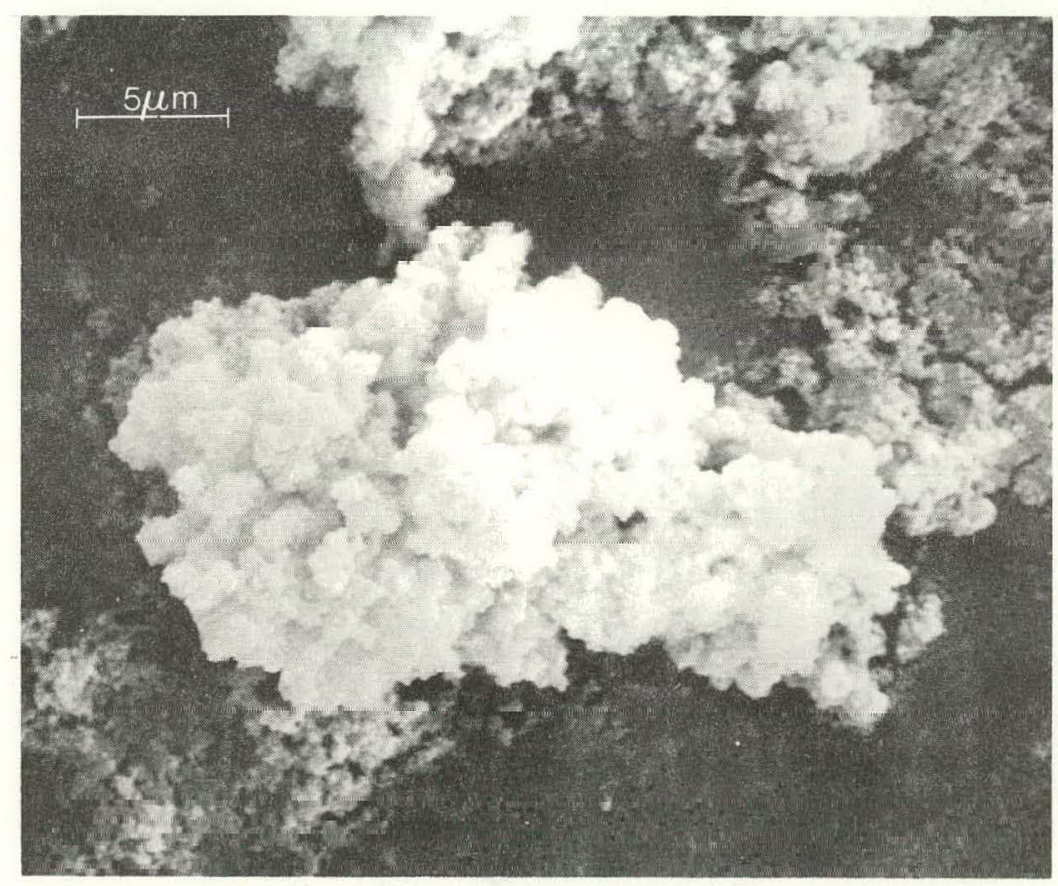

Figure 13. SEM micrograph $(3,000 \mathrm{X})$ of APU from run number $10($ RFMR $=2.28, \mathrm{pH}=7.78)$. 


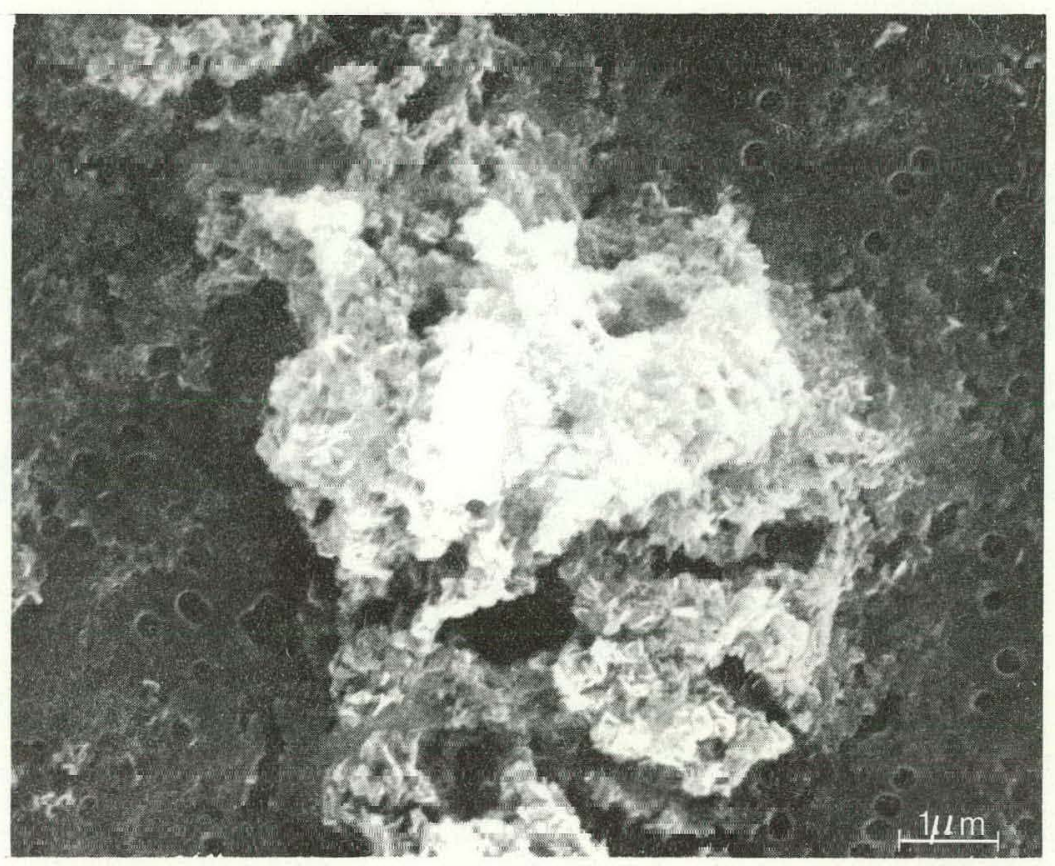

Figure 14. SEM micrograph $(10,000 \mathrm{X})$ of APU from run number 6 $(\mathrm{RFMR}=2.34, \mathrm{pH}=8.00)$.

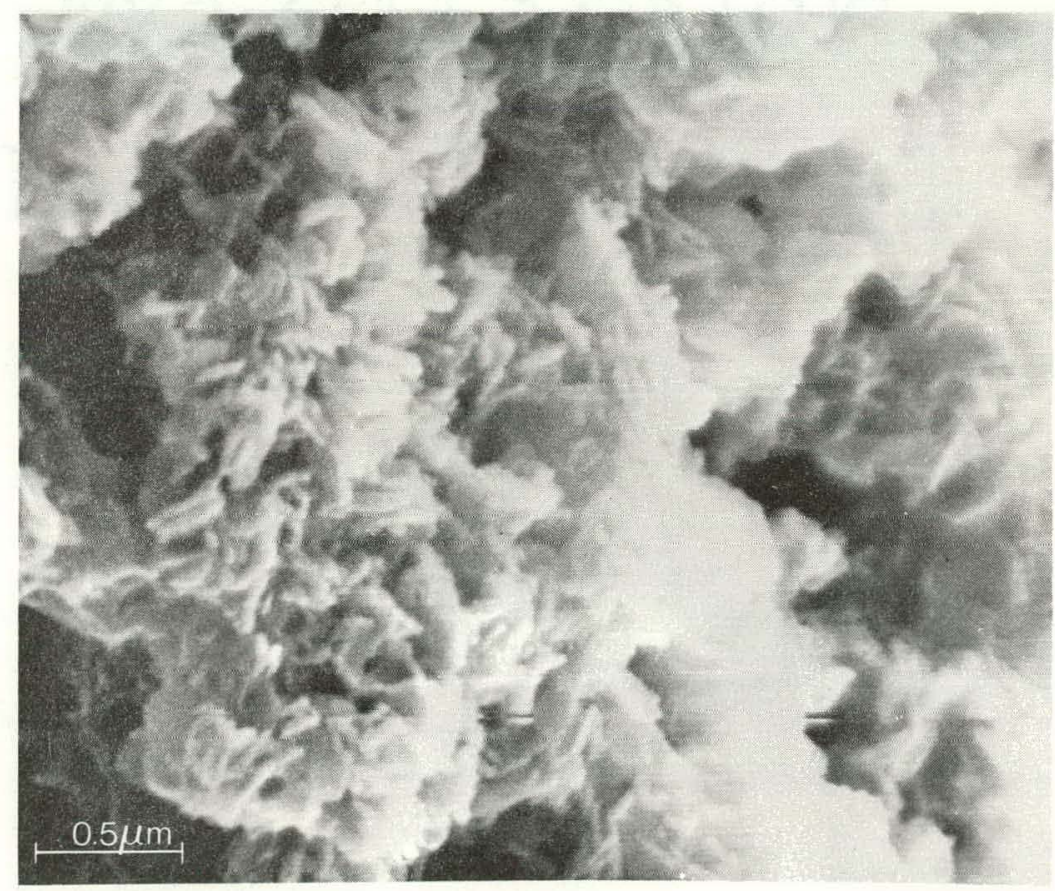

Figure 15. SEM micrograph $(30,000 \mathrm{X})$ of APU from run number 6 $($ RFMR $=2.34, \mathrm{pH}=8.00)$. 
filter cake sample of run number 2 (RFMR $=2.58, \mathrm{pH}=8.50)$ shows a cake of very loosely packed crystallites. Figure 17 is a much higher magnification of a filter cake from run number $14($ RFMR $=3.47, \mathrm{pH}=9.00)$. The individual crystallites appear to be of a larger size than those observed in Figures 11 or 14. This relative comparison of crystallite sizes is totally consistent with the crystallite growth rates to be reported later.

The Coulter counter results agree well with the observations made above. At low values of RFMR and $\mathrm{pH}$ two distinct size-independent growth processes are observed, in agreement with the two types of particles observed in the SEM micrographs. From the kinetic results listed in Table 3 an approximate value for the mean particle sizes can be obtained from an approximation of Equations (23) and (32),

$$
\bar{L}=\tau G_{e}
$$

where the average minimum particle sizes, $\overline{\mathrm{L}}_{\mathrm{m}}$, are approximated to be zero. From the kinetic results listed for run number 11 values for the mean particle sizes are calculated to be $0.29 \mu \mathrm{m}$ and $2.0 \mu \mathrm{m}$ for the clusters and agglomerates respectively. This is in close agreement with the observations made from Figure 9. At values of RFMR greater than 2.3 and $\mathrm{pH}$ above 7.8 it is difficult to distinguish between 


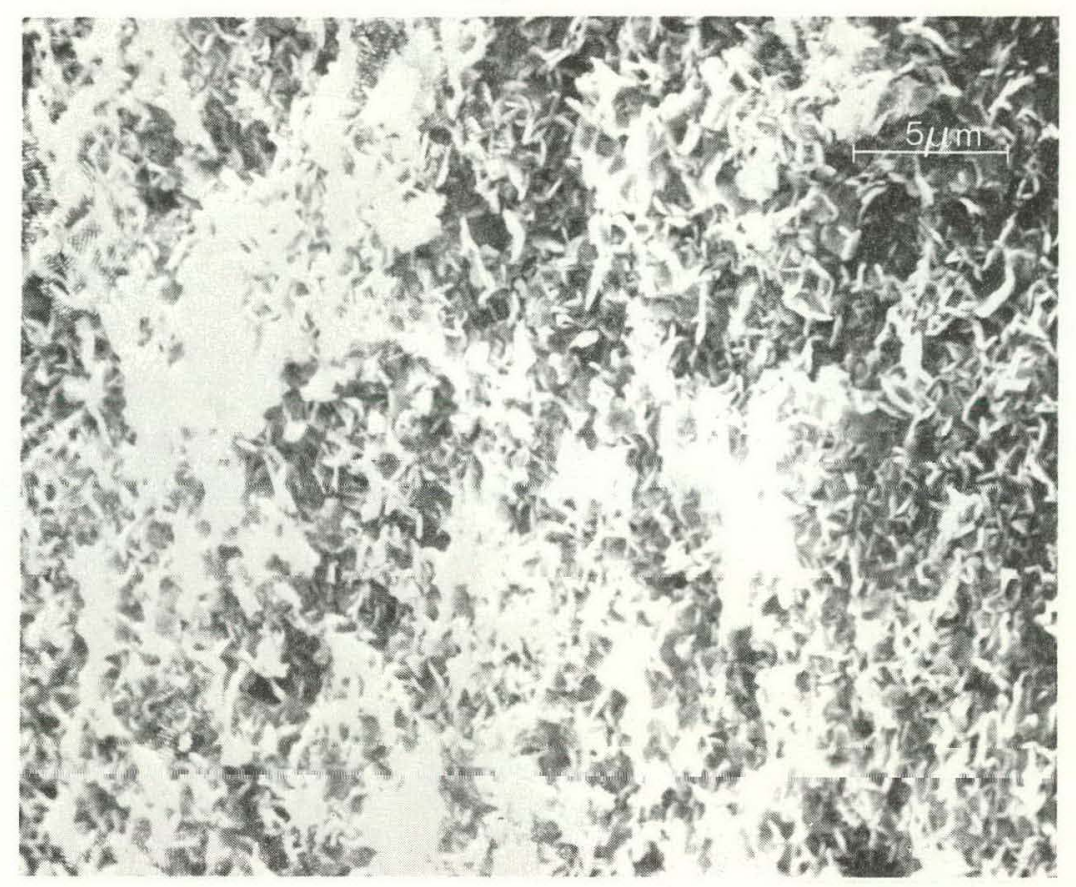

Figure 16. SEM micrograph $(3,000 \mathrm{X})$ of APU filter cake from run number 2 ( $R F M R=2.58, \mathrm{pH}=8.50)$.

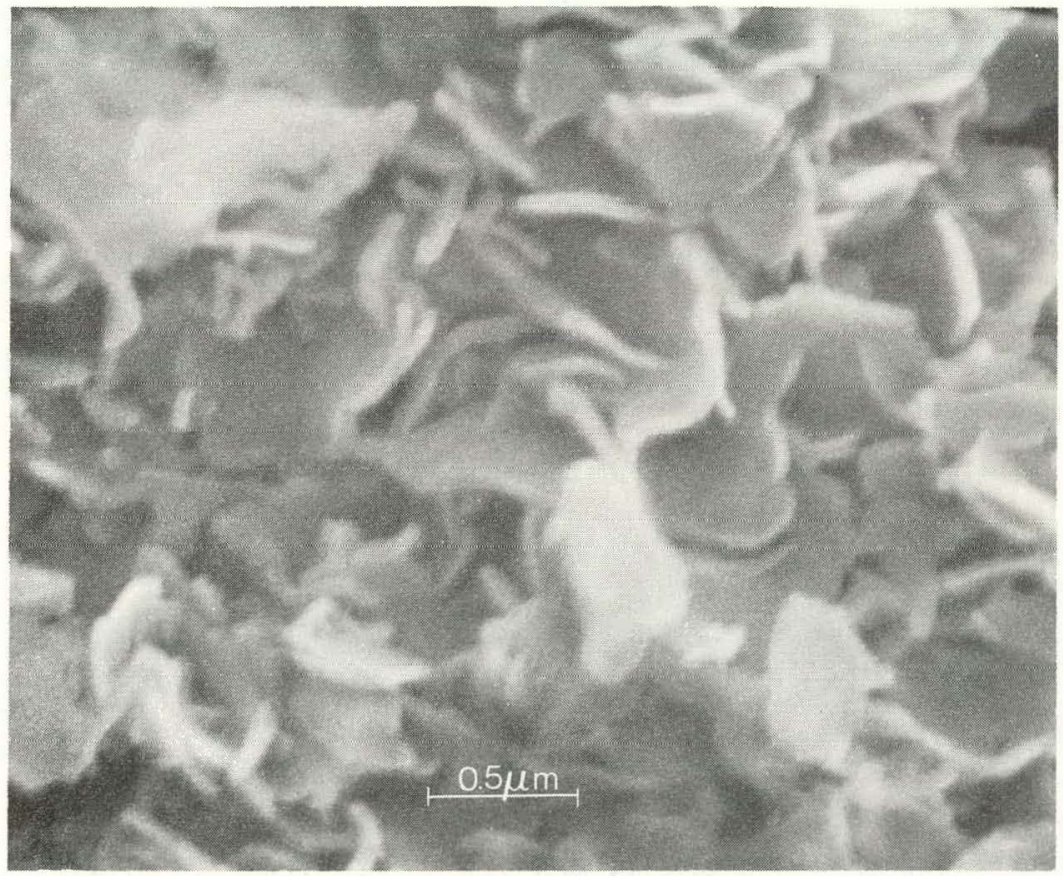

Figure 17. SEM micrograph $(30,000 \mathrm{x})$ of APU filter cake from run number 14 (RFMR $=3.47, \mathrm{pH}=9.00)$. 
the primary and secondary coagulation processes by either the coulter counter results or the SEM micrographs.

The effect of uranium concentration upon the particle characteristics can be observed from SEM micrographs of samples produced at varying uranium concentrations but at the same RFMR. Figures 18 and 19 are micrographs of samples number $15(\mathrm{U}=0.200$ molar $, \mathrm{pH}=4.60, \mathrm{RFMR}=2.18)$ and number $16(\mathrm{U}=0.0025$ molar $, \mathrm{pH}=5.47, \operatorname{RFMR}=2.19)$, respectively. In both cases the particle characteristics are typical of those observed earlier for the runs made at low values of RFMR and $\mathrm{pH}$. However, a distinct variation exists in the particle sizes of the two samples with both the clusters and agglomerates in the sample from run number 15 appearing to be 2 to 3 times larger than the respective particles in the sample from run number 16. This again is in close agreement with the kinetic results reported in Table 3 from which the ratio of particle growth rates from run number 15 to those from run number 16 are found to be 2.5 and 1.9 for the agglomerates and clusters, respectively. The results indicate that the reacting uranium concentration can give a noticeable effect on the particle sizes, but that RFMR and $\mathrm{pH}$ are the dominant factors in determining the particle morphology. 


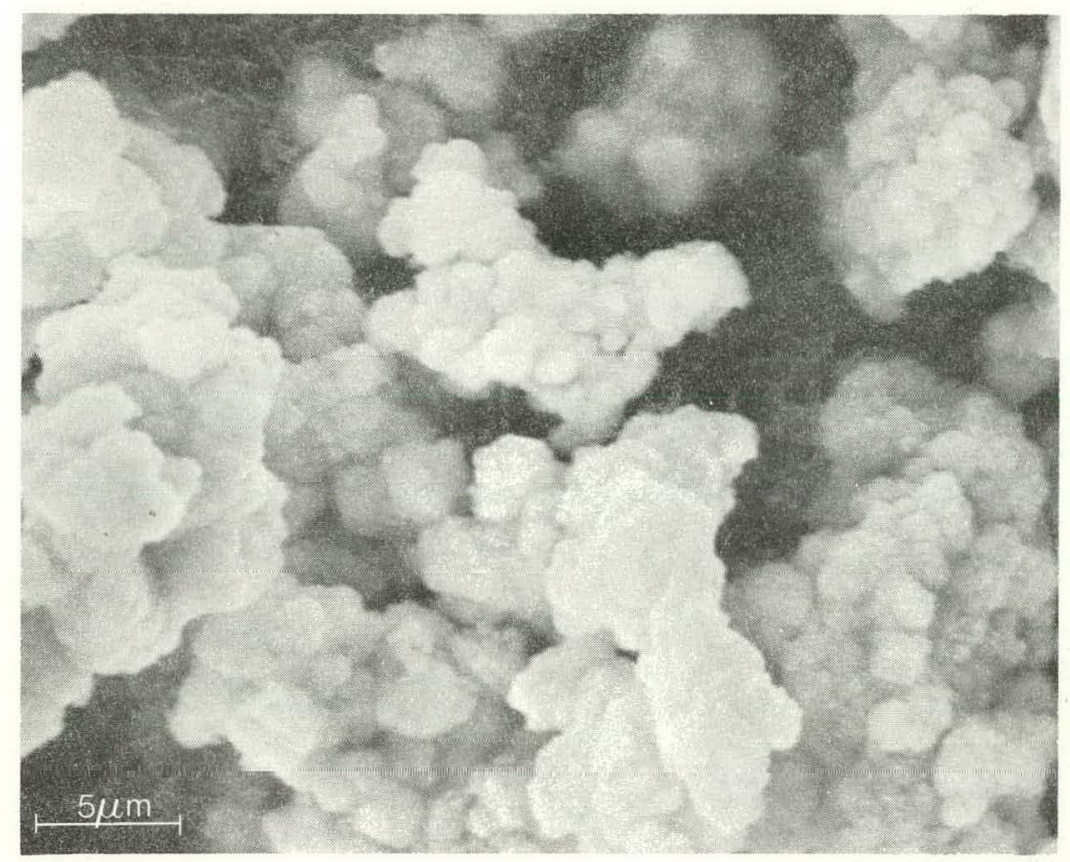

Figure 18. SEM micrograph $(3,000 \mathrm{X})$ of APU from run number 15 $(\mathrm{U}=0.200$ molar $, \mathrm{pH}=4.60, \mathrm{RFMR}=2.18)$.

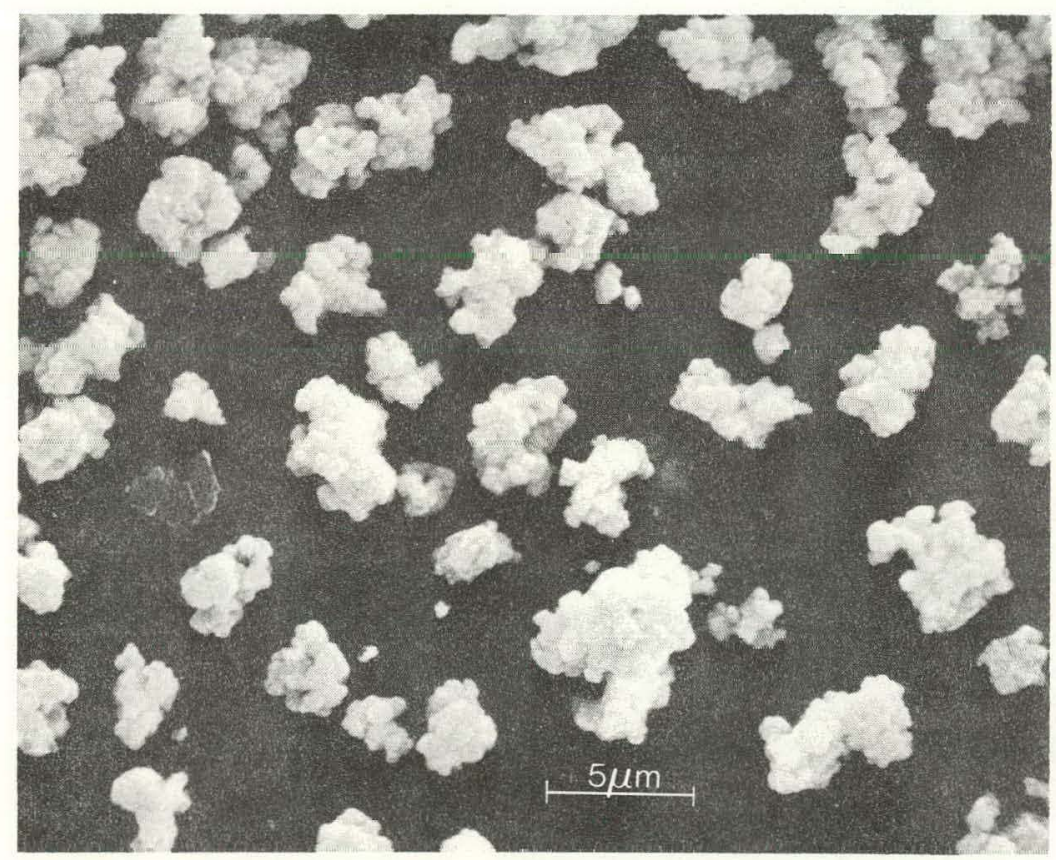

Figure 19. SEM micrograph $(3,000 \mathrm{X})$ of APU from run number 16 $(\mathrm{U}=0.0025$ molar $, \mathrm{pH}=5.47, \mathrm{RFMR}=2.19)$. 
Correlation of SEM Results with those of the Coulter counter

On a few of the SEM samples it was possible to detect several distinct large particles which were postulated to be agglomerates, and also, at higher magnification, several smaller particles which were postulated to be unagglomerated clusters. For two such samples (runs number 7 and 13) electron micrographs of both types of particles were used to obtain a particle size distribution and therefore an effective particle growth rate for each type of particle. These results are listed in Table 4 where they are compared with the growth rates obtained from the coulter counter analyses. The agreement between the SEM and Coulter counter results shows that the particles observed on the SEM micrographs are the clusters and agglomerates that were postulated from the coulter counter PDDs.

In reference to the particle counts obtained from the electron micrographs, the phrase 'particle size distribution' was used rather than 'particle density distribution' which was used in reference to the coulter counter results. The reason for this is to emphasize that a measurement of the original reactor volume (i.e. the suspension density) was not carried through to the electron microscope samples as it was to the Coulter counter samples. For this reason the particle density, and therefore the particle nucleate density, cannot be obtained from the micrographs. However, 
Table 4. Comparison of particle growth rates obtained from SEM micrographs and Coulter counter analyses.

\begin{tabular}{cccc}
$\begin{array}{c}\text { Run } \\
\text { Number }\end{array}$ & $\begin{array}{c}\text { Type of } \\
\text { Particles }\end{array}$ & SEM & $\begin{array}{c}\mathrm{G}_{\mathrm{e}} \times 10^{4}(\mu \mathrm{m} / \mathrm{s}) \\
\text { Coulter counter }\end{array}$ \\
\hline 7 & $\mathrm{c}$ & 2.21 & 4.23 \\
7 & $\mathrm{a}$ & 44.6 & 38.7 \\
13 & $\mathrm{c}$ & 3.89 & 3.96 \\
13 & $\mathrm{a}$ & 41.9 & 38.9 \\
\hline
\end{tabular}


the slope of the particle size distribution is not effected by the suspension density, and the effective particle growth rate can be obtained directly from the micrographs. This reasoning also applies to the TEM analyses which will be discussed later.

TEM Characterization of Crystallites

Due to the resolution limit of the scanning electron microscope the APU crystallites were further examined by the methods of transmission electron microscopy. Typical micrographs of the samples prepared for the TEM are shown in subsequent figures. Figures 20 and 21 show an even layer of APU crystallites from runs number 8 and number 10 respectively. The sizes of these crystallites can be easily measured and divided into a particle size distribution according to the characteristic length of area equivalent diameter. From this size distribution an effective growth rate of the characteristic length of the crystallites can be obtained. However, in order to obtain consistently reliable values for the crystallite growth rates the TEM samples must be prepared and studied in a carefully determined manner.

Since the TEM samples were prepared by first treating the dry APU powder with ultrasonics in water to redisperse the individual crystallites and then centrifuging to remove the redispersed clusters and agglomerates, the effects of 


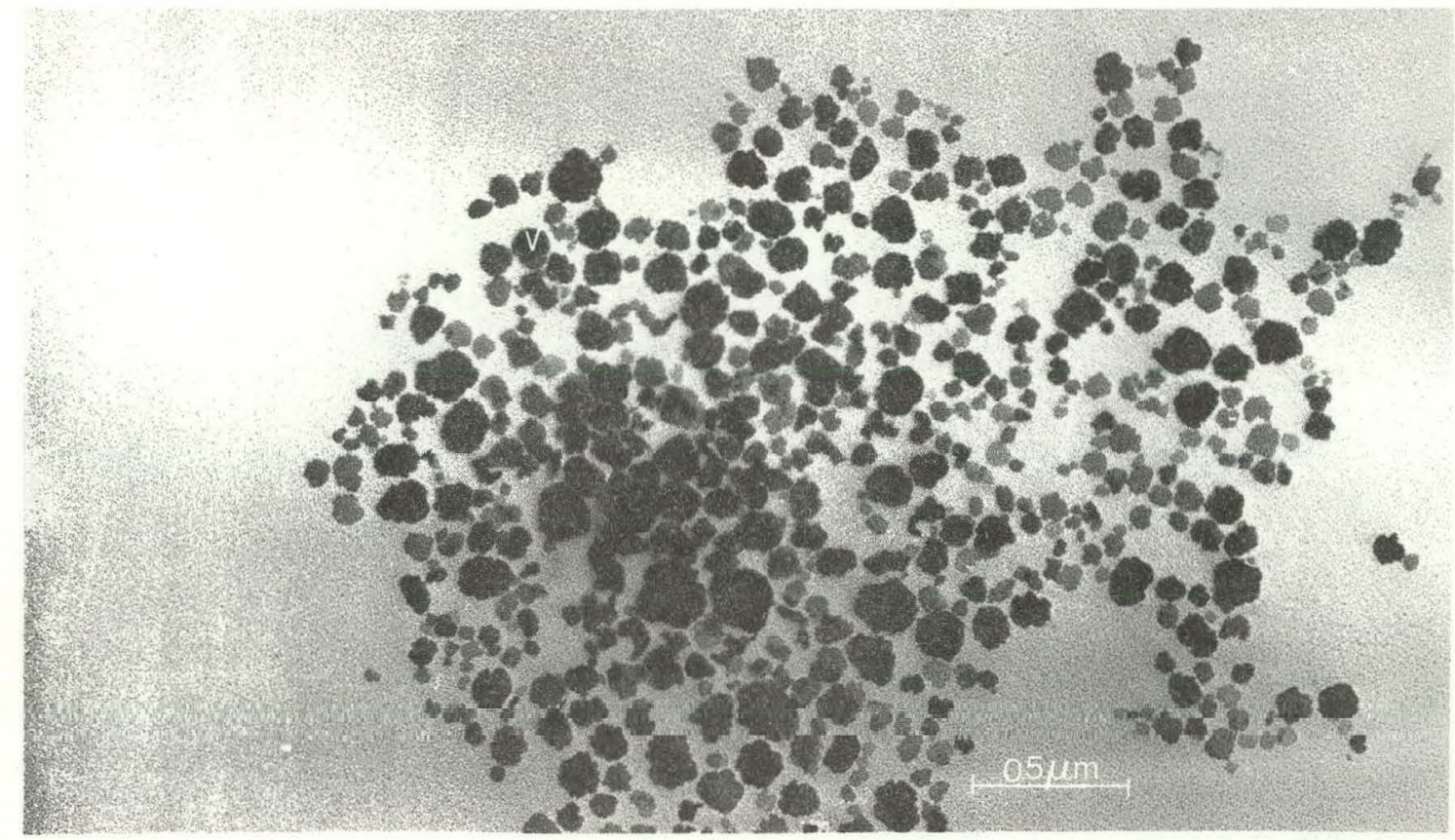

Figure 20. TEM micrograph $(32,000 \mathrm{X})$ of APU crystallites from run number 8 .

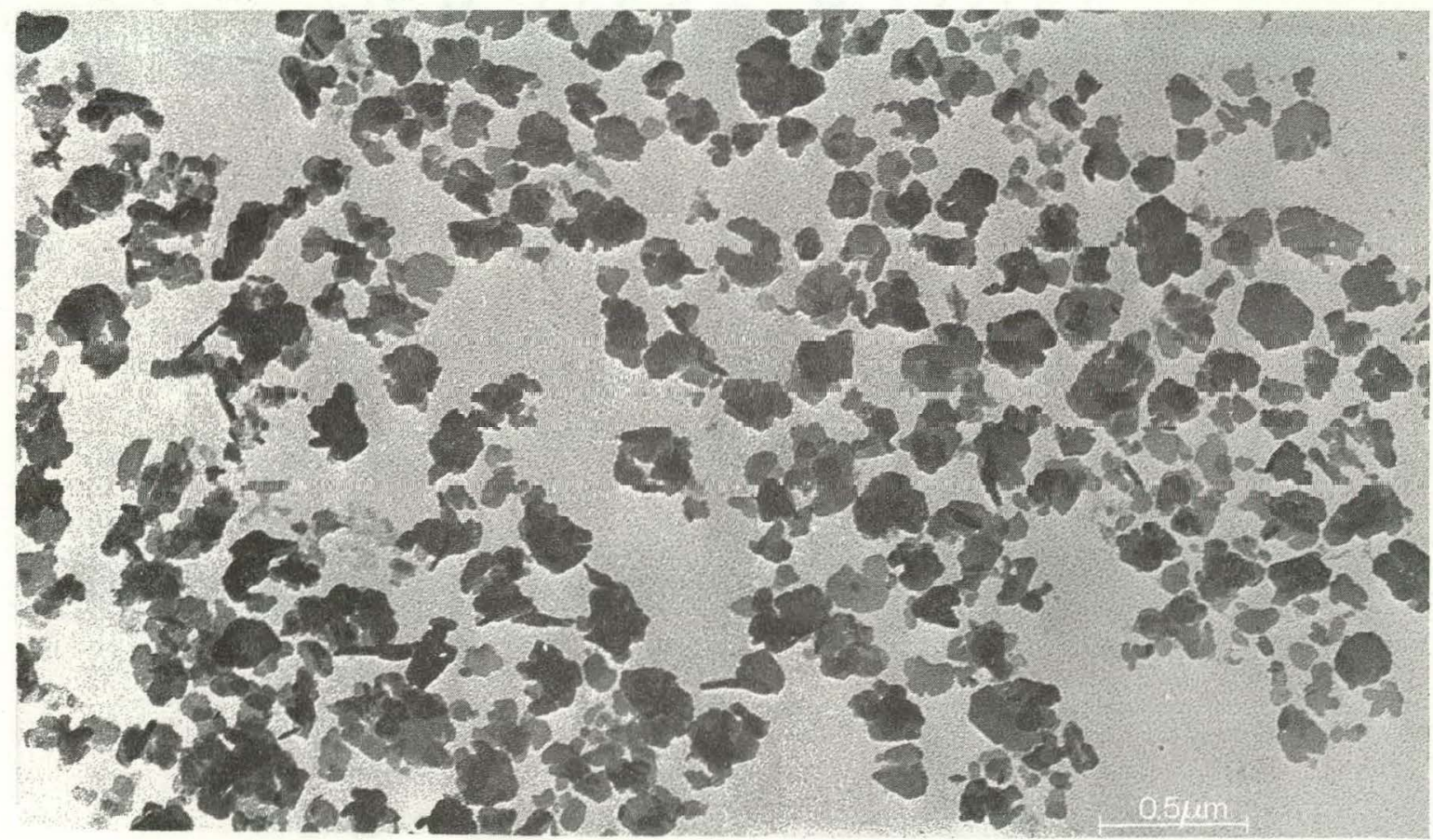

Figure 21. TEM micrograph $(32,000 \mathrm{X})$ of APU crystallites from run number 10 . 
varying degrees of ultrasonics and centrifuging on the measured crystallite growth rates were studied.

The effect of the ultrasonic treatment was studied by varying the length of treatment for several samples from the same experimental run and centrifuging each for six minutes before evaporating a drop of the resulting crystallite dispersion on a TEM sample grid. The growth rates which were measured from these samples are recorded in Figure 22. The point recorded for time equal to zero is from a sample which was dispersed by vigorous shaking but no ultrasonics.

It is observed that the measured growth rate initially drops very rapidly with time of ultrasonic treatment, but levels off to a constant value after about 30 minutes of treatment. This indicates that the first few minutes of ultrasonics produces a crystallite suspension which is classified towards the larger sizes. This phenomenon can be explained by the fact that the original suspension is unsaturated in the uranate and as the first several crystallites are dispersed the smaller sizes are dissolved. Once the solution becomes saturated with uranate the particle size distribution within the suspension, and therefore the measured growth rate, will level off to the true value. The shape of the curve in Figure 2.2 indicates that: (i) since the data points lie on such a smooth curve consistent and reliable growth rate values can be obtained by counting 


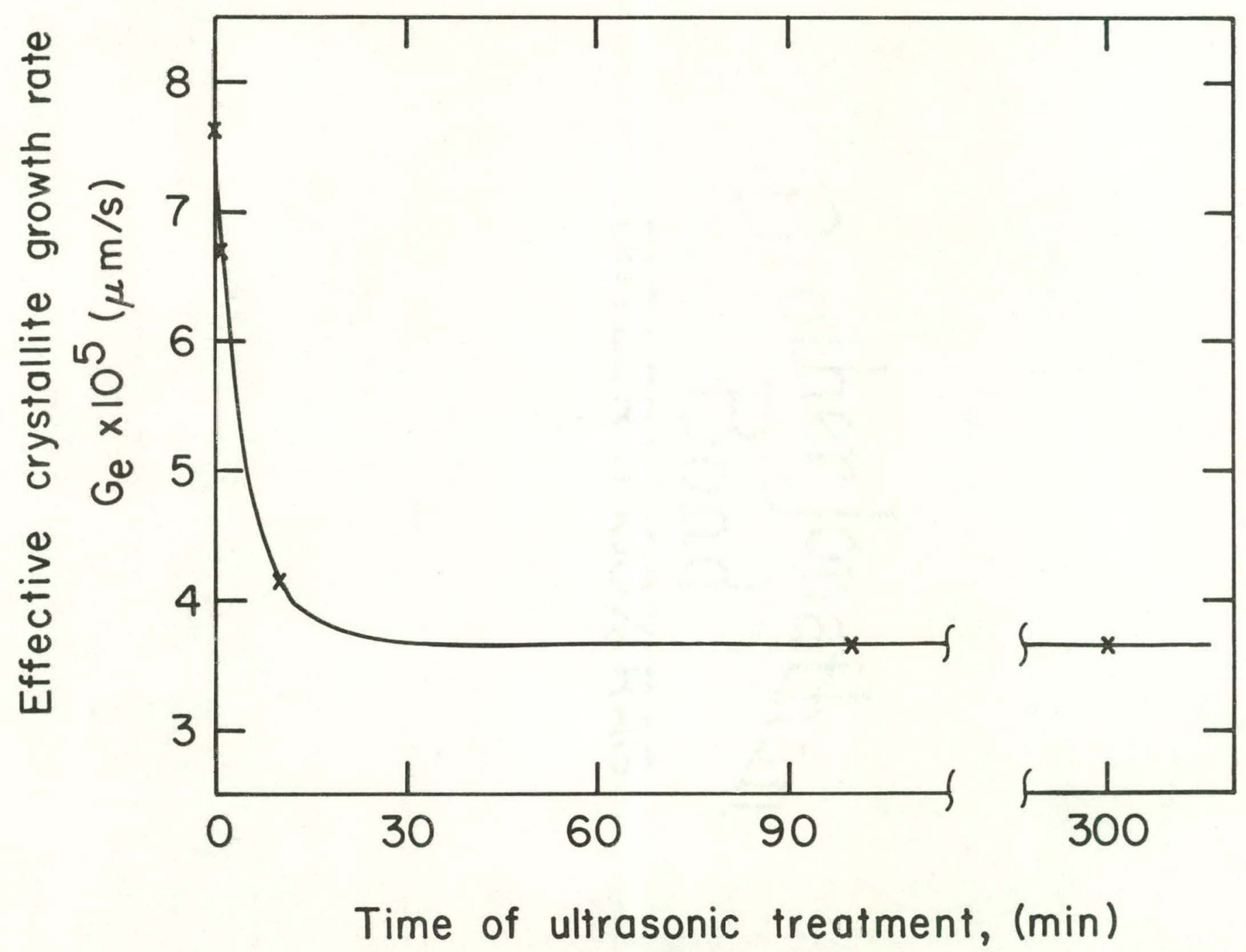

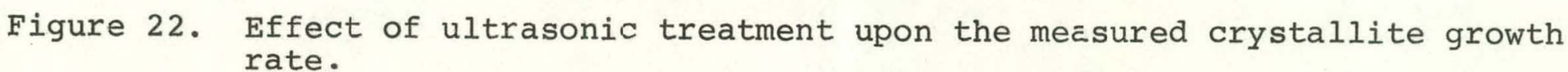


particle sizes from TEM micrographs; (ii) these consistent and reliable values can be obtained with ultrasonic treatment for a minimum of 30 minutes.

The effect of centrifuging was observed by treating with ultrasonics several samples from the same experimental run for 5 minutes and centrifuging the resulting suspensions for varying lengths of time before preparing the TEM samples. The values for the growth rate which were obtained appeared scattered, probably due to the fact that the 5 minute ultrasonic treatment does not approach the asymptotic growth rate value observed earlier. However, several important observations were still made. In the absence of centrifuging, even with gravity settling for over 24 hours, the suspension was not sufficiently cleared of clusters and agglomerates to produce a sample from which the individual crystallites were sufficiently characterizable. Centrifuging at a very high speed for several minutes can classify the crystallite suspension to a very significant extent due to the faster settling rates of the larger crystallites. Therefore, the centrifuge treatment must be optimized to a point where the suspension is sufficiently cleared of clusters and agglomerates but yet not significantly classified according to crystallile sizes.

Two of the micrographs used in this work for measuring crystallite size distributions are shown in Figures 23 and 


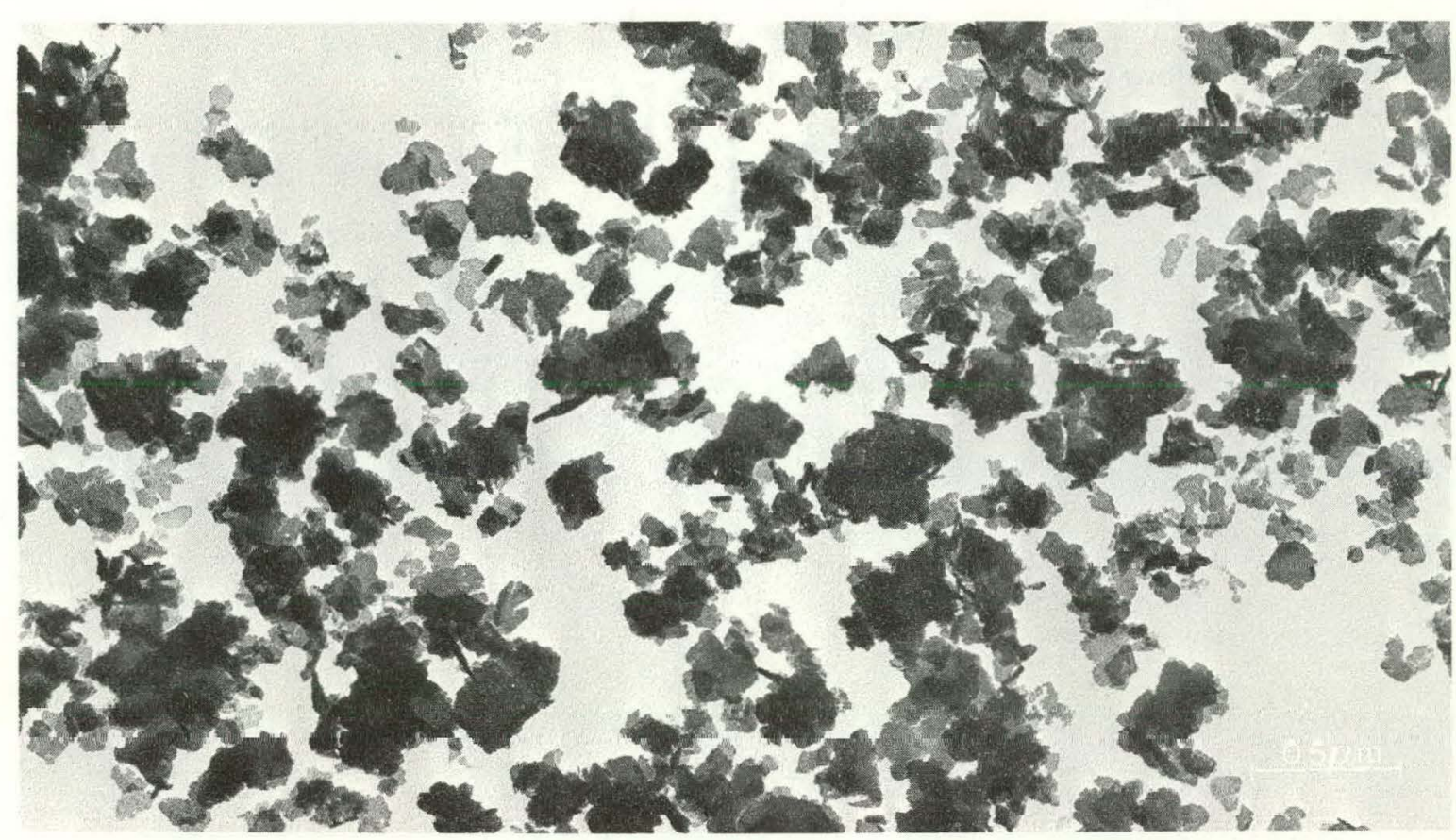

Figure 23. TEM micrograph $(32,000 \mathrm{x})$ of APU crystallites from run number 9 .

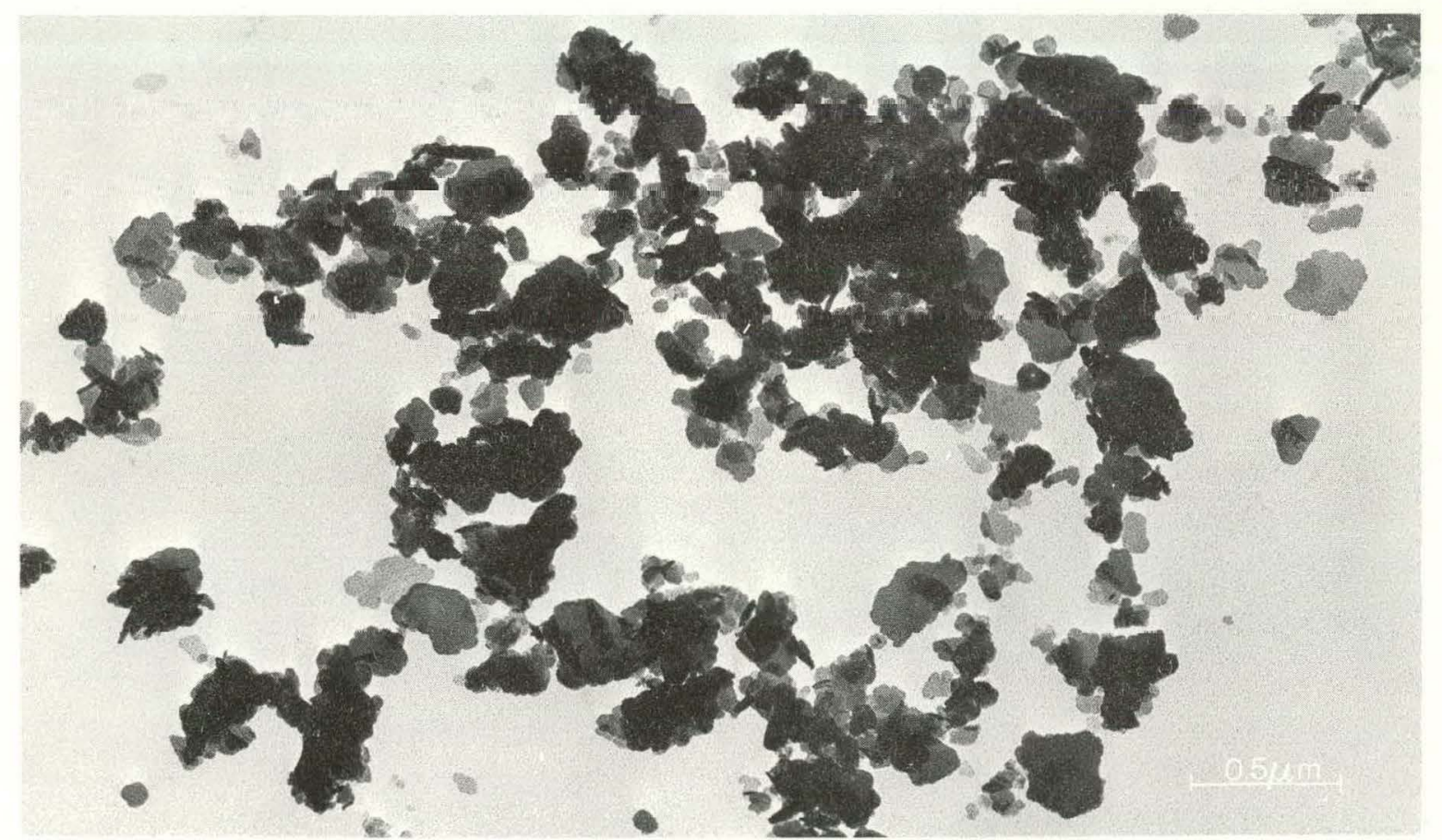

Figure 24. TEM micrograph $(32,000 \mathrm{X})$ of APU crystallites from run number 13. 
24. Even though several clusters can be observed in each of these pictures, enough individual crystallites are. distinguishable to obtain a particle size distribution. It should be observed from these pictures that since the TEM images result from a transmitted electron beam even crystallites that overlap one another have distinguishable edges and can be measured.

Each of the particle size distributions which were obtained from the TEM micrographs were fitted to a straight line of log of number density vs. particle size, and the effective crystallite growth rates were obtained from the slopes. A plot of a crystallite size distribution for run number 13 is given in Figure 25. For most samples it was found that the particle size distribution drops off from Iinear at the lower size ranges, presumably due to dissolution of the very fines. For this reason the smallest size range was excluded from the line fit if it appeared to deviate from linear.

The kinetic results obtained from the TEM micrographs are given in Table 5. Figure 26 is a plot of the measured effective crystallite growth rate vs. reacting $\mathrm{pH}$ for a constant uranium concentration of 0.0249 molar. Each point on the graph represents the average of two or three particle counts made for a given sample, the ranges of which are also indicated. With the exception of the value obtained for run 
Table 5. Crystallite growth rates from TEM analysis

\begin{tabular}{|c|c|c|c|c|}
\hline RFMR & $\begin{array}{l}\text { Run } \\
\text { Number }\end{array}$ & $\mathrm{G}_{e, \times 10^{5}} \times{ }_{(\mu \mathrm{m} / \mathrm{s})}$ & $(\operatorname{moles} / \ell)$ & \\
\hline 2.08 & 11 & 5.07 & 0.0249 & व \\
\hline 2.15 & 12 & 4.44 & 0.0249 & \\
\hline 2.17 & 13 & 4.72 & 0.0249 & \\
\hline 2.19 & 8 & 4.08 & 0.0249 & \\
\hline 2.20 & 7 & 4.06 & 0.0249 & \\
\hline 2.23 & 9 & 5.52 & 0.0249 & \\
\hline 2.28 & 10 & 4.20 & 0.0249 & \\
\hline 2.34 & 6 & 3.84 & 0.0249 & \\
\hline 2.58 & 2 & 4.48 & 0.0249 & \\
\hline 3.47 & 14 & 5.46 & 0.0249 & \\
\hline 3.48 & 3.8 & 5.29 & 0.2000 & \\
\hline 3.48 & 19 & 3.87 & 0.0100 & \\
\hline .3 .48 & 20 & 7.74 & 0.1000 & \\
\hline
\end{tabular}




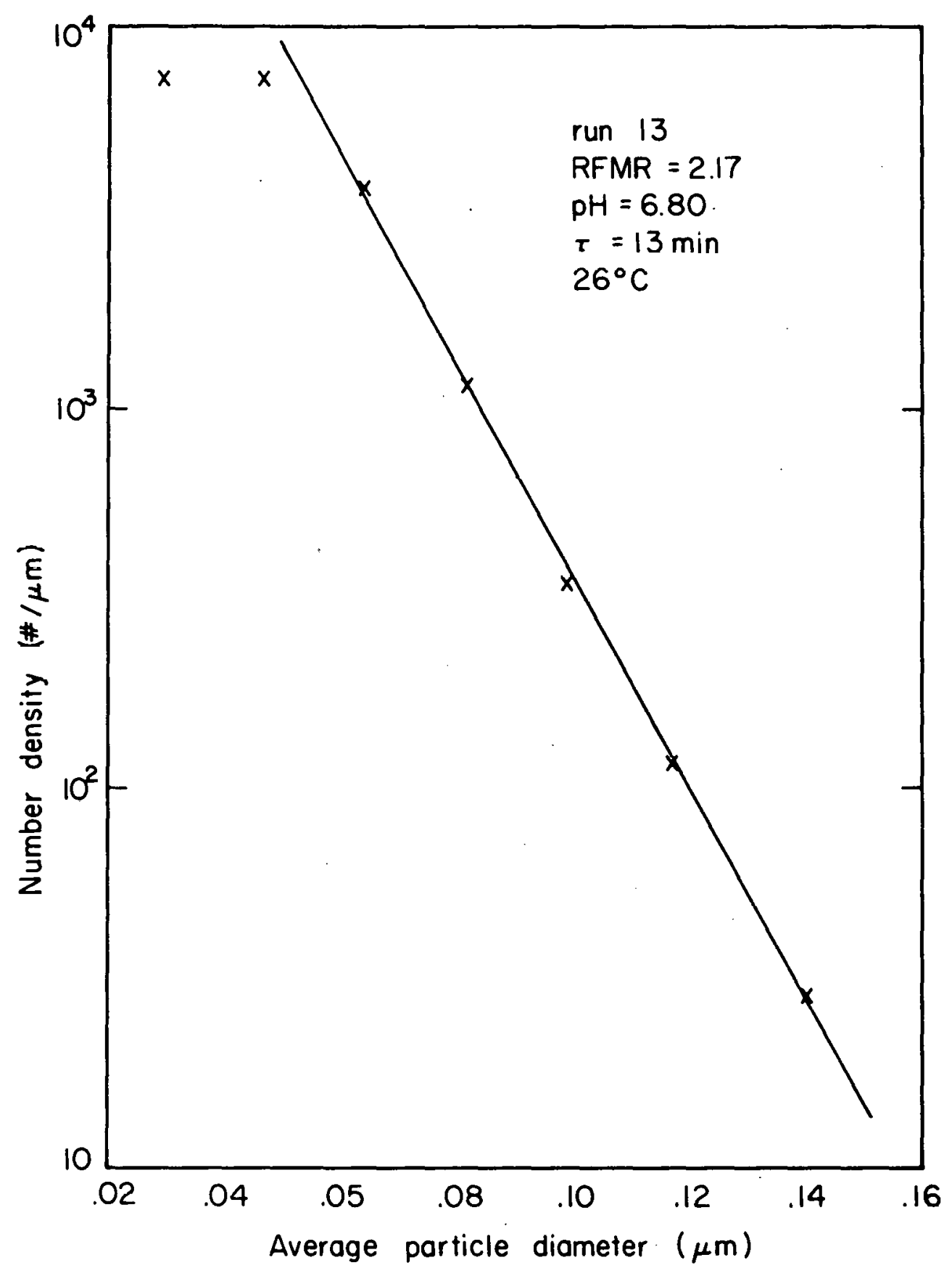

Figure 25. Crystallite size distribution from SEM micrograph. 


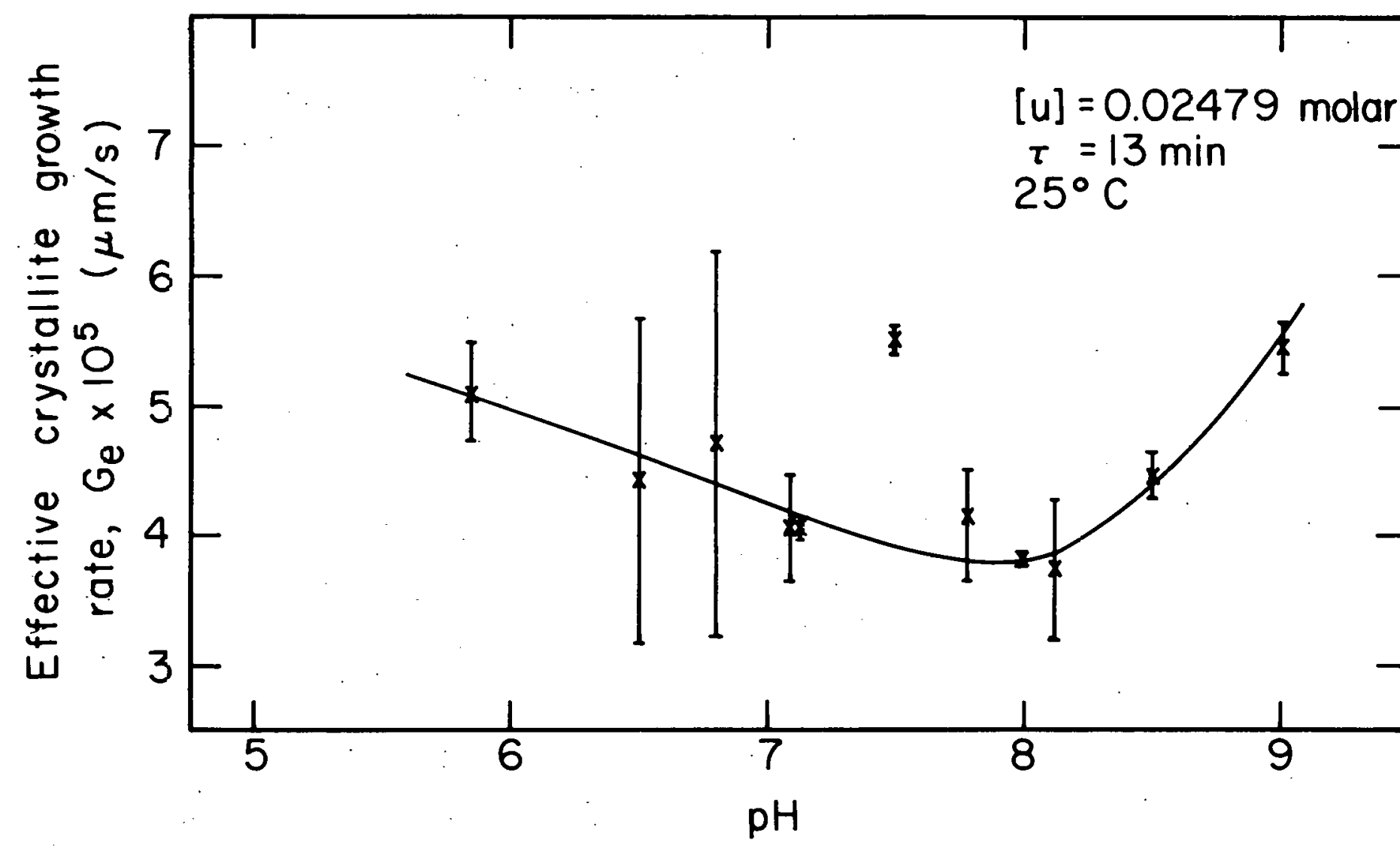

วี

Figure 26. Dependence of crystallite growth rate on the $\mathrm{pH}$ of precipitation 
number 9, a smooth curve can be drawn through the experimental points which goes through a minimum at a pH of about 8.0. Figure 27 is a graph of crystallite growth rate vs. RFMR for the same experimental runs and is seen to go through a minimum at a RFMR of about 2.35. Since the average crystallite size for a particular sample is proportional. to the effective growth rate, the crystallite sizes must go through the same minimum with $\mathrm{pH}$ and RFMR. The SEM pictures of runs number 11,6 , and 14, shown previously in Figures 11, 15 and 17 , confirm this trend.

This minimum in the crystallite growth rate is the result of the combination of coagulation rate changes and supersateration changes. As mentioned earlier, the clusters and agglomerates become more loosely coagulated as the $\mathrm{pH}$ or RFMR is increased, resulting in increased surface area being available for crystal growth. This phenomenon is the result of preferential ion absorption which produces an electrostatic potential gradient about each particle causing them to repel each other $(22,23)$. This effect is increased through the addition of ammonium hydroxide. The supersaturation, which determines the APU crystallite growth rate and nucleation rate, is decreased because of the increase in surface area. This effect is counteracted by the increase in supersaturation which arises from the extreme decrease in APU solubility as the $\mathrm{pH}$ increases (29). The combination 


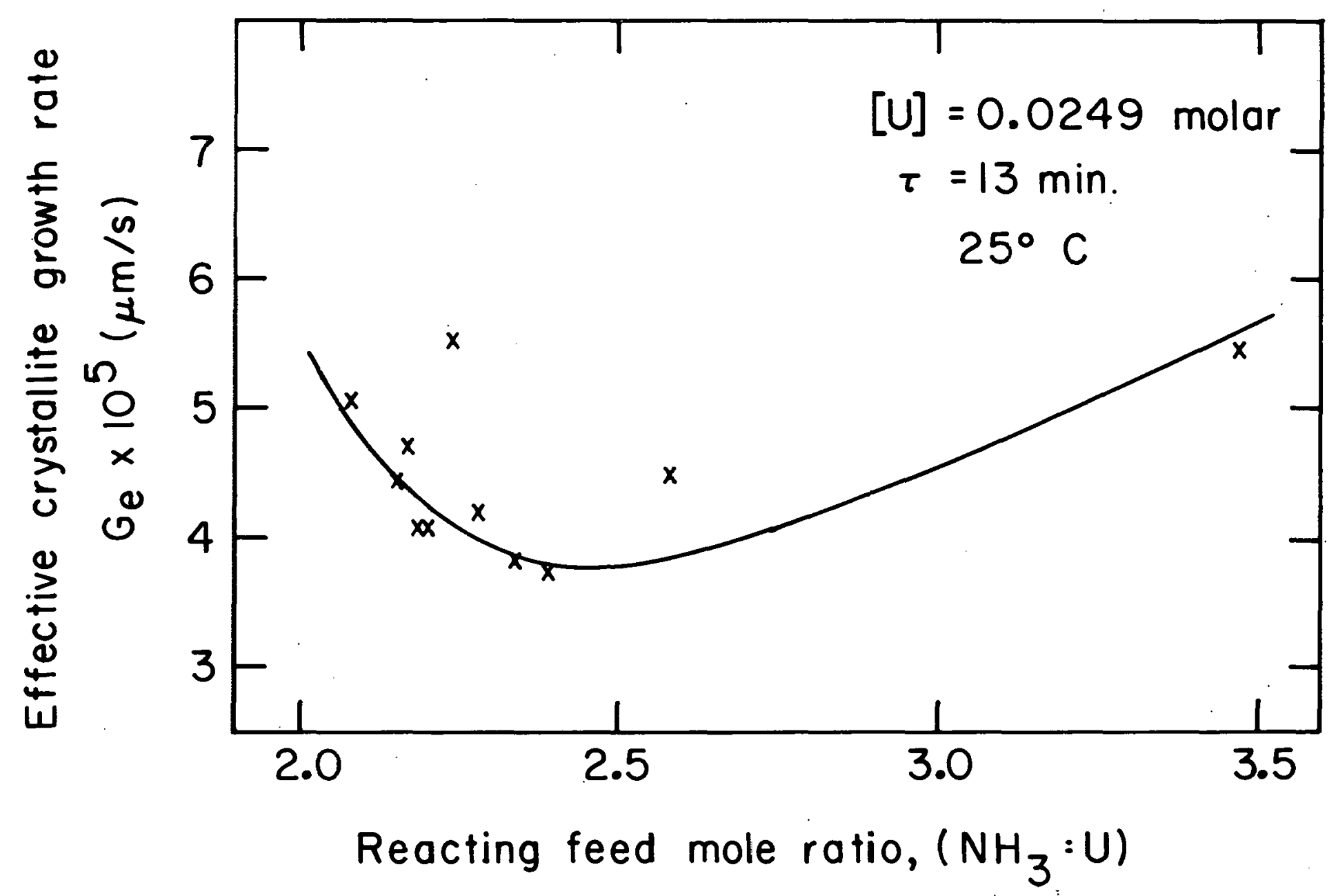

Figure 27. Dependence of crystallite growth rate on $\mathrm{NH}_{3}: U$ reacting feed mole
ratio. 
of these supersaturation effects results in a minimum in the crystallite growth rate.

These same effects also influence the agglomerate and cluster growth rates. Figure 28 shows the crystallite, cluster, and agglomerate growth rates as a function of $\mathrm{pH}$. Figure 29 shows the effect of the RFMR on these growth rates. The changes in the supersaturation and coagulation effects with $\mathrm{pH}$ results in a maximum in both the cluster and agglomerate growth rates. However, since the clusters involve fewer crystallites this trend is nut as nuliceable for them.

In order to calculate the surface area per unit volume of suspension a surface area shape factor must be obtained for each type of particle. For the clusters and agglomerates it will be assumed that all the particles are spherical with negligible open pore volume. Since the Coulter counter measurements use the spherical volume equivalent diameter as the characteristic length of the particles, the surface area shape factor for the clusters and agglomerates can be calculated by

$$
4 \pi\left(\frac{L}{2}\right)^{2}=k_{a} L^{2}
$$

or

$$
\mathrm{k}_{\mathrm{a}, \mathrm{c}}=\mathrm{k}_{\mathrm{a}, \mathrm{a}}=\pi
$$




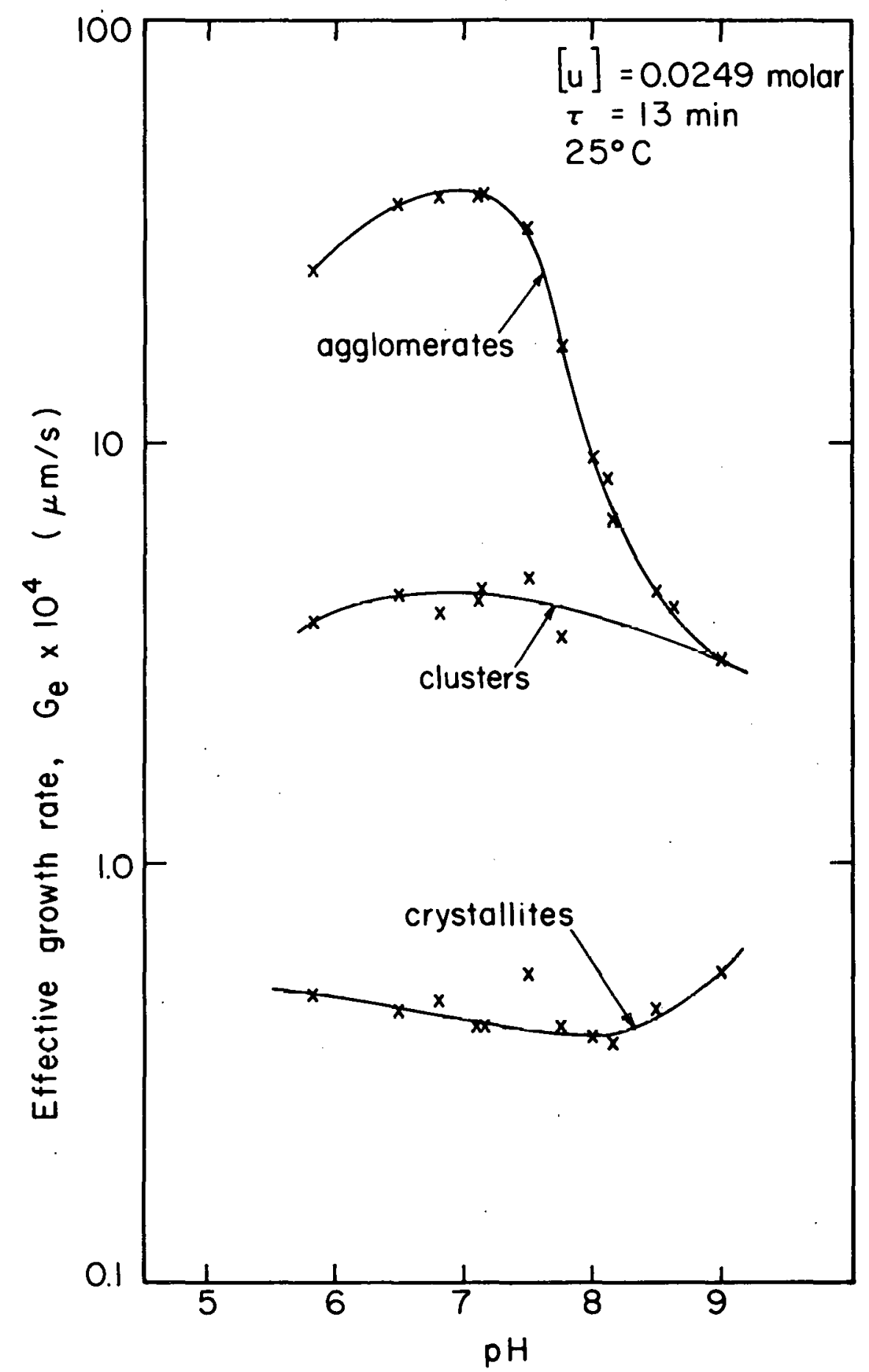

Figure 28. Dependence of agglomerate, cluster, and crystallite growth rates on $\mathrm{pH}$. 


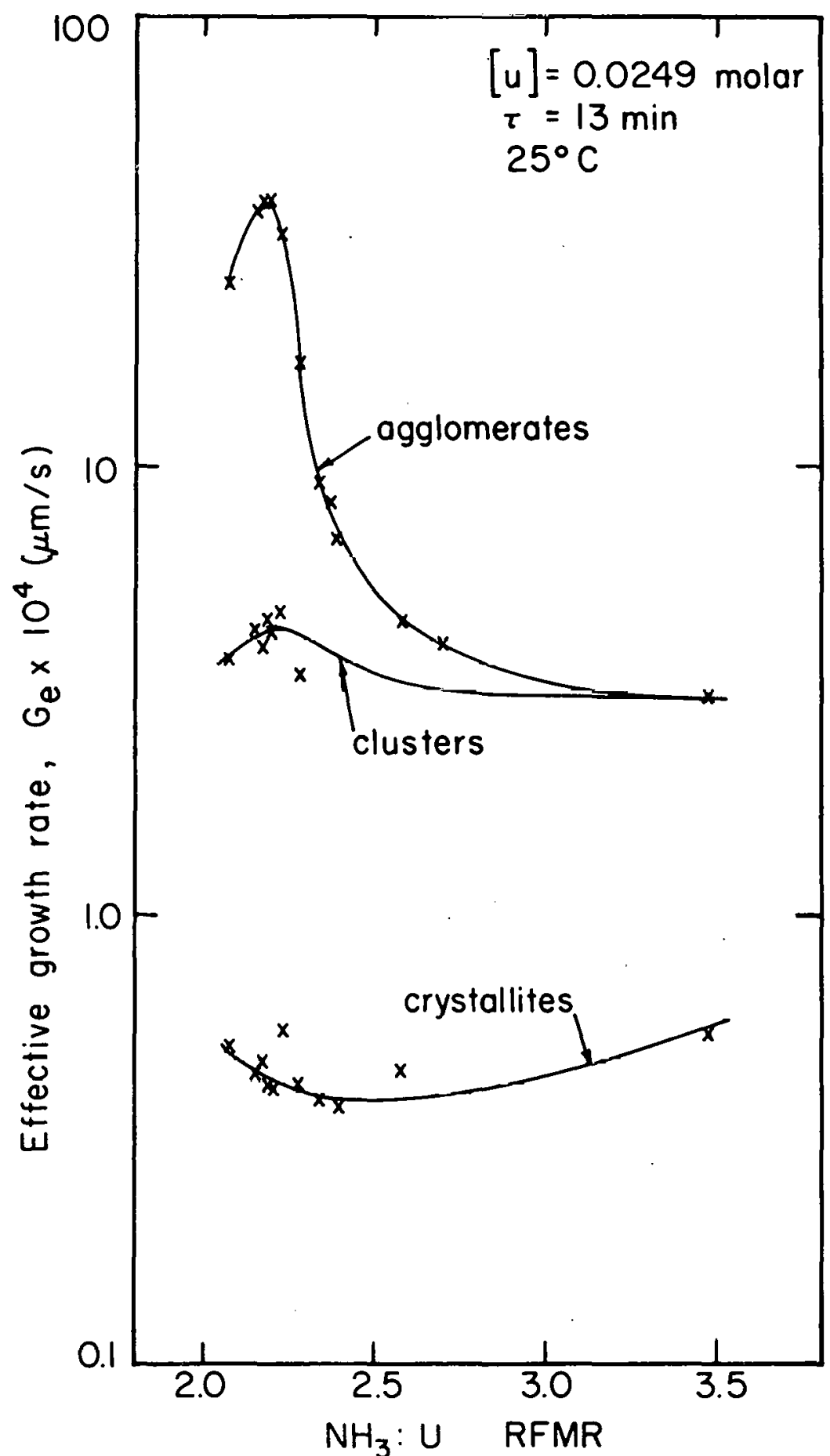

Figure 29. Dependence of agglomerate, cluster, and crystallite growth rates on $\mathrm{NH}_{3}: \mathrm{U}$ reacting feed mole ratio. 
Since the crystallites are observed to be very thin platelets it will be assumed that the surface area of the edge of the crystal is negligible in comparison to the two faces. Then for a characteristic dimension of the circular area equivalent diameter of the face of the crystal the surface area shape factor is found to be

$$
2 \pi\left(\frac{L}{2}\right)^{2}=k_{a, x} L^{2}
$$

or

$$
\mathrm{k}_{\mathrm{a}, \mathrm{x}}=\pi / 2
$$

Similarly, calculation of the suspension density requires a value for a volumetric shape factor for each type of particle. Following the reasoning outlined in the previous paragraph a cluster and agglomerate volumetric shape factor is calculated as

$$
4 / 3 \pi\left(\frac{L}{2}\right)^{3}=k v^{3}
$$

or

$$
\mathrm{k}_{\mathrm{v}, \mathrm{c}}=\mathrm{k}_{\mathrm{v}, \mathrm{a}}=\pi / 6
$$

In order to obtain the crystallite volumetric shape factors it is necessary to measure an average ratio of crystallite thickness to characteristic length (W/L) 
for the crystallites of the APU powder samples. The volumetric shape factor for each powder is then calculated by

$$
\pi\left(\frac{L}{2}\right)^{2} w=k_{v, x^{L}}{ }^{3}
$$

or

$$
\mathrm{k}_{\mathrm{v}, \mathrm{x}}=\pi / 4(\mathrm{~W} / \mathrm{L})
$$

The $\mathrm{W} / \mathrm{L}$ ratios for the crystallites were obtained by two different methods. It can be seen in Figures 23 and 24 that several crystallites are standing on edge. This makes it possible to measure the $\mathrm{W} / \mathrm{L}$ ratios directly. Several of the TEM samples were also shadowed with germanium metal at a $15^{\circ}$ angle and re-examined in the microscope. An example of such a sample is shown in Figure 30 . The light shadow appearing next to each crystallite is that area which was not coated with the metal because it was hidden by the crystallite from the metal source. From the length of the shadows the thicknesses of the crystallites and therefore an average shape factor can be determined. The coarse looking background in Figure 30 is due to the roughness of the carbon film on which the APU particles are suspended. The resulting $W / L$ values for the crystallites showed no correlation to the reacting conditions and an 


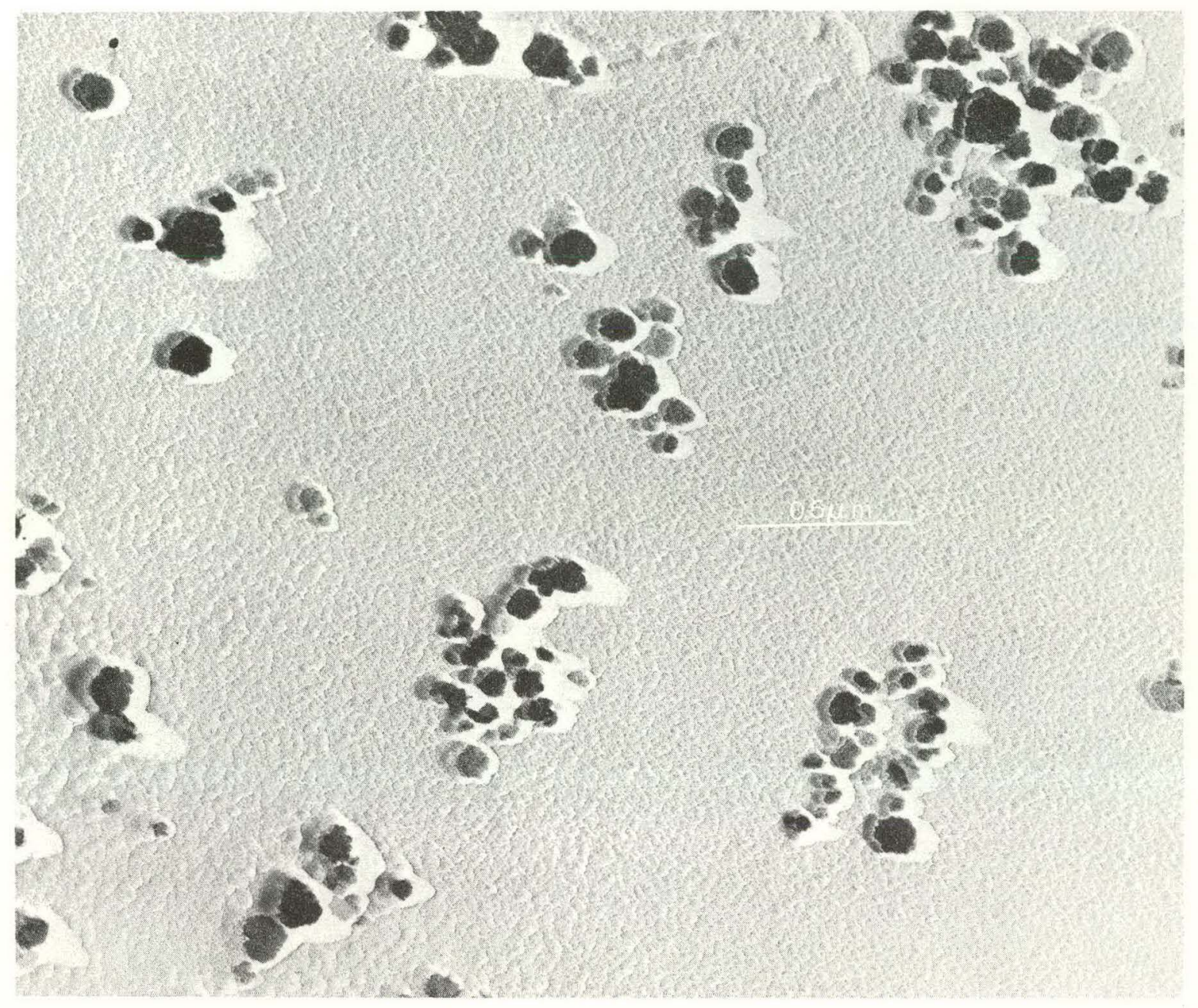

Figure 30. TEM micrograph $(44,000 \mathrm{X})$ of APU crystallites shadowed with germanium metal. 
average value for all of the runs of $W / L=0.115$ was used to obtain a crystallite volumetric shape factor from Equation (63b) of $k_{v, x}=0.09$

\section{Calculation of Kinetic Parameters}

The experimental values obtained in this work for the crystallite growth rates and crystallite shape factors are the only values that were missing in Hoyt's experimental analysis of the APU precipitation process. Using the kinetic theory of precipitation outlined earlier, values for all of the kinetic parameters can now be determined.

Some of the kinetic parameters obtained for run number 7 are listed in the third column of Table 6 . The second column lists the equation used to calculate the respective parameter. A literature value (8) for the crystallite density of $\rho_{X}=4.97 \mathrm{~g} / \mathrm{cm}^{3}$ was used for all particle densities in obtaining the suspension densities for each type of particle. This density value is supported by an experimentally obtained powder density of $5.18 \mathrm{~g} / \mathrm{cm}^{3}$. However, since sample sizes of only approximately $100 \mathrm{mg}$ were used for the experimental density measurements (volume displacement method) the literature value was assumed to be more accurate. 
Table 6. Parameters for run number 7

\begin{tabular}{|c|c|c|c|}
\hline Parameter & $\begin{array}{l}\text { Equation } \\
\text { used }\end{array}$ & $\begin{array}{c}\text { Calculated } \\
\text { values }\end{array}$ & $\begin{array}{l}\text { Assuming } \\
\overline{\mathrm{L}}_{\mathrm{m}}=0\end{array}$ \\
\hline$E_{x}(\mu m)$ & 21 & $3.17 \times 10^{-2}$ & $3.17 \times 10^{-2}$ \\
\hline $\bar{L}_{c}(\mu \mathrm{m})$ & 23 & 0.352 & 0.330 \\
\hline $\bar{L}_{a}(\mu \mathrm{m})$ & 32 & 3.46 & 3.02 \\
\hline$\overline{\mathrm{L}}_{\mathrm{m}, \mathrm{c}}(\mu \mathrm{m})$ & 20 & $2.22 \times 10^{-2}$ & 0 \\
\hline$\overline{\mathrm{L}}_{\mathrm{m}, \mathrm{a}}(\mu \mathrm{m})$ & 22 & 0.444 & 0 \\
\hline $\mathrm{L}_{\mathrm{d}, \mathrm{x}}(\mu \mathrm{m})$ & 43 & $9.51 \times 10^{-2}$ & $9.51 \times 10^{-2}$ \\
\hline${ }^{\mathrm{L}} \mathrm{d}, \mathrm{c}^{(\mu \mathrm{m})}$ & 44 & 1.01 & 0.990 \\
\hline$L_{d, a}(\mu m)$ & 45 & 9.50 & 9.06 \\
\hline$\overline{\mathrm{n}}_{\mathrm{c}}^{\mathrm{O}}\left(\# / \mu \mathrm{m} \cdot \mathrm{cm}^{3}\right)$ & 24 & $6.21 \times 10^{8}$ & $6.64 \times 10^{8}$ \\
\hline $\overrightarrow{\mathrm{n}}_{\mathrm{a}}^{0}\left(\# / \mu \mathrm{m} \cdot \mathrm{cm}^{3}\right)$ & 25 & $6.67 \times 10^{6}$ & $7.73 \times 10^{6}$ \\
\hline$A_{c}\left(\mathrm{~cm}^{2} / \mathrm{cm}^{3}\right)$ & 57 & 1.50 & 1.50 \\
\hline$A_{a}\left(\mathrm{~cm}^{2} / \mathrm{cm}^{3}\right)$ & 57 & 13.4 & 13.4 \\
\hline$M_{C}\left(g / \mathrm{cm}^{3}\right)$ & 35 & $1.23 \times 10^{-4}$ & $1.23 \times 10^{-4}$ \\
\hline$M_{a}\left(g / \mathrm{cm}^{3}\right)$ & 36 & $1.00 \times 10^{-2}$ & $1.00 \times 10^{-2}$ \\
\hline
\end{tabular}


By.assuming that the mass fraction of the agglomerates in run number 11 is 1.0 (see Figure 32) a total suspension density of $\mathrm{M}_{\mathrm{T}}=1.98 \times 10^{-2} \mathrm{~g} / \mathrm{cm}^{3}$ can be obtained from Equations (36) and (37). If $M_{T}$ is assumed to be equivalent for all experimental runs at the same uranium concentration, the crystallite suspension density for run number 7 can be calculated from Equation (58) and the values given in Table 6 to be $0.97 \mathrm{~g} / \mathrm{cm}$. By use of Equation (34) a value forthe nucleate suspension density for the crystallites of

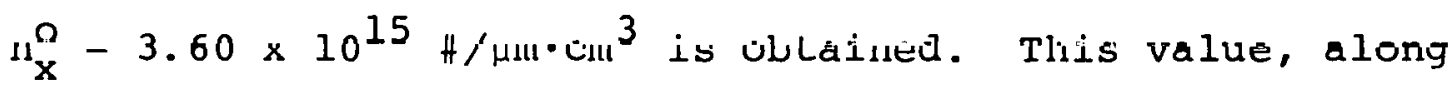
with the crystallite growth rate, makes it possible to add the crystallite population density distribution to the distributions for the clusters and agglomerates which are given in Figure 8. All three population density distributions are shown in Figure 31 .

Equation (55) can now be used to obtain a value for the average crystallite growth rate of $\mathrm{G}_{\mathrm{X}}^{\mathrm{av}}=2.84 \times 10^{-5} \mu \mathrm{m} / \mathrm{s}$. This is the average growth rate over the entire surface of the crystallites. In order to obtain the growth rate of the characteristic dimension of the crystallites the geometry of the crystalilite must be considered;

Volumetric growth rate $=$ (surface area) $\mathrm{X}$ (average linear

$$
\text { growth rate })=k_{a, x}{ }^{2} G_{x}^{a v}=L W G_{x}+\left(\frac{L}{2}\right) \underset{L}{L} G_{x}
$$

where $G_{x}$ is the growth rate of the characteristic dimension. 


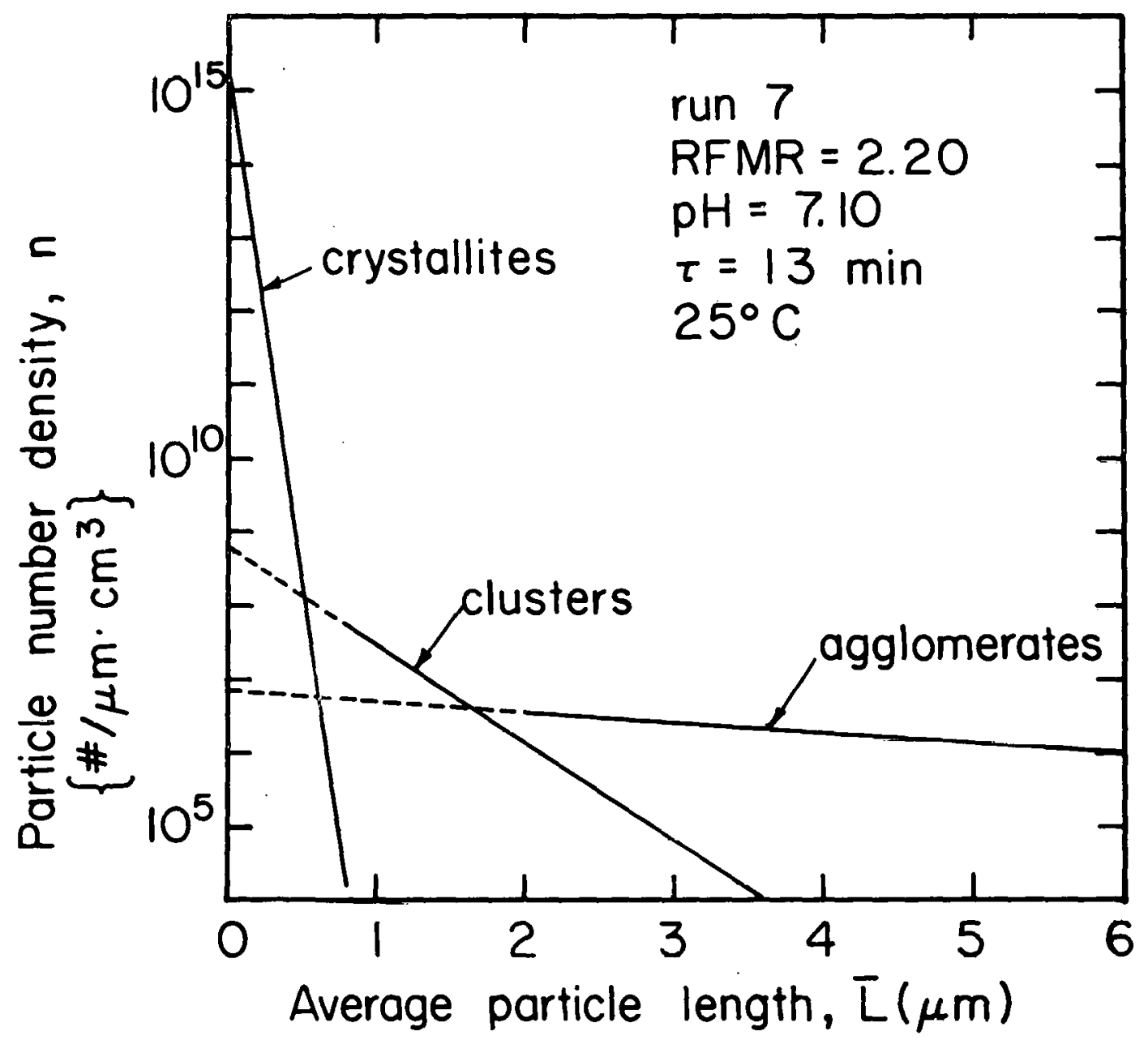

Figure 31. Population density distributions for run 7 . 
Equation (59) simplifies to

$$
G_{x}=\frac{1}{5} \frac{k_{a, x}}{k_{v, x} G_{x}^{a v}}=9.91 \times 10^{-5} \mu \mathrm{m} / \mathrm{s}
$$

Now equations (10), (11), (14), (15), (16) and (19) can be solved for the 6 parameters,

$$
\begin{aligned}
& G_{c}=6.09 \times 10^{-2} \mu \mathrm{m} / \mathrm{s} \\
& G_{a}=6.48 \times 10^{-2} \mu \mathrm{m} / \mathrm{s} \\
& G_{a}^{\prime}=-6.09 \times 10^{2} \mu \mathrm{m} / \mathrm{s} \\
& k_{c}=0.1836 \mathrm{sec}^{-1} \\
& k_{x}^{\prime}=1.92 \mathrm{sec}^{-1} \\
& k_{x}^{\prime \prime}=-1.92 \mathrm{sec}^{-1}
\end{aligned}
$$

These parameter values do not look reasonable -particularly the negative value for $G_{a}^{\prime}$ which indicates that crystallites are breaking away from rather than coagulating with the agglomerates. The most likely reason for these apparently unreasonable numbers is the error that may be introduced in calculating the crystallite suspension density and nucleate population density by difference rather than obtaining it directly.

The fourth column in Table 6 shows the values that would be obtained for the parameters if it is assumed that 
the minimum sizes for the clusters and agglomerates is zero. This assumption would allow the calculation of the relevant kinetic parameters without information on the crystallite growth rates. It should be noted that values for the surface areas and suspension densities of the cluster and crystallites agree within three significant figures for the two methods of calculation.

The calculated kinetic parameters can now be used to make useful interpretations about the APU precipitation process. Perhaps the most important characteristic of a given powder is the total size distribution. This can be conveniently defined by a combination of the particle size distribution and the suspension density fraction for each of the three types of particles. By using Equations (9), (13) and (18) a size distribution for each type of particle can be found for any reacting condition within the range of the experimental conditions which have been investigated. From Equations (34) through (40) the suspension density fraction of each type of particle can be calculated for the same range of reacting conditions. Figures (32) through (35) are graphs of the suspension density fractions as a function of $\mathrm{pH}$ and RFMR. In this case the suspension densities of the clusters and agglomerates were calculated directly and the suspension density of the crystallites was obtained by. difference (Equation (58)). 


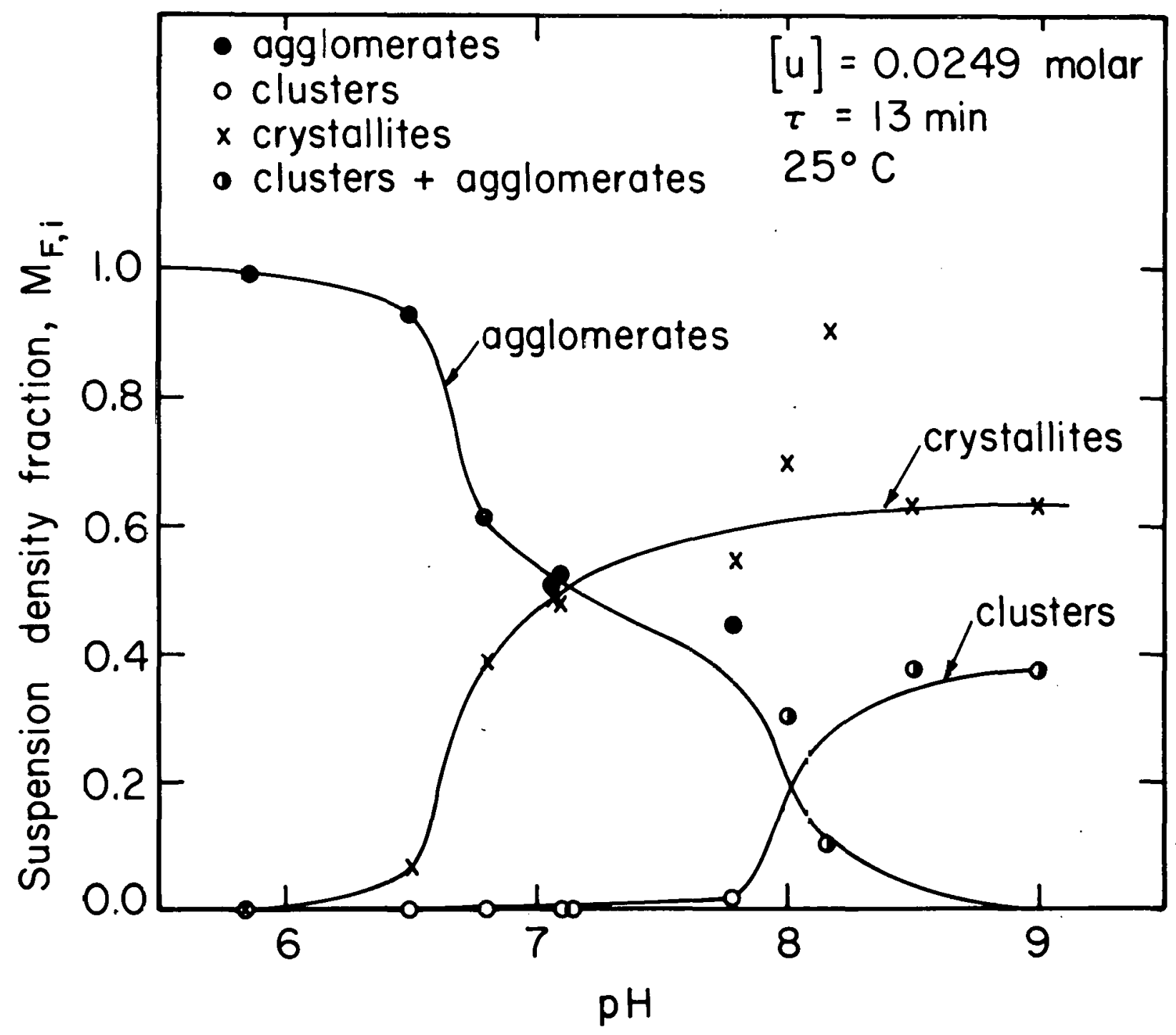

Figure 32. Suspension density fraction as a function of pH at a uranium concentration of 0.0249 . 


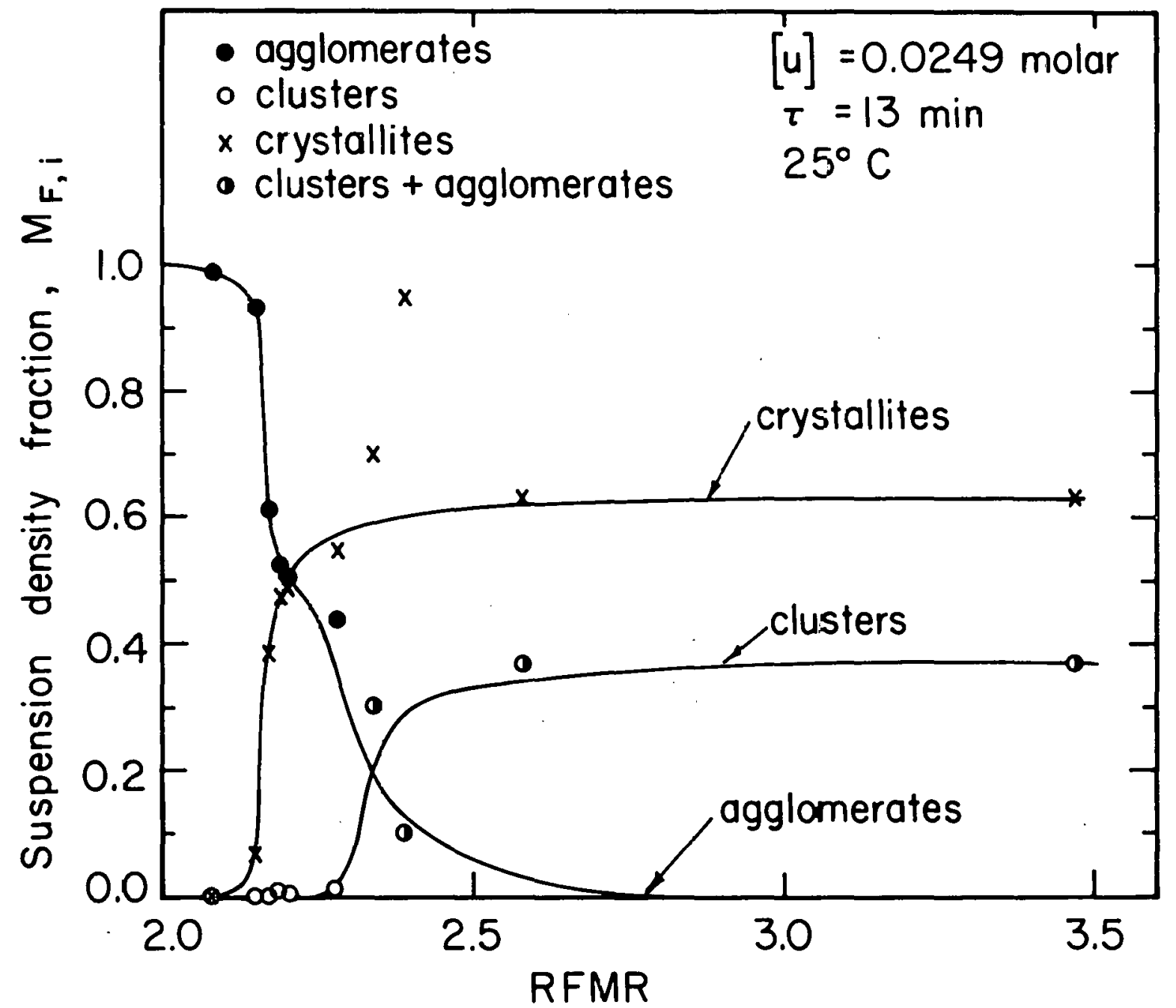

Figure 33: Suspension density fraction as a function of RFMR at a uranium con-
centration of 0.0249 . 


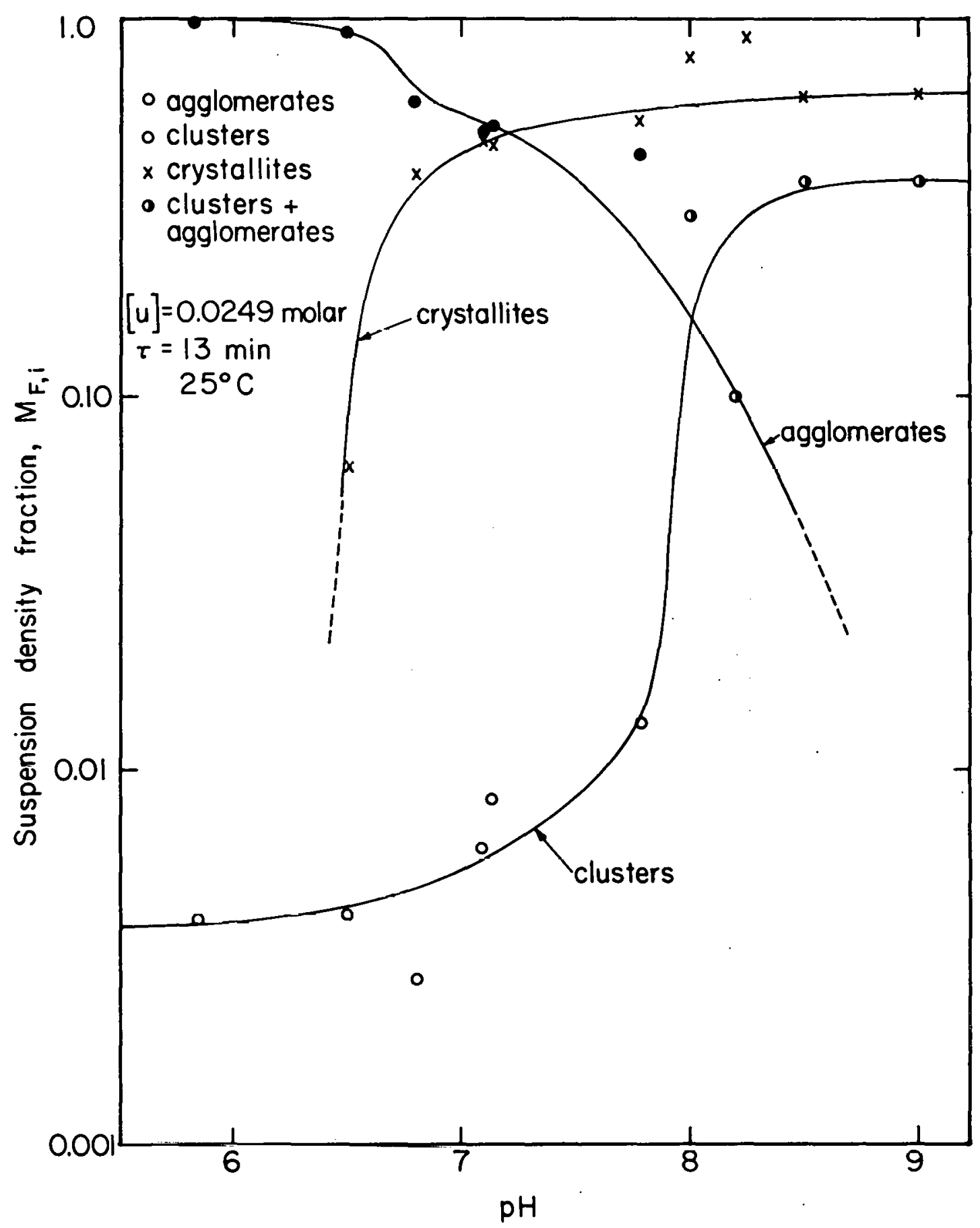

Figure 34. Suspension density fraction as a function of RFIR at a uranium concentration of 0.0249 . 


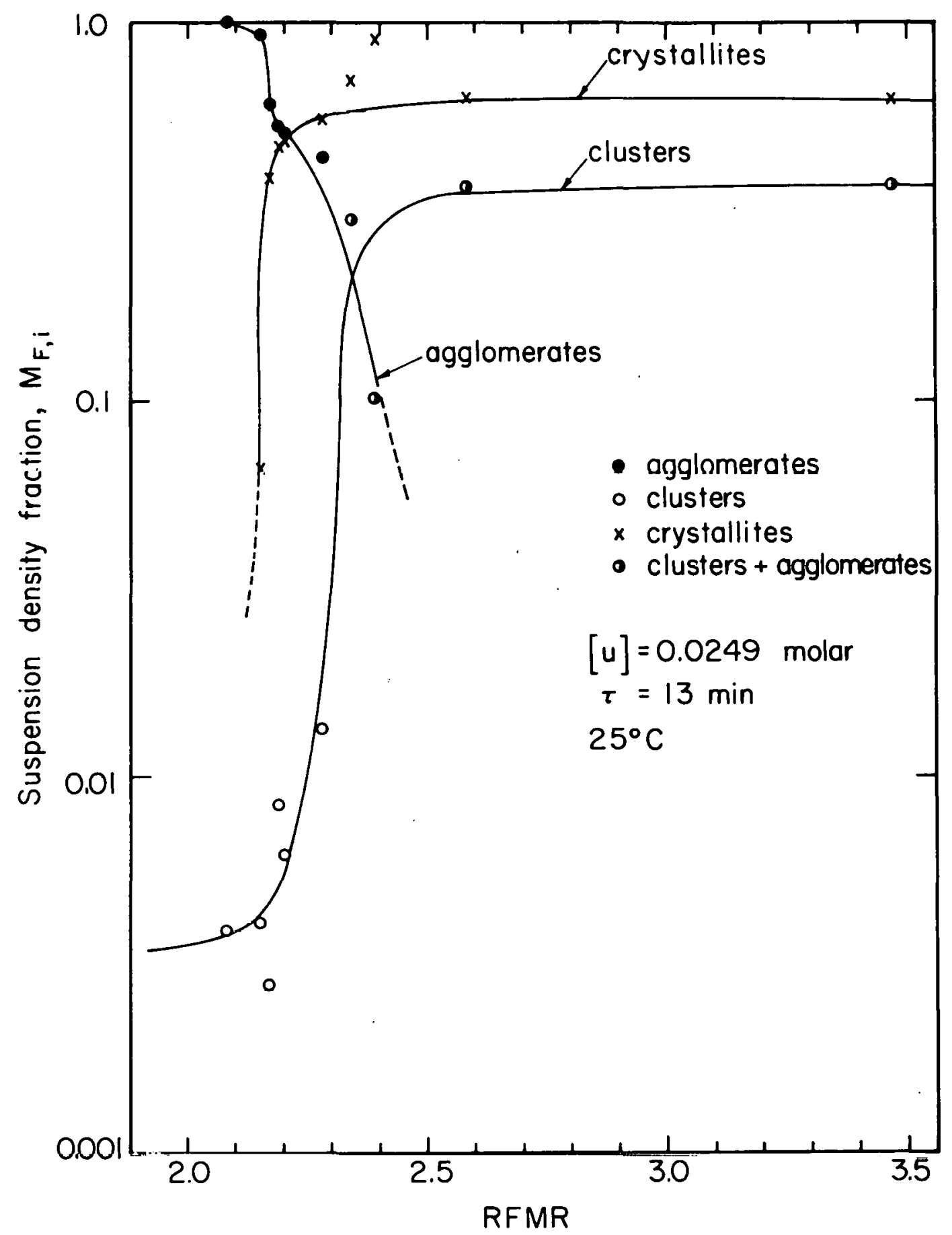

Figure 35. Suspension density Eraction as a function of RFIR at a uranium concentration of 0.0249 . 
By comparing Figures 32 and 33 with Figures 27 and 28 it is seen that the minimum crystallite growth rate occurs at the $\mathrm{pH}$ or RFMR value where the cluster and agglomerate suspension density curves cross each other, whereas the maximum in the agglomerate growth rate occurs at the point where the crystallite and cluster suspension density curves cross. The physical significance of this correlation has not yet been determined.

In attempting to produce a ceramic product with a specific microstructure, once the desired characteristics of the starting powder have been determined a plot such as Figure 32 or 33 can be used in conjunction with the particle size distributions from Equations (9), (13) and (18) to determine the reacting conditions ( $\mathrm{pH}$ or RFMR) which will produce the desired mix of particle sizes.

\section{Phase Analysis}

The X-ray powder patterns of the APU samples were compared with the results reported by Debets and Loopstra (12). Compound III $\left(2 \mathrm{UO}_{3} \cdot \mathrm{NH}_{3} \cdot 3 \mathrm{H}_{2} \mathrm{O}\right)$ in their paper appears to be the dominant phase present in all of the samples prepared at a uranium concentration of 0.0249 molar (runs number 1 to 14); however, a trace of another, unidentified phase is also present in these samples. 
The samples prepared at a constant RFMR and varying uranium concentration (runs 18 to 20 ) show a much greater variation in phases present. The powder pattern shows sample from run number 20 to contain compound III and no detectable amount of any other phase. Sample 18 contains compound IV $\left(3 \mathrm{UO}_{3} \cdot 2 \mathrm{NH}_{3} \cdot 4 \mathrm{H}_{2} \mathrm{O}\right)$ and a significant amount of at least one other unidentified phase. Sample 19 does not contain any of the four phases reported by Debets and Loopstra.

In the pH range abuve 7 il is no surprise that compound III is the dominant phase. However, the lack of compound II $\left(3 \mathrm{UO}_{3} \cdot \mathrm{NH}_{3} \cdot 5 \mathrm{H}_{2} \mathrm{O}\right)$, even in the $\mathrm{pH}$ range below 7, and the presents of phases other that the four reported is not in agreement with the results of Cordfunke. This suggests that the nonequilibrium conditions of the reaction and the nature of the starting material have an effect on the phases present in the $\mathrm{NH}_{3}-\mathrm{UO}_{3}-\mathrm{H}_{2} \mathrm{O}$ system. The results also indicate that the uranium concentration has a much greater effect on the phases present in the system than does the $\mathrm{NH}_{3}: \mathrm{U}$ reacting feed mole ratio. 


\section{SUMMARY AND CONCLUSIONS}

Ammonium polyuranate powders produced in a continuous mixed-suspension, mixed-product-removal precipitator were examined with the scanning electron microscope and the transmission electron microscope and characterized according to particle size distribution, partical shape, and particle morphology.

The SEM studies showed the powders to be composed of three distinct types of particles -- elementary crystallites, clusters of the crystallites and agglomerated clusters. These three types of particles were most distinguishable in the powders produced at the lower values of reacting feed mole ratio. As the RFMR was increased with fixed uranium concentration the crystallites and agglomerates became much more loosely coagulated until at the higher RFMR values the powder samples had no detectable coagulated structure. Variation in the uranium concentration at constant RFMR seems to have a strong effect on cluster and agglomerate sizes but little effect on the particle morphology.

Measurements of the cluster and agglomerate size distributions from the SEM micrographs showed close agreement with the size distributions measured with the coulter counter, showing that the particles observed with the SEM 
are the clusters and agglomerates postulated from the coulter counter results.

The elementary crystallites were characterized with the TEM according to particle size distribution, giving an effective crystallite growth rate and volumetric shape factor. The crystallite growth rate was seen to go through a minimum with respect to $\mathrm{pH}$ and RFMR. This minimum can be explained by the opposing effects on supersaturation with increasing $\mathrm{pH}$ of increasing surface area and decreasing sulubility.

The experimental values obtained for the crystallite growth rates completed the information necessary to apply the kinetic theory of precipitation to the APU system. Values for the important kinetic parameters were calculated and reported. A correlation of the mass fraction of crystallites, clusters, and agglomerates to the reacting $\mathrm{pH}$ and $\mathrm{NH}_{3}: \mathrm{U}$ reacting feed mole ratio was then developed.

It was shown that the surface area and suspension density of the clusters and agglomerates for an APU powder sample can be accurately determined without a value for the crystallite growth rate by assuming the minimum particle sizes to be zero. Further work on other types of precipitates will determine whether or not such a result can be generalized. If so, the number of experimental 
measurements -- necessary for predicting the general characteristics of a powder precipitate would be significantly reduced.

A phase analysis by $\mathrm{X}$-ray diffraction showed the uranium concentration to have the dominant effect in determining the phases present in the precipitate. The nonequilibrium condition of the continuous precipitation also seemed to have an effect on the phases produced. The results of this work show that the particle size distribution, particle structurc, and partiolc oompocition of an inorganic powder can be controlled by proper regulation of the precipitation conditions, and the precipitation process can be described by equations resulting from application of population balances. This implies that the desired microstructure of a final ceramic product can be arbitrarily set and, in conjunction with sintering conditions, the powder properties of the starting material can be systematically controlled to produce the desired product. 


\section{BIBLIOGRAPHY}

1. Adwick, A. G., and R. J. Warmer. 1966. The influence of particle size distributions on the sintering of ceramic powders. Pages 329-343 in the Proceedings of the Particle Size Analysis Conference. The Society for Analytical Chemistry, London, England.

2. Ainscough, J. B., and B. W. Oldfield. 1962. Effect of ammonium diuranate precipitation and conditions on the characteristics and sintering behavior of. uranium dioxide. J. Appl. Chem. 12:418.

3. Amelinckx, S., R. Gevers, and G. Remant. 1970. Modern diffraction and imaging techniques in material sciences. American Elsevier Publishing Company, Inc., New York.

4. Arendt, R. H., R. J. Charles, C. Greskovich, and J.A. Palm. 1973. The role of powder processing in developing material properties. General Electric, Metallurgy and Ceramics Laboratory (Schenectady, New York) Report No. 73CRD330.

5. Behringer, A. J., D. F. Alliet, and R. C. Ackerman. 1970. The correlation of electron microscope results with those of the coulter counter and of surface area in the characterization of polymeric materials. Pages 366-380 in the Proceedings of the Particle Size Analysis Conference. The Society for Analytical Chemistry, London, England.

6. Burke, J. E., and J. H. Rosolowski. 1973. Sintering. General Electric, Metallurgy and Ceramics Laboratory (Schenectady, New York) REport No. 73CRD268.

7. Chalder, G. H., N. F. H. Bright, D. L. Peterson, and L. C. Watson. 1958. The fabrication and properties of uranium dioxide fuel. Proceedings of the second United Nations International Conference on the Peaceful Uses of Atomic Energy 6:590-604. United Nations, Geneva.

8. Clayton, J. C., and S. Aronson. 1961. Some preparative methods and physical characteristics of uranium dioxide powders. J. Chem. and Eng. Data 6:43-51. 
9. Cordfunke, E. H. P. 1962. On the uranates of ammonium$I$, the ternary system $\mathrm{NH}_{3}-\mathrm{UO}_{3}-\mathrm{H}_{2} \mathrm{O}$ ). J. Inorg. Nucl. Chem. $24: 303$.

10. Crowl, V. T. 1966. Particle size analysis by counting from electron micrographs. Pages 36-44 in the Proceedings of the Particle Size Analysis Conference. The Society for Analytical Chemistry, London, England.

11. Cullity, B. D. 1956. Elements of X-ray Diffraction. Addison-Wesley Publishing Company, Reading, Massachusetts.

12. Debets, P. C., and B. O. Loopstra. 1963. On the uranates of ammonia-II, X-ray investigation of the compounds in the system $\mathrm{NH}_{3}-\mathrm{UO}_{3}-\mathrm{H}_{2}$ ). J. Inorg. Nucl. Chem. $25: 945$.

13. Deptula, A. 1962. A study of composition of ammonium uranates. Nukleonika 7:265-275.

14. Doi, H., and T. Ito. 1964. Significance of physical state of starting precipitate in growth of uranium dioxide particles. J. Nucl. Mat. 11(1):94-106.

15. Ewing, R. A., S. J. Kiehl, Jr., and A. E. Bearse. 1956. Investigation of ammonium uranates. BMI-1115.

16. Garside, J., and S. J. Jancic. 1976. Prediction and measurement of crystal size distributions for sizedependent growth. Unpublished paper presented at the 69th AIChE Annual Meeting, Chicago, Illinois.

17. Goldstein, J. I., and H. Yakowitz. 1975. Practical scanning electron microscopy. Plenum Press, New York.

18. Hoyt, R. D. 1978. Precipitation Kinetics of a continuous precipitator, with application to the precipitation of ammonium polyuranate. $\mathrm{Ph} . \mathrm{D}$. Thesis. Iowa State University, Ames, Iowa.

19. Janov, J., P. G. Alfredson, and V. K. Vilkaitis. 1971. The influence of precipitation conditions on the properties of ammonium diuranate and uranium dioxide powders. AAEC/E220. 
20. Johari O., and S. Bhattacharyya. 1969. The application of scanning electron microscopy for the characterization of powders. Powder Technol, $2(1968 / 69): 335-348$.

21. Johari, O., and P. B. DeNee. 1972. Handling, mounting and examination of particles for scanning electron microscopy. Proceedings of the Fifth Annual Scanning Electron Microscope Symposium 1:249-256. IIT Research Institute, Chicago, IIlinois.

22. Nielsen, A. E. 1964. Kinetics of precipitation. The Macmillan company, New York.

23. Orr, C., Jr. 1966. Particulate technology. The Macmillan Company, New York.

24. Palei, P. N. 1970. Analytical chemistry of uranium. Ann Arbor-Humphrey Science Publishers, Ann Arbor.

25. Randolph, A. D., and M. A. Larson. 1971. Theory of particulate processes. Academic Press, New York. $251 \mathrm{pp}$.

26. Robertson, J.A. L. 1975. Nuclear fuel failures, their causes and remedies. Pages 2-2 to 2-14 in the Proceedings of Joint Topical Meeting on Commercial Nuclear Fuel Technology Today, April 28-30, 1975. ANS and CNA, Toronto, Canada.

27. Rosauer, E. A., and C. B. Wagner. 1953. Vacuum evaporation of metals for shadow casting. J. Appl. phy. $24: 1401$.

28. Runnalls, O. J. C. 1959. Uranium dioxide fuel elements. Pages 228-266 in H. H. Hausner, ed. Nuclear Fuel Elements. Reinhold Publishing Corporation, New York.

29. Stevenson, D. G. 1964. Theoretical aspects of the design and operation of continuous chemical precipitators. Trans. Instn. Chemical Engrs. 42:T316T342.

30. Sutton, J. 1949. The hydrolysis of the uranyl ion, Part I. J. Chem. Soc., Suppl. Iss .:275=286. 
31. White, E. W. 1968. Applications of the SEM to ceramic studies. Proceedings of the Symposium on, The Scanning Electron Microscope -- The Instrument and its Applications 89-94. IIT Research Institute, Chicago, Illinois.

32. Wyckoff, R. W. G. 1949. Electron microscopy, technique and applications. Interscience Publishers, New York. 


\section{ACKNOWLEDGMENTS}

I would like to thank Dr. L. E. Burkhart for his words of advice and encouragement during the course of this work.

Appreciation is also expressed to Max Petersen for the hours he spent counting particles, and to Harlen Baker and Jerry Amenson for their assistance in operating the electron microscopes.

Finally, I would like to acknowledge Mike Murtha for being a consistent and reliable source of information in all phases of my work. 